\title{
Intergrated Metagenomics and Metabolomics Analysis Discovers Nematicidal Microbes, Enzymes and Metabolites From the Plant Rhizosphere Microbiota
}

\section{Xiuyun Zhao}

Huazhong Agricultural University: Huazhong Agriculture University

Changchun Lin

Huazhong Agricultural University: Huazhong Agriculture University

Wenfang Yuan

Huazhong Agricultural University: Huazhong Agriculture University

\section{Song Ruan}

Huazhong Agricultural University: Huazhong Agriculture University

Gaofu Qi ( $\square$ qigaofu@mail.hzau.edu.cn )

Huazhong agricultural university https://orcid.org/0000-0002-6396-2819

\section{Research}

Keywords: Meloidogyne incognita, rhizophere microbiota, metagenomics analysis, metabolomics analysis, acetophenone, Bacillus amyloliquefaciens

Posted Date: February 12th, 2021

DOI: https://doi.org/10.21203/rs.3.rs-187899/v1

License: (c) (1) This work is licensed under a Creative Commons Attribution 4.0 International License.

Read Full License 
Intergrated metagenomics and metabolomics analysis discovers nematicidal microbes, enzymes and metabolites from the plant rhizosphere microbiota

Xiuyun Zhao, Changchun Lin, Wenfang Yuan, Song Ruan, Gaofu Qi†

Xiuyun Zhao: xiuyunzh@mail.hzau.edu.cn; Changchun Lin:1966703629@qq.com; Wenfang Yuan:1663503327@qq.com; Song Ruan: 476478609@qq.com

†Corresponding author. Email: qigaofu@mail.hzau.edu.cn

College of Life Science and Technology, Huazhong Agricultural University, Wuhan 430070,

PR China 


\begin{abstract}
Background: Root-knot nematode Meloidogyne incognita infects root systems of many crops resulting in huge decrease of crop production. Nematicidal microorganisms provides a safe and effective strategy to control $M$. incognita infection. In order to find more microorganisms with high activity and new nematicidal metabolites, we collected the $M$. incognita infected tobacco rhizosphere soils (RNI) and non-infected tobacco rhizosphere soils (NS), and investigated their microbial community and network via metagenomics and metabolomics analysis.
\end{abstract}

Results: Microbial networks of RNI soils were very different from the NS soils. Many nematicidal microorganisms were enriched in the NS soils, including some isolates such as Aspergillus, Achromobacter, Acinetobacter, Bacillus, Burkholderia, Comamonas, Enterobacter, Lysobacter, Microbacterium, Paenibacillus, Pantoea, Pseudomonas, Streptomyces and Variovorax. Enzymes analysis showed these nematicidal microorganisms can produce proteases, chitinase and lipases. The functions genes belonging to pathways of secondary metabolites biosynthesis and carbohydrate transport and metabolism were overrepresented in the rhizophere microbiota of NS soils comparing with the RNI soils. 102 metabolites contents were significantly different between the RNI and NS rhizosphere microbiota. 35 metabolites were overrepresented in the NS soils comparing the RNI samples, including acetophenone. Acetophenone showed high nematicidal $\left(\mathrm{LC}_{50}=0.66 \mu \mathrm{g} / \mathrm{ml}\right)$ and avoidance activity against M. incognita. A isolate of Bacillus amyloliquefaciens W1 with production of acetophenone can kill $98.8 \%$ of $M$. incognita.

Conclusions: In general, the rhizophere microbiota of NS soils could produce volatile materials, multiple enzymes and secondary metabolites against nematode. Collectively, the microbiota of NS and RNI rhizophere differed significantly in microbial network structure, community composition, function genes and metabolites. Collectively, combination of multi-omics analysis and culture-dependent technology is powerful for finding nematicidal microorganisms and metabolites from soil.

Keywords: Meloidogyne incognita; rhizophere microbiota; metagenomics analysis; metabolomics analysis; acetophenone; Bacillus amyloliquefaciens

\title{
Background
}


The root-knot nematode Meloidogyne incognita can parasitize plant roots and causes dramatic yield losses in many different crops worldwide [1], but the management options available for effectively controlling root-knot nematode are extremely limited. Although traditional control methods such as crop rotation, disease resistance breeding and use of chemical pesticides have reduced nematode diseases indeed, the crop yield losses caused by $M$. incognita are still enormous. Fortunately, some microbial agents can effectively control root-knot nematodes to reduce M. incognita infection. These microorganisms include rhizosphere bacteria, nematophagous fungi, specialized parasitic bacteria, actinomycetes, etc, [2, 3]. Among them, rhizosphere microbiota are especially interested, and currently, there is a growing interest in studying rhizosphere microbiota in the root-knot nematode infected soils [4].

Many rhizosphere microorganisms have been isolated and selected as biological agents for controlling root-knot nematodes [5-7]. However, application of microbial agents also raised a novel problem that is the control effect of microbial agents are often unstable in the field [8]. One of the main reasons is that these beneficial microorganisms can't stably colonize in soil and plant rhizosphere for a long time due to their disability to adapt to complex soil environment and climate conditions [9]. In particular, many farmland soils in the Southern China are seriously degraded and the soil ecological environment is deteriorating year by year, resulting in "bad" soil microbial communities and unbalanced networks. Thus, the colonization of beneficial microorganisms will be very difficult in these soils $[10,11]$.

Generally, researchers often pay more attentions to screening of microbes for biocontrol of root-knot nematodes, but the interaction relationships among root-knot nematodes, rhizophere microbiota and biocontrol microbes are rarely understood in the soil ecosystem. Nowadays, how to control the soil micro-ecological environment and make the beneficial microorganisms colonize more stable and exert their effects furthest in soils is one of the key points to improve their biocontrol effects. Investigation of microbial interactions may help to resolve this bottle-neck problem. Microbial interactions encompass a spectrum ranging from antagonistic and competition (resource competition) to cooperative, syntrophic and mutualistic interactions. Network analysis is favorable for exploring the organisation and dynamics of microbial interactions and niches [12.13], and help us to find "good friends" (e.g. nematicidal microorganisms) and improve their colonization efficiency in soils. 
Metagenomics and metabolomics analysis have enabled the study of microbial ecosystem structure to a greater depth and accuracy. Metagenomics sequencing technology is an interesting tool for selection of the desirable biocontrol microbes [14], and metabolomics is favorable for finding active metabolites produced by microorganisms [15]. The main object of this project is to study the important functional genes and key microorganisms related to the occurrence of root-knot nematode disease, and screen novel nematicidal microorganisms and metabolites against root-knot nematodes based on the metagenomic and metabolomic analysis. This study will provide a way for effective control of root-knot nematodes by improving soil micro-ecology, promoting the colonization and efficiency of beneficial microorganisms, and developing new microbial metabolites with anti-nematode activity.

\section{Methods}

\section{Soil sampling and properties analysis}

Fifteen root-knot nematode $M$. incognita infected tobacco fields (RNI) and fifteen non-infected tobacco fields (NS) were located in Enshi City, Hubei Province, China $\left(29^{\circ} 22^{\prime}-29^{\circ} 58^{\prime} \mathrm{N}, 109^{\circ} 20^{\prime}-109^{\circ} 22^{\prime} \mathrm{E}\right)$, with elevations ranging from 990 to $1,127 \mathrm{~m}$ and subtropical humid climate. The soil is yellow-brown soil (classified as Alfisols). Rhizosphere soil samples were collected from ten tobacco plants in each field $\left(667 \mathrm{~m}^{2}\right)$ in August 2018 at the maturation stage of tobacco. The tobaccos were removed gently from the field, then the loosely adherent soils were removed by vigorous shaking, and the soils adhering to root were collected with a brush. The collected rhizosphere soil of ten plants in each field was mixed together as a composite soil sample. Soil samples were sieved (2-mm mesh) to remove plant roots, debris and stones. Each soil sample was divided into two parts: one was stored at $-80{ }^{\circ} \mathrm{C}$ for DNA and metabolites extraction within one week, and the other one was stored at $4{ }^{\circ} \mathrm{C}$ for soil properties and root-knot nematode analysis. Quantification of M. incognita in soil samples was determined via real-time quantitative PCR [16].

Soil organic matter (SOM) content was assayed by the acidified potassium dichromate $\left(\mathrm{K}_{2} \mathrm{Cr}_{2} \mathrm{O}_{7}-\mathrm{H}_{2} \mathrm{SO}_{4}\right)$ heating method [17]. Alkali-hydrolyzable nitrogen (AN) content was determined by alkaline hydrolysis diffusion method. Available phosphorus (AP) and potassium (AK) contents were determined photometrically by a flame spectrophotometer. After suspending with deionized water (soil $:$ water $=1: 2.5, \mathrm{w} / \mathrm{v}$ ), soil $\mathrm{pH}$ was determined 
using a $\mathrm{pH}$ meter. Urease activity was detected by colorimetric determination of ammonium. Catalase activity was determined by colorimetric assay using $\mathrm{K}_{2} \mathrm{Cr}_{2} \mathrm{O}_{7} /$ acetic acid reagent. Invertase activity was measured by 3,5-dinitrosalicylic acid colorimetry with sucrose as substrate. Phosphatase activity was measured by $p$-nitrophenol colorimetry [18]. Soil water content (SWC), capillary moisture capacity (SCMC), bulk density (BD), total porosity (STP), capillary porosity (SCP), aeration porosity (SAP) and mean weight diameter (MWD) were routinely measured according to previous methods $[17,19]$.

\section{Metagenomic sequencing data pretreatment and metagenome assembly}

For each soil sample, DNA was extracted from $1.0 \mathrm{~g}$ soils using the FastDNA Spin Kit (MP Biomedicals, USA). DNA concentration was measured using Qubit® dsDNA Assay Kit in Qubit ${ }^{\circledR}$ 2.0 Flurometer (Life Technologies, CA, USA). $1 \mu \mathrm{g}$ DNA per sample was used to construct library. Sequencing libraries were generated using NEBNext ${ }^{\circledR}$ Ultra ${ }^{\mathrm{TM}}$ DNA Library Prep Kit for Illumina (NEB, USA) and index codes were added to attribute sequences to each sample. Briefly, the DNA sample was fragmented by sonication to a size of $350 \mathrm{bp}$, then DNA fragments were end-polished, A-tailed, and ligated with the full-length adaptor for PCR amplification. PCR products were purified by AMPure XP system and libraries were analyzed for size distribution by Agilent2100 Bioanalyzer and quantified using real-time PCR. Clustering of the index-coded samples was performed on a cBot Cluster Generation System according to the manufacturer's instructions. After cluster generation, the library preparations were sequenced on an Illumina HiSeq platform and paired-end reads were generated.

Preprocessing the raw data using Readfq V8 was conducted to acquire the clean data for subsequent analysis. The reads that are of host origin were filtered using Bowtie2.2.4 software. The clean data was assembled and analyzed by MEGAHIT software (v1.0.4-beta), then interrupted the assembled Scaftigs from N connection and leave the Scaftigs without N [20]. All samples' clean data were compared to each Scaffolds respectively by Bowtie2.2.4 software to acquire the PE reads not used. All the reads not used in the forward step of all samples were combined and then used the software SOAPdenovo V2.04/MEGAHIT v1.0.4-beta for mixed assembly, broke the mixed assembled Scaffolds from $\mathrm{N}$ connection and obtained the Scaftigs, then filtered the fragment shorter than $500 \mathrm{bp}$ in all of Scaftigs for 
statistical analysis.

\section{Gene prediction and abundance analysis}

The Scaftigs ( $\geq 500 \mathrm{bp}$ ) assembled from both single and mixed assembly were all predicted the ORF by MetaGeneMark V2.10 software. For ORF prediction, the CD-HIT V4.5.8 software was adopted to redundancy and obtained the unique initial gene catalogue [21]. The clean data of each sample was mapped to initial gene catalogue using Bowtie2.2.4 and get the number of reads to which genes mapped in each sample. We filtered the gene with the number of reads $\leq 2$ in each sample and obtained the gene catalogue Unigenes eventually used for subsequently analysis. Based on the number of mapped reads and the length of gene, the abundance information of each gene in each sample was counted.

\section{Taxonomy prediction}

DIAMOND V0.9.9 software was used to blast the Unigenes to the sequences of bacteria, fungi, archaea and viruses which are all extracted from the NR database of NCBI [22]. The number of genes and the abundance information of each sample in each taxonomy hierarchy (kingdom, phylum, class, order, family, genus, specie) were obtained. The abundance of a specie in one sample equal the sum of the gene abundance annotated for the specie. The gene number of a specie in a sample equal the number of genes whose abundance are nonzero.

Principal component analysis (PCA) and non-metric multi-dimensional scaling (NMDS) analysis were performed based on the abundance of each taxonomic hierarchy. The difference between two groups was tested by Anosim analysis. Metastats and LEfSe analysis were used to find the different species between groups. Permutation test between groups was used in Metastats analysis for each taxonomy and get the P value, then used Benjamini and Hochberg False Discovery Rate to correct $\mathrm{P}$ value and acquired q value [23]. LEfSe analysis was conducted by LEfSe software (the default LDA score is 3). Finally, random forest (RandoForest) was used to construct a random forest model, screen out important species by MeanDecreaseAccuracy and MeanDecreaseGin, then cross-validate each model and plot the ROC curve.

\section{Functional database annotations}

We adopted DIAMOND V0.9.9 software to blast Unigenes to functional database KEGG, 
eggNOG, and CAZy database. For each sequence's blast result, the best Blast Hit was used for subsequent analysis. The relative abundance of different functional hierarchy was counted. The gene number of each sample in each taxonomy hierarchy was obtained. Based on the abundance of each taxonomy hierarchy, not only the counting of annotated gene numbers, the exhibition of the general relative abundance situation, the exhibition of abundance cluster heat map, and the decrease-dimension analysis of PCA and NMDS were conducted, but also the Anosim analysis of the difference between groups based on functional abundance, comparative analysis of metabolic pathways, the Metatat and LEfSe analysis of functional difference between groups were performed.

\section{Network construction and analysis}

The relative abundances of species or functional genes were used to construct microbial or functional networks using the Molecular Ecological Network Analysis Pipeline (MENAP), respectively. Briefly, species or gene abundances were normalized to the standardized relative abundances (SRA) [12]. A matrix of species SRA or genes SRA, a matrix of soil variables, and a species or genes annotation file were prepared. The SRA matrix was submitted to MENAP to construct the NS and RNI microbial or functional networks, respectively [24, 25]. Modules were detected by the greedy modularity optimization. Three files were generated for network graph visualization by Cytoscape 3.7.2 software. The network graph was represented using different species or genes (nodes) with positive or negative interactions (edges). Positive interactions indicate the abundances of these species or genes changed following the same trend across different soil samples. Negative interactions indicate the abundances of those species or genes changed following the opposite trend in different soil samples [26, 27].

The topological role of each node (specie) was defined by two parameters: within-module connectivity $(Z i)$ and among-module connectivity $(P i)$. $Z i$ value described how well a node was connected to other nodes within its own module. $P i$ value described how well a node was connected to different modules [28]. Threshold values of $Z i$ and $P i$ for categorizing nodes were 2.5 and 0.62 , respectively. According to the threshold values of $Z i$ and $P i$, the nodes were divided into four different categories. (i) Peripheral nodes (named specialists) with low $Z i(<2.5)$ and low $P i(<0.62)$ values. These nodes have only a few links and almost always 
are connected to the nodes within their own modules. (ii) Connectors (named generalists) have low $Z i$ values $(<2.5)$ but high $P i$ values $(>0.62)$. They are highly connected with other modules. (iii) Module hubs (also named generalists) with high $Z i$ values (>2.5) but low $P i$ values $(<0.62)$, are highly connected with many nodes in their own modules. (iv) Network hubs (named super-generalists) have both high $Z i(>2.5)$ and $P i(>0.62)$ values. Generalists (connectors, module hubs) and network hubs are the keystone microorganisms, which are critical in maintaining the network stability and microbial community structure and function [13].

\section{Untargeted metabolomics analysis}

Metabolites of five NS samples and five RNI samples were measured by untargeted metabolomics analysis. For each soil sample, $100 \mathrm{mg}$ soils were grounded with liquid nitrogen and the homogenate was suspended with chilled methanol and $0.1 \%$ formic acid. The samples were incubated on ice for $5 \mathrm{~min}$ and then were centrifuged at $15,000 \mathrm{~g}$ and $4{ }^{\circ} \mathrm{C}$ for 5 min. The supernatant was diluted to final concentration containing $60 \%$ methanol by LC-MS grade water. The samples were filtrated with $0.22 \mu \mathrm{m}$ filter and then were centrifuged at $15,000 \mathrm{~g}$ and $4{ }^{\circ} \mathrm{C}$ for $10 \mathrm{~min}$. Finally, the filtrate was injected into the LC-MS/MS system. LC-MS/MS analyses were performed using a Vanquish UHPLC system (Thermo Fisher) coupled with an Orbitrap Q Exactive HF-X mass spectrometer (Thermo Fisher). Samples were injected onto an Hyperil Gold column $(100 \times 2.1 \mathrm{~mm}, 1.9 \mu \mathrm{m})$ at a flow rate of 0.2 $\mathrm{ml} / \mathrm{min}$. The eluents for the positive polarity mode were eluent A1 (0.1\% formic acid in water) and eluent B (methanol). The eluents for the negative polarity mode were eluent A2 (5 mM ammonium acetate, $\mathrm{pH}$ 9.0) and eluent B (methanol). The solvent gradient was set as follows: 2\% B, 1.5 min; 2-100\% B, $12.0 \mathrm{~min} ; 100 \%$ B, 14.0 min; 100-2\% B, $14.1 \mathrm{~min} ; 2 \% \mathrm{~B}, 16 \mathrm{~min}$. Q Exactive HF-X mass spectrometer was operated in positive/negative polarity mode with spray voltage of $3.2 \mathrm{kV}$, capillary temperature of $320^{\circ} \mathrm{C}$, sheath gas flow rate of 35 arb and aux gas flow rate of 10 arb. The raw data generated by UHPLC-MS/MS were processed using the Compound Discoverer 3.0 (CD 3.0, Thermo Fisher) to perform peak alignment, peak picking, and quantitation for each metabolite. Peak intensities were normalized to the total spectral intensity. The normalized data were used to predict the molecular formula based on 
additive ions, molecular ion peaks and fragment ions, and then peaks were matched with the mzCloud and ChemSpider databases to obtain the accurate qualitative and relative quantitative results. These metabolites were annotated using the KEGG, HMDB, and Lipidmaps databases $[29,30]$.

Principal components analysis (PCA) and partial least squares discriminant analysis (PLS-DA) were performed at metaX. Univariate analysis ( $t$-test) was applied to calculate the statistical significance ( $P$-value). The metabolites with VIP $>1$ and $P$-value $<0.05$ and fold change $\geq 2$ or FC $\leq 0.5$ were considered to be differential metabolites. Volcano plots were used to filter metabolites of interest which based on $\log 2(\mathrm{FC})$ and $-\log 10(\mathrm{P}$-value) of metabolites. For clustering heat maps, the data were normalized using z-scores of the intensity areas of differential metabolites and were plotted by Pheatmap package in R language. The functions of these metabolites and metabolic pathways were studied using the KEGG database.

Bacillus amyloliquefaciens W1 culture was analyzed by untargeted metabolomics. W1 was incubated in $100 \mathrm{ml}$ of $\mathrm{M} 9$ medium $\left(\mathrm{MgSO}_{4} \cdot 7 \mathrm{H}_{2} \mathrm{O} 0.492 \mathrm{~g}, \mathrm{CaCl}_{2} 0.011 \mathrm{~g}\right.$, $\mathrm{Na}_{2} \mathrm{HPO}_{4} \cdot 12 \mathrm{H}_{2} \mathrm{O} 17 \mathrm{~g}, \mathrm{KH}_{2} \mathrm{PO}_{4} 3 \mathrm{~g}, \mathrm{NaCl} 0.5 \mathrm{~g}, \mathrm{NH}_{4} \mathrm{Cl} 1 \mathrm{~g}$, glucose $4 \mathrm{~g}, 1000 \mathrm{ml} \mathrm{ddH}{ }_{2} \mathrm{O}$ ) at $37{ }^{\circ} \mathrm{C}$ and $180 \mathrm{rpm}$ for $48 \mathrm{~h}$. The culture was centrifuged at 8,000 g for $15 \mathrm{~min}$, and the supernatant was collected and mixed with equal volume of ethyl acetate, then centrifuged at $8,000 \mathrm{~g}$ for $15 \mathrm{~min}$. The extracted liquid was dried by rotary evaporation at $65{ }^{\circ} \mathrm{C}$, then dissolved in $2 \mathrm{ml}$ ethanol following with filtration using filter membrane. $100 \mu \mathrm{l}$ of sample was mixed with $400 \mu \mathrm{l}$ of $80 \%$ methanol and $0.1 \%$ formic acid by well vortexing, incubated on ice for $5 \mathrm{~min}$, then centrifuged at $15,000 \mathrm{~g}$ and $4{ }^{\circ} \mathrm{C}$ for $10 \mathrm{~min}$. The supernatant was diluted to final concentration containing $53 \%$ methanol by LC-MS grade water. After centrifugation at $15,000 \mathrm{~g}$ and $4{ }^{\circ} \mathrm{C}$ for $10 \mathrm{~min}$, the supernatant was collected for detection by the LC-MS/MS system and the data analysis was conducted as the above mentioned methods.

\section{Analysis of nematicidal activity of metabolites}

M. incognita was maintained on tomato roots. A lot of galls formed in the roots after infection. Nematode eggs were isolated from galls. Before detecting the nematicidal activity of metabolites and microbes, the galls were peeled off from root and placed in water at $20{ }^{\circ} \mathrm{C}$ until the second-stage juveniles (J2) were hatched. J2 juveniles were used in the following 
nematicidal bioassays.

The nematicidal activity of metabolites were detected as previously described [31]. The nematicidal activities of different concentrations of acetophenone $(0.412,0.459,0.515,0.589$, $0.687,0.824,1.03$, and $2.06 \mu \mathrm{g} / \mathrm{ml}$ ) were tested against $M$. incognita $\mathrm{J} 2$ juveniles for $24 \mathrm{~h}$, respectively. Each concentration of acetophenone was detected with three replicates. The $\mathrm{LC}_{50}$ and $\mathrm{LC}_{90}$ values of acetophenone were calculated. Assay for nematode avoidance of acetophenone was conducted as our previous study [32]. Water-agar medium (1.5\% agar) was prepared in $10-\mathrm{cm}$ plates. Two or four $\mu \mathrm{L}$ acetophenone were dropped on one side of the plate, and 2 or $4 \mu \mathrm{L} \mathrm{ddH}_{2} \mathrm{O}$ were dropped on the other side as a control. Approximately 50 larvae of M. incognita were transferred to the middle of plate. After $2 \mathrm{~h}$, the nematodes in each section were counted and avoidance index was calculated.

\section{Isolating nematicidal microorganisms from rhizospheric soils}

Ten grams of the NS rhizospheric soil was mixed with $90 \mathrm{ml}$ of sterile water, then used for spreading TSB agar plates (tryptone $1.5 \%$, soy peptone $0.5 \%, \mathrm{NaCl} 0.5 \%$, and agar $1.5 \%$, $\mathrm{pH} 7.2$ ) for isolating bacteria, and PDA plates (potato $20 \%$, glucose $2 \%$, agar $1.5 \%$ ) for isolating fungi after serial dilution with sterile water. After incubation, the bacteria and fungi with different morphology were isolated from plates for screening the ones with nematicidal activity

For nematicidal activity assay, the isolated bacteria were incubated in LB medium at $37{ }^{\circ} \mathrm{C}$ and $200 \mathrm{rpm}$ for $48 \mathrm{~h}$, and the isolated fungi were incubated in PD medium at $28{ }^{\circ} \mathrm{C}$ and $200 \mathrm{rpm}$ for 5 days. After incubation, the broth was centrifuged at 8,000 $\mathrm{g}$ for $5 \mathrm{~min}$ for collecting supernatant. $200 \mu \mathrm{l}$ of supernatant and $\sim 50$ numbers of $M$. incognita $\mathrm{J} 2$ juveniles were mixed together and then added to each well in 96-well plates. After incubation at $20{ }^{\circ} \mathrm{C}$ for $24 \mathrm{~h}$, the mortality of nematode was counted. The nematodes without detectable movement were judged as dead. Tests were done with three replicates. LB or PD medium was added in the assay as control [31].

Nematicidal bacterial and fungal isolates were identified based on 16S rRNA or $18 \mathrm{~S}$ rRNA genes sequence. Briefly, the bacterial or fungal genomic DNA was extracted using the DNA isolation kit (TIANGEN), then the bacterial 16S rRNA gene or fungal 18S rRNA gene 
was amplified, respectively $[33,34]$. Sequence alignment was performed with Clustal $\mathrm{W}$, and the phylogenetic tree was constructed using the software MrBayes 3.27, then visualized with iTOL $[35,36]$.

The enzymes activity including chitinase, protease and lipase were detected in the isolated nematicidal bacteria and fungi, respectively. Protease activity was detected using the clearing zones method [37]. Single bacterial or fungal colony was incubated on the surface of selection medium (nonfat dried skimmed milk $5 \%$, agar $2 \%$ ) plates at $28{ }^{\circ} \mathrm{C}$ for $48 \mathrm{~h}$, then the diameters of clearing zone (D2) and the bacterial or fungal colony (D1) were measured, respectively. The ratio of D2 to D1 was calculated as an indicator of protease activity. Chitinase activity was also detected using the clearing zones method on the surface of chitin medium $\left(\mathrm{NaCl} 1.5 \mathrm{~g}, \mathrm{KH}_{2} \mathrm{PO}_{4} 0.3 \mathrm{~g}, \mathrm{~K}_{2} \mathrm{HPO}_{4} 0.7 \mathrm{~g}, \mathrm{FeSO}_{4} \cdot \mathrm{H}_{2} \mathrm{O} 0.02 \mathrm{~g}, \mathrm{MgSO}_{4} 0.5\right.$ g, agar 20 g, $250 \mathrm{ml}$ of $2 \%$ colloidal chitin, adding $\mathrm{H}_{2} \mathrm{O}$ to $1000 \mathrm{ml}$, pH 7.0). Lipase activity was detected on the surface of selection medium (peptone $1 \%$, yeast powder $0.5 \%, \mathrm{NaCl} 1 \%$, glyceryl triacetate $3 \%$, bromcresol purple $0.004 \%$, agar $2 \%$ ) at $28{ }^{\circ} \mathrm{C}$ for $48 \mathrm{~h}$. Glycerol triacetate produces free fatty acids under the catalysis of lipase, which reduces the $\mathrm{pH}$ of the medium and makes medium change from blue purple to yellow. The diameters of the yellow clearing zone (D2) and the bacterial/fungal colony (D1) were measured, and the ratio of D2 to D1 was calculated as an indicator of lipase activity.

\section{Statistics analysis}

Differences in soil properties, compounds, microbial or gene abundances and network indexes between the NS and RNI soils were compared by a least-significant-difference (LSD) test. Correlations between metabolite and nematode number was analyzed by Pearson correlation analysis with SPSS 20 software [18].

\section{Results}

\section{Soil properties were different between the NS and RNI soils}

It was found that the number of $M$. incognita was very different between the non-infected tobacco rhizosphere soil (NS) and the root-knot nematode infected tobacco rhizosphere soil (RNI). The number of M. incognita in NS was 0.08 to 3.56, with an average value of 0.98 ; whereas the number of M. incognita in RNI was 325.21 to 3070.75 , with an average value of 
1091.76 (table S1). The number of $M$. incognita in RNI was significantly $(P<0.05)$ higher than that in NS.

Soil properties were also different between NS and RNI. The activities of invertase and catalase, $\mathrm{pH}, \mathrm{CEC}, \mathrm{Fe}, \mathrm{Ca}, \mathrm{SWC}, \mathrm{STP}, \mathrm{SCMC}$ and MWD were all significantly $(P<0.05)$ higher in the NS soils compared to the RNI soils. Conversely, the phosphatase activity, AK and $\mathrm{BD}$ were all significantly $(P<0.05)$ higher in the RNI soils than the NS soils. Catalase activity, CEC, Fe, Ca, STP and MWD were significantly $(P<0.05)$ negatively correlated with the number of $M$. incognita, while $\mathrm{AP}$ and $\mathrm{BD}$ were significantly positively correlated with the number of $M$. incognita (table S2).

\section{Taxonomic diversity of rhizosphere microbiota and network structure}

The taxonomic diversity and functional potential of the rhizosphere microbiota were investigated in the tobacco maturation period. After metagenome sequencing and bioinformatics analysis (tables S3 and S4), taxonomic assignment of the microbial cell fraction from the tobacco rhizosphere showed that $85.7 \%, 0.2 \%, 2.1 \%$, and $0.3 \%$ of the sequence reads corresponded to the domains Bacteria, Eukaryota, Archaea and virus, respectively (fig. S1 A). Principal Co-ordinates Analysis showed significant difference (Anosim, $P<0.01$ ) between the microbial community structure and composition of the NS and the RNI rhizosphere microbiota (fig. S2A).

Proteobacteria, Acidobacteria, Actinobacteria and Gemmatimonadetes dominated the bacterial community (fig. S2B). At the class level, top 10 were Alphaproteobacteria, Gammaproteobacteria, Betaproteobacteria, Actinobacteria, Acidobacteriia, Deltaproteobacteria, Ktedonobacteria, Gemmatimonadetes, Chitinophagia and Nitrospira (fig. S2C). At the family level, top 10 were Sphingomonadaceae, Rhodanobacteraceae, Acidobacteriaceae, Gemmatimonadaceae, Bradyrhizobiaceae, Burkholderiaceae, Comamonadaceae, Chitinophagaceae, Ktedonobacteraceae and Streptomycetaceae (fig. S2D). 88 species spanning Achromobacter (two), Acinetobacter (six), Alcaligenes faecalis (one), Bacillus (five), Burkholderia (two), Comamonas (five), Delftia acidovorans (one), Enterobacter (one), Escherichia (one), Lactobacillus brevis (one), Lysobacter (one), Microbacterium (three), Paenibacillus (six), Pantoea (one), Pseudomonas fluorescens (one), 
Pseudomonas (thirty-one), Pseudoxanthomonas (three), Rhizobium (three), Sinorhizobium (one), Streptomyces (six), Variovorax (six), and Virgibacillus (one), all became enriched in the NS soils comparing with the RNI soils (fig. S3). Aspergillus belonging to Ascomycota was also enriched in the NS soils. Relative abundance of these enriched bacteria were positively correlated with the CEC, MWD, Ca and Fe contents, while were negatively correlated with the AK content and the number of M. incognita $(P<0.05)$. We got 62 bacterial isolates and 2 fungal isolates that showed nematicial activity from the NS rhizosphere soils (fig. S4). Among these bacteria, 47 isolates showed a mortality more than $80 \%$ towards $M$. incognita. These isolates belonged to Achromobacter (1), Acinetobacter (4), Aspergillus (2), Bacillus (19), Burkholderia (1), Comamonas (1), Enterobacter (6), Lysobacter (1), Microbacterium (3), Paenibacillus (1), Pantoea (6), Pseudomonas (16), Streptomyces (1) and Variovorax (2), respectively. Among them, Bacillus spp. and Pseudomonas spp. were more frequently isolated than others.

Co-occurrence network analysis revealed an increased complexity in the NS condition (fig. 1 A to D, and table S5, table S6) when compared with the RNI condition. A higher average degree (avgK) means a more complex network, and a smaller average path distance (GD) means all nodes in the network are closer. The NS fungal and bacterial networks were more complex and their nodes were closer than the RNI fungal and bacterial networks. More positive edges were found in the NS networks comparing with the RNI networks. Overall, the microbial network of NS was composed of more positively interacted microbes to form a more clustered topology structure than the RNI networks.

Most of nodes in the two bacterial networks belonged to 29 bacterial phyla. Among them, Actinobacteria, Bacteroidetes, Firmicutes and Proteobacteria were more dominant than other phyla. Compared with the NS bacterial network, the relative proportions of Actinobacteria, Cyanobacteria, Thaumarchaeota and Verrucomicrobia were all decreased, whereas the relative abundances of Firmicutes, Planctomycetes and Proteobacteria were all increased substantially in the RNI bacterial network. Only $24.4 \%(432 / 1771)$ of nodes were shared by these two bacterial networks, whereas most of nodes (689 for the NS bacterial network and 650 for the RNI bacterial network) were unshared and specific in their own networks (fig. S5A). We further analyzed the 403 nodes belonging to Proteobacteria (fig. S6A). 124 nodes belonging 
to Proteobacteria were enriched in the non-infected soils compared with the nematode infected soils. Among them, Pseudomonas fluorescens can inhibit egg hatching and kill $M$. incognita juveniles [38].

Most of nodes in the two fungal networks belonged to 8 fungal phyla. Among them, Ascomycota, Basidiomycota and Mucoromycota were more dominant than other phyla. Compared with the NS fungal network, the relative proportions of Basidiomycota, Chytridiomycota and Zoopagomycota were all decreased, whereas the relative abundances of Ascomycota and Microsporidia were both increased in the RNI fungal network. 59.5\% of nodes were shared, and 83 nodes ( 38 nodes in the NS network and 45 nodes in the RNI network) were unshared by these two fungal networks (fig. S5B). We further analyzed the nodes belonging to Ascomycota and Basidiomycota. Compared to the NS network, 27 nodes were enriched in the RNI fungal network (fig. S6B). Among them, 8 nodes belonged to Ascomycota, and 9 nodes belonged to Basidiomycota. Rhizophagus irregularis, an arbuscular mycorrhizal fungi, can improve phosphorus uptake and water use efficiency in plants [39]. Phanerochaete chrysosporium is a decomposer in carbon cycle (such as lignin degradation) [40]. These results suggested that the composition of microbial networks had varied considerably in the RNI soils when compared with the NS soils. It seems that the microbial network topology structure and composition were substantially shaped in the root-knot nematode infection soils in this mountain ecosystem.

We further analyzed the keystone microorganisms in bacterial networks (fig. 2A). Seven module hubs (generalists) were found in the NS bacterial network: Acinetobacter gerneri, Granulicella mallensis, Luteimonas mephitis, Niveispirillum irakense, Streptomyces thermoautotrophicus, Terriglobus saanensis and Thiohalobacter thiocyanaticus. Three connectors (generalists) were found in the NS bacterial network: Gloeobacter violaceus, Oerskovia turbata and Rubrobacter radiotolerans. These keystone microorganisms belonged to Proteobacteria (4 module hubs), Acidobacteria (2 module hubs), Actinobacteria (1 module hub, 2 connectors) and Cyanobacteria (1 connector). We have isolated four strains belonged to Acinetobacter and one strain belonged to Streptomyces which all showed high nematicidal activity (mortality $\geq 80 \%$ ) against $M$. incognita (fig. S4A). Twelve module hubs (generalists) were found in the RNI bacterial network: Actibacterium atlanticum, Actinomadura macra, 
Actinopolymorpha alba, Chitinophaga terrae, Enterovibrio calviensis, Gracilibacillus orientalis, Halorubrum distributum, Marvinbryantia formatexigens, Megamonas hypermegale, Rubrobacter xylanophilus, Sphingomonas sanxanigenens and Streptomyces celluloflavus. One connector (generalist) was found in the RNI bacterial network: Streptacidiphilus rugosus. These keystone microorganisms belonged to Proteobacteria (3 module hubs), Actinobacteria (4 module hubs, 1 connector), Bacteroidetes (1 module hub), Firmicutes (3 module hubs), and Euryarchaeota (1 module hub). One module hub (generalist), Ceraceosorus bombacis, was found in the NS fungal network (fig. 2B). Two connectors (generalists) were found in the NS fungal network: Bifiguratus adelaidae and Ogataea parapolymorpha. These three keystone fungi belonged to Basidiomycota (1 module hub), Mucoromycota (1 connector) and Ascomycota (1 connector). Three module hubs (generalists) were found in the RNI fungal network: Amanita muscaria, Paxillus involutus and Trichoderma harzianum. Two connectors (generalists) were found in the RNI fungal network: Hebeloma cylindrosporum and Smittium mucronatum. These keystone fungi belonged to Basidiomycota (2 module hubs, 1 connector), Ascomycota (1 module hub) and Zoopagomycota (1 connector). Keystone microorganisms in the RNI networks were different from those in the NS networks. These keystone microorganisms played important roles in stabilizing microbial network, and different keystone microorganisms indicated different microbial networks in these two different soils.

\section{Functional diversity of the rhizosphere microbiota}

Of the genes retrieved from the metagenome data, 58 to $61 \%$ were assigned to a known function (fig. S1B, C). Among 979,916 genes with associated functions, 3,641 genes and 1611 functions were significantly $(P<0.05)$ enriched in the rhizophere microbiota of the non-infected soils. These genes belonged mainly to the pathways classified as "Energy production and conversion", "Amino acid transport and metabolism", "Signal transduction mechanisms", "Secondary metabolites biosynthesis, transport and catabolism" and "Carbohydrate transport and metabolism".

Co-occurrence function network analysis revealed the average path distance (GD) of the NS function network was smaller than the RNI function network, which means that all the nodes in the NS network are closer than those in the RNI network. Average clustering 
coefficient (avgCC) and modularity of the NS network were higher than the RNI network, indicating the NS function network has more stability and resilience than the RNI network. AvgK of the RNI function network was higher than the NS network (table S7). For the NS functional network, 291 gene nodes $(46.1 \%)$ were overrepresented in the NS condition, 65 gene nodes $(10.3 \%)$ were overrepresented in the RNI condition (fig. 3A). Among those nodes enriched in the NS condition, 9 nodes (COG2175, COG3508, COG4664, COG4663, COG2368, COG2761, COG2854, COG4665, ENOG410XSW0) belonged to Category Q (secondary metabolites biosynthesis, transport, and catabolism). One of them, COG2761 (dithiol-disulfide isomerase), is involved in polyketide biosynthesis. On the other hand, 6 nodes including COG0466 (protease), COG0465 (metalloprotease), COG0330 (protease), COG1219 (protease), COG1220 (protease), and COG0826 (Collagenase) belonged to Category O (posttranslational modification, protein turnover, chaperones). We supposed these enriched genes (nodes) are favorable for increasing nematicidal activity of the NS microbiota. In the RNI functional network, 256 nodes (36.9\%) were overrepresented in the NS condition, 60 nodes $(8.6 \%)$ were overrepresented in the RNI condition (fig. 3B). Among those nodes enriched in the RNI condition, COG3843 (VirD2 components) and COG2948 (protein virB10) are involved in Type IV secretory pathway and belong to Category U (intracellular trafficking, secretion, and vesicular transport). Some gram-negative bacterial pathogens use Type IV secretion system that can translocate DNA or proteins into their eukaryotic host cells to promote infection.

For more detailed resolution of the specific functions associated with cluster of orthologous groups (COG) Category Q and Category G (carbohydrate transport and metabolism), we searched for carbohydrate-active enzymes (CAZymes) and secondary metabolite bio-synthetic gene clusters within the metagenome sequences using dbCAN [41] and antiSMASH [42], respectively. Among Category Q, 41 genes were significantly overrepresented $(P<0.05)$ in the NS microbiota, which are all involved in polyketide biosynthesis (fig. S7A). Polyketides have been shown with antimicrobial and nematicidal activity. Among Category G, 6 genes were significantly overrepresented in the NS microbiota, including chitinase and collagenase (fig. S7B). We also found that proteases and lipases genes are enriched in the NS microbiota (fig. S7C, D). We detected the enzyme activity of the 
nematicidal microbes isolated from the NS rhizosphere soils. 20 nematicidal bacteria and one fungus showed protease activity (fig. S8). 3 nematicidal bacteria showed chitinase activity, and 16 nematicidal bacteria showed lipase activity. Bacillus siamensis J4, Bacillus muralis N17, Pseudomonas mosselii E11 and Microbacterium jejuense G15 had high protease activity. Bacillus amyloliquefaciens W1, Bacillus toyonensis B8, Bacillus cereus B13, Bacillus methylotrophicus M12, Bacillus subtilis J6, Burkholderia cepacia B8 and Pseudomonas simiae E15 showed moderate protease activity. Bacillus amyloliquefaciens W1, Bacillus siamensis J4, Bacillus aryabhattai K2, Bacillus subtilis J6 and Bacillus methylotrophicus M12 showed high lipase activity. Bacillus aryabhattai O13, Bacillus cereus B13, Microbacterium trichothecenolyticum G2, Pantoea dispersa P3 and Variovorax paradoxus G7 showed moderate lipase activity. Bacillus cereus B13, Bacillus licheniformis W15 and Microbacterium trichothecenolyticum G2 showed certain chitinase activity.

Our antiSMASH analysis for secondary metabolites revealed a total of 6866 biosynthetic gene clusters (BGCs) in the NS microbiota. Among them, BGCs associated with the biosynthesis of terpene, nonribosomal peptides (NRPS), arylpolyene, bacteriocin, betalactone and polyketides were more dominant than other BGCs. Genes abundances of terpene, NRPS, arylpolyene, bacteriocin, betalactone, polyketides, acyl amino acids, hglEKS, hserlactone, resorcinol, siderophore, phosphonate, ectoine, ladderane, thiopeptide, indole, TfuA, butyrolactone and LAP in the NS microbiota were higher than the RNI microbiota (fig. S9).

Co-occurrence network analysis of BGCs genes revealed rhizomide, anabaenopeptin, icosalide, TP1161, APE Vf, lysobactin, nosiheptide, malleobactin, flexirubin and bicornutin had high abundances in two groups (fig. 3C, D). Altogether, 49 BGCs were overrepresented in the NS microbiota. APE Vf, anabaenopeptin, bicornutin, enduracidin, glidobactin, icosalide, lysobactin, myxochromide and rhizomide were enriched in the NS microbiota as compared to the RNI microbiota (fig. S10). APE Vf and bicornutin were significantly overrepresented $(P<$ 0.05 ) in the NS microbiota comparing with the RNI microbiota.

\section{Untargeted Metabolomics analysis of the rhizosphere microbiota}

PCA analysis showed that metabolites composition of the NS rhizosphere microbiota were different from the RNI rhizosphere microbiota (fig. S11A, B). Partial least squares 
discrimination analysis (PLS-DA) showed the RNI samples were well separated from the NS samples (fig. 4A, B). Different metabolites analysis showed 102 metabolites were significantly different between the RNI and NS rhizosphere microbiota (fig. 4 C, D; fig. S12A, B). 67 metabolites were overrepresented in the RNI samples comparing with the NS samples, such as alternariol (Com_388_pos). 35 metabolites were overrepresented in the NS samples comparing the RNI samples, such as acetophenone (Com_401_pos) $(\log 2$ FoldChange $=1.083$, $P<0.05)$ and indole-3-acetic acid $($ Com_107_neg) $(\log 2$ FoldChange $=1.583, P<0.05)$ (fig. 4 E, F).

We further analyzed the correlation between the number of $M$. incognita and the metabolites of rhizosphere microbiota. Total 21 metabolites were significantly $(P<0.05)$ negatively correlated with the number of $M$. incognita (table S8), such as acetophenone. We detected nematicidal activity of these metabolites, and found that acetophenone showed high nematicidal activity. $13.79 \%, 14.96 \%, 21.32 \%, 26.41 \%, 54.39 \%, 81.42 \%, 90.71 \% \quad$ and $100 \%$ mortality were respectively observed when acetophenone was used at a concentration of $0.41,0.46,0.52,0.59,0.69,0.82,1.03$ and $2.06 \mu \mathrm{g} / \mathrm{ml}$ for treatment of $M$. incognita $\mathrm{J} 2$ juveniles for $24 \mathrm{~h}$ (fig. S13A). $\mathrm{LC}_{50}$ and $\mathrm{LC}_{90}$ value of acetophenone was $0.66 \mu \mathrm{g} / \mathrm{ml}$ and 1.01 $\mu \mathrm{g} / \mathrm{ml}$, respectively. Indole-3-acetic acid showed a low nematicidal activity, $2.95 \%$ mortality of $M$. incognita was observed when indole-3-acetic acid was used at $1 \mathrm{mg} / \mathrm{ml}$ for treatment of nematode for $24 \mathrm{~h}$. Other metabolites showed no nematicidal activity against $M$. incognita (data not shown). Besides, acetophenone can also repel M. incognita. The avoidance index of acetophenone was 0.11 and 0.18 for the dose of 2 and $4 \mu 1$, respectively (fig. S13B).

We constructed the network including acetophenone, the microbes enriched in the NS microbiota and the keystone microorganisms in the NS network (fig. 5). Bacillus sp. was positively interacted with acetophenone. Rhizobium was negatively interacted with acetophenone. Pantoea and Pseudomonas were positively interacted with Bacillus. We isolated several Bacillus stains from the NS rhizosphere soils and found that these Bacillus spp. isolates showed different nematicidal activity $(2.5-98.8 \%$ mortality) towards $M$. incognita (fig. S13C). B. amyloliquefaciens W1 could kill 98.8\% of M. incognita. We further detected the metabolites in $\mathrm{W} 1$ culture, and found $\mathrm{W} 1$ could produce acetophenone $(\mathrm{C} 8 \mathrm{H} 8 \mathrm{O})$ (table S9). Several other metabolites involved in the phenylalanine metabolism pathway were 
also detected in the $\mathrm{W} 1$ culture, such as 2-phenylacetamide, 2-hydroxyphenylacetic acid, phenylpyruvic acid and phenylacetaldehyde (table S9, fig. S13D).

\section{Discussion}

Soil properties of the NS soils were very different from the RNI soils. Invertase involved in soil carbon cycle is a characterization of soil fertility level. Catalase is associated with soil respiration and microbial activity. Here, these two enzymes activities in the NS rhizosphere soils were higher than those in the RNI soils, suggesting that microbial activity in the non-infected soils is higher than that in the root-knot nematode infected soils. Soil acidification was favorable for the hatching of root-knot nematode eggs and could increase the density of second instar larvae in soil, so aggravated the occurrence of root-knot nematode disease [43]. The $\mathrm{pH}$ value of the RNI soils was much lower than the NS soils, thereby soil acidification in this mountain region possibly aggravated the root-knot nematode disease. Soil total porosity and mean weight diameter of the NS soils were higher than the RNI soils, implying the non-infected soils were more loose to be favorable for improving microbial activity. Instead, the root-knot nematode infected soils showed compaction phenomenon (e.g. increasing soil bulk density, decreasing porosity and water content), thus lowered crop performance via stunting the aboveground growth coupled with reduced root growth [44]. Alterations in soil porosity after compaction strongly limited the air and water conductivity, so soil compaction could reduce microbial abundance and persistently alter the structure of microbiota [45]. Soil calcium plays roles in plant tolerance to the stresses like acidosis, toxic metals, osmosis and pathogen infection [46, 47]. Thereby, lower calcium content in the RNI soils may aggravate nematodes infection in the tobacco roots. Iron $(\mathrm{Fe})$ is an essential element, which acts as an important co-factor for the enzymes involved in regulating various metabolic processes in plants (e.g. chlorophyll synthesis), for plant growth and development [48]. Here, low iron content in the RNI soils may have a negative impact on plant growth and thus make tobacco more susceptible to pathogens infection (e.g. M. incognita). Indeed, catalase activity, $\mathrm{pH}, \mathrm{STP}, \mathrm{MWD}, \mathrm{Ca}$ and $\mathrm{Fe}$ were all negatively correlated to the number of $M$. incognita significantly. Changes of these soil properties possibly are related to the aggravated root-knot nematode disease in the RNI soils. 
Microorganisms can attack and kill root-knot nematodes by diverse processes such as capturing, parasitizing, and producing toxins and enzymes. Previous reports have shown that nematicidal antagonists such as Comamonas, Paenibacillus, Pseudomonas, Variovorax, Virgibacillus and Achromobacter xylosoxidans, could produce nematicidal volatile organic compounds against Meloidogyne spp. [5, 49-51]. Aspergillus, Lactobacillus brevis and Lysobacter capsici showed strong nematicidal activity and could inhibit the egg hatch of Meloidogyne spp., and their nematicidal activities were derived mainly from organic acids [7, 52, 53]. Many soil microorganisms such as Acinetobacter spp., Microbacterium sp., Enterobacter spp., Pantoea agglomerans, Pseudomonas putida and Sinorhizobium fredii, could induce systemic resistance in plants against the root-knot nematodes infection [54-57]. Alcaligenes faecalis showed toxicity against $M$. incognita by producing an extracellular serine protease, which can damage the intestines of nematode [37]. Enterobacter asburiae displayed promising nematicidal activity against $M$. incognita, and its nematicidal virulence factor was also determined as the proteolytic enzymes [58]. Rhizobacteria such as Burkholderia gladioli, Delftia acidovorans, Rhizobium etli, Pseudomonas spp. and Bacillus spp. could significantly reduce the hatching of eggs, the numbers of second stage juvenile of Meloidogyne and root galls [6, 59-61]. Escherichia coli secreted small molecules including indole, indole-3-carboxaldehyde and indole-3-acetic acid, as virulence factors to kill nematode [62]. Pseudoxanthomonas japonensis produced 3 - methoxycyclobutane - 1, 2 - dione to kill $M$. incognita [63]. Many nematocidal antibiotics and metabolites (e.g. jietacin, polyketides, lactones) were produced by Streptomyces spp. to reduce root galls and inhibit egg hatching [64-66]. Metagenomics analysis showed these nematicidal microorganisms were enriched in the NS soils comparing with the RNI soils, and from the NS soils we got 62 bacterial isolates and two fungal isolates belonging to these genera with high nematicial activity. Bacillus spp. and Pseudomonas spp. were more frequently isolated, indicating they may play key roles in protecting plants against nematode infection. The bacterial community richness and diversity in root-knot nematode diseased soils was significantly different from that in healthy soils [67]. This was also observed in our findings that a significant difference in the microbial community composition was found between the NS and RNI rhizospheric soils, suggesting the rhizospheric microbial community structure was shifted in the RNI soils compared to the 
NS soils.

Higher average connectivity (i.e. average links per node in the network) means a more complex network. A smaller average geodesic distance means all the nodes in the network are closer $[12,25]$. We found the NS fungal and bacterial networks were more complex and their nodes were closer than the RNI networks. The increased complexity of the NS networks was reflected by the increased average connectivity, as well as the shorter geodesic distances, indicating the NS rhizosphere microbiota has a greater potential for interactions and niche-sharing each other. Rhizosphere microorganisms are very important for protecting plants against pathogens infection. Highly connected networks, like those in the NS samples, are more effective for the rhizosphere microbiota to defense pathogens invasion [11, 27]. On the contrary, the interactions or niche sharing are minimal in the RNI networks relative to the NS networks, indicating the ecological networks have shifted in the RNI soils compared to the NS soils. Changes in environmental properties (e.g. $\mathrm{pH}$, hydrological characteristics, etc) can alter ecological networks $[68,69]$. Resource and food availability are important drivers of network structures [70], so the increased complexity of microbial networks was likely due to the increased amount of carbon input in soil [12]. Large number of nematode in the RNI soils (1091 nematodes per gram of soil) consumed large amounts of food and energy which may lead to consequent shortage of resource and food for microbial growth, thus decrease the complexity of microbial network. Interestingly, more positive edges were found in the NS networks than the RNI networks, indicating the potential for extensive mutualistic interactions among microorganisms assembles in the NS rhizosphere [71]. Negative co-occurrence patterns (co-exclusion) predominated in the RNI networks, suggesting that root-knot nematode infection triggered microbial competition and antagonistic interactions in the RNI soils [72].

Ten keystone microorganisms were found in the NS bacterial network. These keystone species are critical in maintaining the NS network structure and function, and ecosystem stability [27, 73]. Terriglobus saanensis and Granulicella mallensis both belong to Acidobacteria and can hydrolyze diverse carbohydrates including complex polysaccharides [74]. Xylanosome produced by Oerskovia turbata exhibits powerful degradative ability against xylan and hemicellulose [75]. Streptomyces thermoautotrophicus is able to fix $\mathrm{N}_{2}$ [76]. 
These keystone microorganisms participate in carbon and nitrogen cycles, possibly supply carbon and nitrogen resources for other microorganisms in the NS network. Occurrence of these keystone species may be indicative of the influence of soil nutrients and root exudates on microbial co-occurrence relationships in soil. The actinomycetes such as Rubrobacter radiotolerans produces two dimeric indole derivatives with acetylcholinesterase inhibitory activity [77]. The acetylcholinesterase inhibitors, e.g. oxamyl and avermectin, cause initial hyperactivity of nematode juveniles followed by a progressive decline in movement and then a final loss of activity [78]. Thereby, as keystone specie, $R$. radiotolerans may play important roles in control of root-knot nematode disease. The keystone taxa shifted as conditions (land uses, pathogen infection) changed [27, 79]. Here, the keystone species of the RNI networks were different from those of the NS networks, indicating the keystone species of microbial network were altered in the RNI soil.

Microorganisms can kill nematodes by producing extracellular enzymes, including serine protease, metalloproteinase, collagenase, lipase and chitinase, which can degrade the nematode cuticle and eggshell $[80,81]$. We found the genes of chitinase, collagenase, lipase and protease were significantly overrepresented in the NS microbiota comparing with the RNI microbiota. 291 genes were overrepresented in the NS functional network. Among these genes, 4 nodes (COG0466, COG0330, COG1219, COG1220) encode protease, COG0465 encodes metalloprotease, and COG0826 encodes collagenase. We isolated 20 nematicidal bacterial strains and one fungal strain with protease activity, 3 bacterial strains showed chitinase activity, and 16 bacterial strains showed lipase activity. These extracellular enzymes might act as important nematicidal virulence factors in the NS rhizosphere microbiota.

Polyketides, an important class of biologically active compounds, possess potent nematicidal activity [66]. Among nodes enriched in the NS function network, COG2761 (dithiol-disulfide isomerase) is involved in polyketide biosynthesis. Total 41 genes of Category Q were overrepresented in the NS microbiota, which are involved in polyketide biosynthesis. The toxic secondary metabolites such as anabaenopeptins could reduce worm reproduction and shorten worm lifespan of the model nematode Caenorhabditis elegans [82]. The secondary metabolites (e.g. bicornutin, enduracidin, glidobactins, icosalide, lysobactin, rhizomide) display antimicrobial activity. Our antiSMASH analysis revealed a total of 6866 
BGCs in the NS rhizosphere microbiota. Co-occurrence function network analysis revealed that 49 BGCs were all overrepresented in the NS rhizosphere microbiota including APE Vf, anabaenopeptin, bicornutin, enduracidin, glidobactin, icosalide, lysobactin, myxochromide and rhizomide, indicating these antibiotics are possible as nematicidal antibiotics to protect plants from nematode infection.

We found the metabolites of the NS microbiota were different from those of the RNI microbiota based on the untargeted metabolomics analysis. Alternariol, a kind of mycotoxin produced by the phytopathogenic fungi Alternaria, acts as a virulence and colonization factor during their infection of plants [83]. Interestingly, alternariol was enriched in the RNI microbiota, indicating the tobacco plants were also infected by Alternaria in the RNI soils that was consistent with our investigations (data unshown). The co-infection of Alternaria and root-knot nematode may aggravate the death of plants.

Acetophenone was overrepresented in the NS samples. Two derivatives of acetophenone, 4-nitroacetophenone and 4-iodoacetophenone, were previously reported with nematicidal activity against $M$. incognita, with $\mathrm{EC}_{50}$ values of 12 and $15 \mathrm{mg} / \mathrm{L}$ respectively [84]. In this study, we found that acetophenone was also very effective for killing nematodes. Not only that, its nematicidal activity $\left(\mathrm{LC}_{50}=0.66 \mathrm{mg} / \mathrm{L}\right)$ was even much higher than its derivatives of 4-nitroacetophenone and 4-iodoacetophenone. Indeed, acetophenone showed a strong nematicidal activity. After treatment for $24 \mathrm{~h}, 0.66 \mu \mathrm{g} / \mathrm{ml}$ and $1.01 \mu \mathrm{g} / \mathrm{ml}$ of acetophenone could kill $50 \%$ and $90 \%$ of $M$. incognita $\mathrm{J} 2$ juveniles, respectively, but how acetophenone kill nematode is needed to be further investigated. The nematicidal activity of acetophenone was even higher than the popularly used commercial nematicide avermectin $\left(\mathrm{LC}_{50}=2.0 \mathrm{mg} / \mathrm{L}\right)$ [85]. Moreover, as nematodes resistance to avermectins is on the rise, alternatives or new products with enhanced potency and high activity are urgently needed [86, 87]. Considering that acetophenone has the advantages of simple structure, high efficiency and safety [88], it is a very potential nematicide candidate in the future. Besides for directly killing nematode, we found that acetophenone could strongly repel $M$. incognita as a repellent of root-knot nematode. Interestingly, acetophenone was also reported with strong repellency activity to some pests such as the kissing bug Rhodnius prolixus at a low dose (1\%) [89], and as an anti-attractant for the pest western pine beetle, Dendroctonus brevicomis LeConte [90]. 
Addition to acetophenone, indole-3-acetic acid (IAA) was also overrepresented in the NS samples. It is known that IAA produced by microbes can promote plant growth. Thereby, overrepresent of IAA in the NS rhizosphere soils was favorable for improving tobacco growth.

Co-occurrence network analysis found Bacillus was positively interacted with acetophenone. We isolated nematicidal Bacillus spp. from the NS rhizosphere soils. Among these isolates, B. amyloliquefaciens $\mathrm{W} 1$ culture could kill $98.8 \%$ of $M$. incognita J2 juveniles and could produce acetophenone. This study firstly found the nematicidal activity of acetophenone produced by B. amyloliquefaciens. This new discovery has broken new ground in exploration of the nematicidal metabolites from microbes through combining multi-omics analysis as well as culture-dependent technology. Interestingly, several intermediary metabolites in phenylalanine metabolism pathway were also found in the W1 culture, including 2-phenylacetamide, 2-hydroxyphenylacetic acid, phenylpyruvic acid and phenylacetaldehyde. We supposed that acetophenone may be one of the metabolites in phenylalanine metabolism, and the pathway of acetophenone production was needed to be investigated asap. Pantoea and Pseudomonas could induce systemic resistance against root-knot nematodes [54]. Here, we found that Pantoea and Pseudomonas positively interacted with Bacillus, indicating these nematicidal bacteria could cooperate together to kill nematode by multiple nematicidal mechanisms. Previous study has demonstrated that a native consortium of five native bacterial isolates protected their host plant from a fungal sudden-wilt disease [91]. Here, a consortium of Bacillus, Pantoea and Pseudomonas isolates may effectively control M. incognita via complementary traits.

\section{Conclusion}

We compared the non-infected and root-knot nematode infected tobacco rhizosphere microbiota by metagenomics and metabolomics analysis. Rhizosphere microbiota was altered accompanying with root-knot nematode infection. The RNI microbial community, microbial network, functional network and metabolites were quite different from the NS microbiota. The NS microbial networks were more complex than the RNI networks. Nematicidal microbes were enriched in the NS rhizosphere microbiota. Potential nematicidal metabolites, antibiotics and extracellular enzymes, which are the nematicidal virulence factors, were 
overrepresented in the NS microbiota. Consistently, nematicidal bacterial and fungal isolates were got from the NS rhizosphere soils, which could produce extracellular enzymes. Importantly, the nematicidal B. amyloliquefaciens $\mathrm{W} 1$ could produce acetophenone to kill and repel root-knot nematode. Collectively, combination of multi-omics analysis and culture-dependent technology is powerful for finding nematicidal microorganisms and metabolites from soil.

\section{Declarations}

\section{Ethics approval and consent to participate}

Not applicable.

\section{Consent for publication}

Not applicable.

\section{Availability of data and material}

All sequencing data has been submitted to the NCBI repository and can be accessed via the following accession numbers: whole-genome shotgun sequencing PRJNA657468, PRJNA657384, PRJNA657444.

\section{Competing interests}

The authors declare that they have no competing interests.

\section{Funding}

This work was supported by the National Natural Science Foundation of China (31870030).

\section{Authors' contributions}

Conceptualization: X.Z., and G.Q. Investigation: C.L., W.Y., and S.R. Data curation: X.Z., and C.L. Writing-review and editing: X.Z., and G.Q. Funding acquisition: G.Q. The author(s) read and approved the final manuscript.

\section{Acknowledgements}

Thank you to Dr. Wang R. and Tan J for the assistance with soil samples collecting. 


\section{References}

1. Davies LJ, Elling AA. Resistance genes against plant-parasitic nematodes: a durable control strategy? Nematology. 2015, 17: 249-263.

2. Huang Y, Ma L, Fang DH, Xi JQ, Zhu ML, Mo MH, Zhang KQ, Ji YP. Isolation and characterisation of rhizosphere bacteria active against Meloidogyne incognita, Phytophthora nicotianae and the root knot-black shank complex in tobacco. Pest Manag Sci. $2015,71:$ 415-22.

3. Du B, Xu Y, Dong H, Li Y, Wang J. Phanerochaete chrysosporium strain B-22, a nematophagous fungus parasitizing Meloidogyne incognita. PLoS One. 2020, 15: $\mathrm{e} 0216688$.

4. Castillo JD, Vivanco JM, Manter DK. Bacterial microbiome and nematode occurrence in different potato agricultural soils. Microb Ecol. 2017, 74: 888-900.

5. Cheng W, Yang J, Nie Q, Huang D, Yu C, Zheng L, Cai M, Thomashow LS, Weller DM, Yu Z, Zhang J. Volatile organic compounds from Paenibacillus polymyxa KM2501-1 control Meloidogyne incognita by multiple strategies. Sci Rep. 2017, 7: 16213.

6. Turatto MF, Dourado FDS, Zilli JE, Botelho GR. Control potential of Meloidogyne javanica and Ditylenchus spp. using fluorescent Pseudomonas and Bacillus spp. Braz J Microbiol. 2018, 49: 54-58.

7. Seo HJ, Park AR, Kim S, Yeon J, Yu NH, Ha S, Chang JY, Park HW, Kim JC. Biological control of root-knot nematodes by organic acid-producing Lactobacillus brevis WiKim0069 isolated from Kimchi. Plant Pathol J. 2019, 35: 662-673.

8. Goswami BK, Pandey RK, Singh S, Rathour KS. Biopesticides: an ecolofriendly, sustainable and cost effective approach for integrated disease and insect pest management of agricultural crops. In: Ahamad S., editor. Plant Disease Management for Sustainable Agriculture. Daya Publishing House; Delhi: 2009. pp. 292-298.

9. Deketelaere S, Tyvaert L, França SC, Höfte M. Desirable traits of a good biocontrol agent against Verticillium wilt. Front Microbiol. 2017, 8: 1186.

10. Chen S, Qi G, Luo T, Zhang H, Jiang Q, Wang R, Zhao X. Continuous-cropping tobacco caused variance of chemical properties and structure of bacterial network in soils. Land Degrad Dev. 2018, 29: 4106-20. 
11. Qi G, Ma G, Chen S, Lin C, Zhao X. Microbial network and soil properties are changed in bacterial wilt-susceptible soil. Appl Environ Microbiol. 2019, 85: e00162-19.

12. Zhou J, Deng Y, Luo F, He Z, Yang Y. Phylogenetic molecular ecological network of soil microbial communities in response to elevated $\mathrm{CO}_{2}$. mBio. 2011, 2: e00122-11.

13. Faust K, Raes J. Microbial interactions: from networks to models. Nat Rev Microbiol. 2012, 10: 538-550.

14. Zhou X, Wang JT, Wang WH, Tsui CK, Cai L. Changes in bacterial and fungal microbiomes associated with tomatoes of healthy and infected by Fusarium oxysporum $\mathrm{f}$. sp. Lycopersici. Microb Ecol. 2020.

15. Grim CM, Luu GT, Sanchez LM. Staring into the void: demystifying microbial metabolomics. FEMS Microbiol Lett. 2019, 366: fnz135.

16. Zhao YL, Ruan WB, Yu L, Zhang JY, Fu JM, Shain EB, Huang XT, Wang JG. Combining maxRatio analysis with real-time PCR and its potential application for the prediction of Meloidogyne incognita in field samples. J Nematol. 2010, 42: 166-72.

17. Bao SD. Soil and agricultural chemistry analysis (eds Jiang, R. F. et al.) (China Agriculture Press, 2013).

18. Wang R, Zhang H, Sun L, Qi G, Chen S, Zhao X. Microbial community composition is related to soil biological and chemical properties and bacterial wilt outbreak. Sci Rep. 2017, 7: 343 .

19. Bielders CL, De Backer LW, Delvaux B. Particle density of volcanic soils as measured with a gas pycnometer. Soil Sci Soc Am J. 1990, 54: 822-826.

20. Qin N, Yang F, Li A, Prifti E, Chen Y, Shao L, et al. Alterations of the human gut microbiome in liver cirrhosis. Nature. 2014, 513: 59-64.

21. Li W, Godzik A. Cd-hit: a fast program for clustering and comparing large sets of protein or nucleotide sequences. Bioinformatics. 2006, 22: 1658-9.

22. Buchfink B, Xie C, Huson DH. Fast and sensitive protein alignment using DIAMOND. Nat Methods. 2015, 12: 59-60.

23. White JR, Nagarajan N, Pop M. Statistical methods for detecting differentially abundant features in clinical metagenomic samples. PLoS Comput Biol. 2009, 5: e1000352.

24. Luo F, Zhong JX, Yang YF, Scheuermann RH, Zhou JZ. Application of random matrix 
theory to biological networks. Phys Lett A. 2006, 357: 420-423.

25. Deng Y, Jiang YH, Yang YF, He ZL, Luo F, Zhou JZ. Molecular ecological network analyses. BMC Bioinformatics. 2012, 13: 113.

26. Bascompte J, Stouffer DB. The assembly and disassembly of ecological networks. Philos Trans R Soc Lond B Biol Sci. 2009, 364: 1781-1787.

27. Lu L, Yin S, Liu X, Zhang $\mathrm{W}, \mathrm{Gu} \mathrm{T}$, Shen Q, Qiu H. Fungal networks in yield-invigorating and -debilitating soils induced by prolonged potato monoculture. Soil Biol Biochem. 2013, 65: 186-194.

28. Guimera R, Nunes Amaral LA. Functional cartography of complex metabolic networks. Nature. 2005, 433: 895-900.

29. Want EJ, Wilson ID, Gika H, Theodoridis G, Plumb RS, Shockcor J, Holmes E, Nicholson JK. Global metabolic profiling procedures for urine using UPLC-MS. Nature protocols. 2010, 5: 1005-1018.

30. Kieffer DA, Piccolo BD, Vaziri ND, Liu S, Lau WL, Khazaeli M, Nazertehrani S, Moore ME, Marco ML, Martin RJ, Adams SH. Resistant starch alters gut microbiome and metabolomics profiles concurrent with amelioration of chronic kidney disease in rats. Am J Physiol Renal Physiol. 2016, 310: F857-71.

31. Gao H, Qi G, Yin R, Zhang H, Li C, Zhao X. Bacillus cereus strain S2 shows high nematicidal activity against Meloidogyne incognita by producing sphingosine. Sci. Rep. 2016, 6: 28756.

32. Luo T, Hou S, Yang L, Qi G, Zhao X. Nematodes avoid and are killed by Bacillus mycoides-produced styrene. J Invertebr Pathol. 2018, 159: 129-136.

33. Borneman J, Hartin RJ. PCR primers that amplify fungal rRNA genes from environmental samples. Appl Envir Microbiol. 2000, 66: 4356-4360.

34. Larousse M, Rancurel C, Syska C, Palero F, Etienne C, Industri B, Nesme X, Bardin M, Galiana E. Tomato root microbiota and Phytophthora parasitica-associated disease. Microbiome. 2017, 5: 56.

35. Letunic I, Bork P. Interactive Tree Of Life v2: Online annotation and display of phylogenetic trees made easy. Nucleic Acids Res. 2011, 39: W475-W478.

36. Ronquist F, Teslenko M, van der Mark P, Ayres DL, Darling A, Höhna S, Larget B, Liu L, 
Suchard MA, Huelsenbeck JP. MrBayes 3.2: efficient Bayesian phylogenetic inference and model choice across a large model space. Syst Biol. 2012, 61: 539-42.

37. Ju S, Lin J, Zheng J, Wang S, Zhou H, Sun M. Alcaligenes faecalis ZD02, a novel nematicidal bacterium with an extracellular serine protease virulence factor. Appl Environ Microbiol. 2016, 82: 2112-2120.

38. Siddiqui IA, Haas D, Heeb S. Extracellular protease of Pseudomonas fluorescens CHA0, a biocontrol factor with activity against the root-knot nematode Meloidogyne incognita. Appl Environ Microbiol. 2005, 71: 5646-9.

39. Le Pioufle O, Ganoudi M, Calonne-Salmon M, Ben Dhaou F, Declerck S. Rhizophagus irregularis MUCL 41833 improves phosphorus uptake and water use efficiency in maize plants during recovery from drought stress. Front Plant Sci. 2019, 10: 897.

40. Hong CY, Ryu SH, Jeong H, Lee SS, Kim M, Choi IG. Phanerochaete chrysosporium multienzyme catabolic system for in vivo modification of synthetic lignin to succinic acid. ACS Chem Biol. 2017, 12: 1749-1759.

41. Yin Y, Mao X, Yang J, Chen X, Mao F, Xu Y. dbCAN: a web resource for automated carbohydrate-active enzyme annotation. Nucleic Acids Res. 2012, 40: W445-51.

42. Weber T, Blin K, Duddela S, Krug D, Kim HU, Bruccoleri RE, et al. antiSMASH 3.0-A comprehensive resource for the genome mining of biosynthetic gene clusters. Nucleic Acids Res, 2015, 43: W237-W243.

43. Gu DY. Effects of soil acidification and $\mathrm{CaCN} \_2$ treatment on growth and development of cucumber and root-knot nematode disease. Shandong Agricultural University, Master's thesis, 2006.

44. Shah AN, Tanveer M, Shahzad B, Yang G, Fahad S, Ali S, Bukhari MA, Tung SA, Hafeez A, Souliyanonh B. Soil compaction effects on soil health and cropproductivity: an overview. Environ Sci Pollut Res Int. 2017, 24: 10056-10067.

45. Hartmann M, Niklaus PA, Zimmermann S, Schmutz S, Kremer J, Abarenkov K, Lüscher P, Widmer F, Frey B. Resistance and resilience of the forest soil microbiome to logging-associated compaction. ISME J. 2014, 8: 226-44.

46. Jiang T, Zhan X, Xu Y, Zhou L, Zong L. Roles of calcium in stress-tolerance of plants and its ecological significance.Ying Yong Sheng Tai Xue Bao. 2005, 16: 971-6. 
47. Springer YP, Hardcastle BA, Gilbert GS. Soilcalcium and plant disease in serpentine ecosystems: a test of the pathogen refuge hypothesis. Oecologia. 2007, 151: 10-21.

48. Mahender A, Swamy BPM, Anandan A, Ali J. Tolerance of iron-deficient and -toxic soil conditions in rice. Plants. 2019, 8: 31 .

49. Xu YY, Lu H, Wang X, Zhang KQ, Li GH. Effect of volatile organic compounds from bacteria on nematodes. Chem Biodivers. 2015, 12: 1415-21.

50. Wolfgang A, Taffner J, Guimarães RA, Coyne D, Berg G. Novel strategies for soil-borne diseases: exploiting the microbiome and volatile-based mechanisms toward controlling Meloidogyne-based disease complexes. Front Microbiol. 2019, 10: 1296.

51. Huang D, Yu C, Shao Z, Cai M, Li G, Zheng L, Yu Z, Zhang J. Identification and characterization of nnematicidal volatile organic compounds from deep-sea Virgibacillus dokdonensis MCCC 1A00493. Molecules. 2020, 25. pii: E744.

52. Lee YS, Naning KW, Nguyen XH, Kim SB, Moon JH, Kim KY. Ovicidal activity of lactic acid produced by Lysobacter capsici YS1215 on eggs of root-knot nematode, Meloidogyne incognita. J Microbiol Biotechnol. 2014, 24: 1510-5.

53. Lee S, Lee K, Chun H, Ha S, Gwak H, Kim H, Lee J, Choi H, Kim H, Shin T, Park H, Kim J. Process development of oxalic acid production in submerged culture of Aspergillus niger F22 and its biocontrol efficacy against the root-knot nematode Meloidogyne incognita. Bioprocess Biosyst Eng. 2018, 41: 345-352.

54. Munif A, Hallmann J, Sikora RA. Induced systemic resistance of selected endophytic bacteria against Meloidogyne incognita on tomato. Meded Rijksuniv Gent Fak Landbouwkd Toegep Biol Wet. 2001, 66: 663-9.

55. Tian F, Wang Y, Zhu X, Chen L, Duan Y. Effect of Sinorhizobium fredii strain Sneb183 on the biological control of soybean cyst nematode in soybean. J Basic Microbiol. 2014, 54: $1258-63$.

56. Topalović O, Elhady A, Hallmann J, Richert-Pöggeler KR, Heuer H. Bacteria isolated from the cuticle of plant-parasitic nematodes attached to and antagonized the root-knot nematode Meloidogyne hapla. Sci Rep. 2019, 9:11477.

57. Topalović O, Bredenbruch S, Schleker ASS, Heuer H. Microbes attaching to endoparasitic phytonematodes in soil trigger plant defense upon root penetration by the 
nematode. Front Plant Sci. 2020, 11: 138.

58. Oh M, Han JW, Lee C, Choi GJ, Kim H. Nematicidal and plant growth-promoting activity of Enterobacter asburiae HK169: genome analysis provides insight into its biological activities. J Microbiol Biotechnol. 2018, 28: 968-975.

59. Mahdy M, Hallmann J, Sikora RA. Influence of plant species on the biological control activity of the antagonistic rhizobacterium Rhizobium etli strain G12 toward the root-knot nematode, Meloidogyne incognita. Meded Rijksuniv Gent Fak Landbouwkd Toegep Biol Wet. 2001, 66: 655-62.

60. Han JW, Oh M, Choi GJ, Kim H. Genome sequence of Delftia acidovorans HK171, a nematicidal bacterium isolated from tomato roots. Genome Announc. 2017, 5(9). pii: e01746-16.

61. Khanna K, Sharma A, Ohri P, Bhardwaj R, Abd Allah EF, Hashem A, Ahmad P. Impact of plant growth promoting rhizobacteria in the orchestration of Lycopersicon esculentum mill. Resistance to plant parasitic nematodes: a metabolomic approach to evaluate defense responses under field conditions. Biomolecules. 2019, 9(11). pii: E676.

62. Bommarius B, Anyanful A, Izrayelit Y, Bhatt S, Cartwright E, Wang W, Swimm AI, Benian GM, Schroeder FC, Kalman D. A family of indoles regulate virulence and Shiga toxin production in pathogenic E. coli. PLoS One. 2013, 8: e54456.

63. Hu Y, Li J, Li J, Zhang F, Wang J, Mo M, Liu Y. Biocontrol efficacy of Pseudoxanthomonas japonensis against Meloidogyne incognita and its nematostatic metabolites. FEMS Microbiol Lett. 2019, 366(2).

64. Tsuzuki K, Yan FS, Otoguro K, Omura S. Synthesis and nematocidal activities of jietacin A and its analogs. J Antibiot (Tokyo). 1991, 44: 774-84.

65. Li JS, Zhang H, Zhang SY, Wang HY, Zhang J, Chen AL, Wang JD, Xiang WS. New macrocyclic lactones with acaricidal and nematocidal activities from a genetically engineered strain Streptomyces bingchenggensis BCJ60. J Asian Nat Prod Res. 2017, 19: 339-346.

66. Feng Y, Yu Z, Zhang S, Xue Z, Huang J, Zhang H, Wan X, Chen A, Wang J. Isolation and characterization of new 16-membered macrolides from the aveA3 gene replacement mutant 
strain Streptomyces avermitilis TM24 with acaricidal and nematicidal activities. J Agric Food Chem. 2019, 67: 4782-4792.

67. Huang K, Jiang Q, Liu L, Zhang S, Liu C, Chen H, Ding W, Zhang Y. Exploring the key microbial changes in the rhizosphere that affect the occurrence of tobacco root-knot nematodes. AMB Express. 2020, 10: 72.

68. Tylianakis JM, Tscharntke T, Lewis OT. Habitat modification alters the structure of tropical host-parasitoid food webs. Nature. 2007, 445: 202-205.

69. Barberan A, Bates ST, Casamayor EO, Fierer N. Using network analysis to explore co-occurrence patterns in soil microbial communities. ISME J. 2012, 6: 343-351.

70. Foster EA, Franks DW, Morrell LJ, Balcomb KC, Parsons KM, van Ginneken A. et al. Social network correlates of food availability in an endangered population of killer whales. Orcinus orca. Anim. Behav. 2012, 83: 731-736.

71. Ren D, Madsen JS, Sorensen SJ, Burmolle M. High prevalence of biofilm synergy among bacterial soil isolates in cocultures indicates bacterial interspecific cooperation. ISME J. 2015, 9: 81-89.

72. Berry D, Widder S. Deciphering microbial interactions and detecting keystone species with co-occurrence networks. Front Microbiol. 2014, 5: 1-14.

73. Olesen J, Bascompte J, Dupont Y, Jordano P. The modularity of pollination networks. Proc. Natl Acad. Sci. USA. 2007, 104: 19891-19896.

74. Rawat SR, Männistö MK, Bromberg Y, Häggblom MM. Comparative genomic and physiological analysis provides insights into the role of Acidobacteria in organic carbon utilization in Arctic tundra soils. FEMS Microbiol Ecol. 2012, 82: 341-55.

75. Dou TY, Liu WJ, Chen J. Isolation, substrate specificity, and subunit characterization of the xylanosomes produced by Oerskovia turbata JCM 3160. Curr Microbiol. 2020, 77 : 924-930.

76. Ribbe M, Gadkari D, Meyer O. $\mathrm{N}_{2}$ fixation by Streptomyces thermoautotrophicus involves a molybdenum-dinitrogenase and a manganese-superoxide oxidoreductase that couple $\mathrm{N}_{2}$ reduction to the oxidation of superoxide produced from $\mathrm{O}_{2}$ by a molybdenum-CO dehydrogenase. J Biol Chem. 1997, 272: 26627-33.

77. Li JL, Huang L, Liu J, Song Y, Gao J, Jung JH, Liu Y, Chen G. Acetylcholinesterase 
inhibitory dimeric indole derivatives from the marine actinomycetes Rubrobacter radiotolerans. Fitoterapia. 2015, 102: 203-7.

78. Wright DJ, Birtle AJ, Roberts IT. Triphasic locomotor response of a plant-parasitic nematode to avermectin: inhibition by the GABA antagonists bicuculline and picrotoxin. Parasitology. 1984, 88: 375-82.

79. Lupatini M, Suleiman A, Jacques R, Antoniolli Z, Ferreira A, Kuramae EE. et al. Network topology reveal high connectance levels and few key microbial genera within soils. Front Environ. Sci. 2014, 2: 1-11.

80. Yang J, Liang L, Li J, Zhang K. Nematicidal enzymes from microorganisms and their applications. Appl Microbiol Biotechnol. 2013, 97: 7081-95.

81. Aballay E, Prodan S, Zamorano A, Castaneda-Alvarez C. Nematicidal effect of rhizobacteria on plant-parasitic nematodes associated with vineyards. World J Microbiol Biotechnol. 2017, 33: 131.

82. Lenz KA, Miller TR, Ma H. Anabaenopeptins and cyanopeptolins induce systemic toxicity effects in a model organism the nematode Caenorhabditis elegans. Chemosphere. 2019, 214: 60-69.

83. Wenderoth M, Garganese F, Schmidt-Heydt M, Soukup ST, Ippolito A, Sanzani SM, Fischer R. Alternariol as virulence and colonization factor of Alternaria alternata during plant infection. Mol Microbiol. 2019, 112: 131-146.

84. Caboni P, Aissani N, Demurtas M, Ntalli N, Onnis V. Nematicidal activity of acetophenones and chalcones against Meloidogyne incognita and structure-activity considerations. Pest Manag Sci. 2016, 72: 125-30.

85. Yi C, Chen J, Wei C, Wu S, Wang S, Hu D, Song B. $\alpha$-Haloacetophenone and analogues as potential antibacterial agents and nematicides. Bioorg Med Chem Lett. 2020, 30: 126814.

86. Liu KL, He L, Wang JJ, Zhao ZM. Advances in the research on the pest resistance to avermectins. Chin Bull Entomol. 2007, 44:194-200.

87. Ghosh R, Andersen EC, Shapiro JA, Gerke JP, Kruglyak L. Natural variation in a chloride channel subunit confers avermectin resistance in C. elegans. Science. 2012, 335: $574-8$. 
88. Api AM, Belsito D, Botelho D, Bruze M, Burton Jr GA, Buschmann J, et al. RIFM fragrance ingredient safety assessment, acetophenone, CAS Registry Number 98-86-2. Food Chem Toxicol. 2018, 118 Suppl 1: S162-S169.

89. Franco TA, Xu P, Brito NF, Oliveira DS, Wen X, Moreira MF, Unelius CR, Leal WS, Melo ACA. Reverse chemical ecology-based approach leading to the accidental discovery of repellents for Rhodnius prolixus, a vector of Chagas diseases refractory to DEET. Insect Biochem Mol Biol. 2018, 103: 46-52.

90. Erbilgin N, Gillette NE, Mori SR, Stein JD, Owen DR, Wood DL. Acetophenone as an anti-attractant for the western pine beetle, Dendroctonus Brevicomis LeConte (Coleoptera: Scolytidae). J Chem Ecol. 2007, 33: 817-23.

91. Santhanam R, Menezes RC, Grabe V, Li D, Baldwin IT, Groten K. A suite of complementary biocontrol traits allows a native consortium of root-associated bacteria to protect their host plant from a fungal sudden-wilt disease. Mol Ecol. 2019, 28: 1154-1169.

\section{Figure legends}

Figure 1 Taxonomic network inference of the rhizosphere microbiota. (A) and (B): Co-occurrence network based on correlation of 16S rRNA reads (species level) extracted from metagenomes of rhizosphere soil in the two conditions: non-infected soil (NS), and root-knot infected soil (RNI), respectively. (C) and (D): Co-occurrence network based on correlation of $18 \mathrm{~S}$ rRNA reads (species level) extracted from metagenomes of the NS and RNI rhizosphere microbiota, respectively. The size of each node is proportional to the number of connections (node degree). Nodes belonging to different phylum are differently colored. Blue lines and red lines indicate negative and positive interactions between different nodes, respectively. Modules with more than five nodes are included.

Figure $2 \mathrm{Zi}-\mathrm{Pi}$ plot indicates the distribution of nodes based on their topological roles in bacterial network (A) and fungal network (B). Each symbol represents a node in the non-infected soil (NS) network (red triangle) or root-knot infected soil (RNI) network (blue square). The threshold values of $\mathrm{Zi}$ and $\mathrm{Pi}$ for categorizing nodes are 2.5 and 0.62 , respectively.

Figure 3 Function network inference of the rhizosphere microbiota. (A) and (B): Co-occurrence network based on correlation of COG genes in the two conditions: non-infected soils (NS), and root-knot infected soils (RNI), respectively. The size of each node is 
proportional to the abundance of nodes. Nodes belonging to different category are differently colored. Blue lines and red lines indicate negative and positive interactions between different nodes, respectively. Node shapes are based on change of node abundance: circle, non-change nodes in any treatment; triangle, overrepresented in the NS condition; diamond, overrepresented in the RNI condition. (C) and (D): Co-occurrence network based on correlation of BGCs genes in the NS and RNI microbiota. Nodes are shaped by BGC types and the size is correlated with the abundance of each node. Node color is based on change of node abundance. Yellow, non-change nodes in any treatment; red: enriched in the RNI microbiota, green: enriched in the NS microbiota.

Figure 4 Metabolomics analysis of microbiota in the NS and RNI rhizosphere soils. (A) and (B): PLS-DA analysis in negative polarity mode and positive polarity mode, respectively. (C) and (D): Different metabolites in negative and positive polarity mode, respectively. (E) and (F): Z-score of different metabolites in negative and positive polarity mode, respectively.

Figure 5 Interactions between acetophenone and different microbes. Nodes of microbes are indicated with green color (except the two nodes interacting with acetophone are represented with yellow color), and node of acetophenone is indicated with yellow color. Blue lines and red lines indicate negative and positive interactions between two nodes, respectively.

Figure S1 Overview of annotated genes in the metagenome after quality filtering. (A) Percentage of reads taxonomically classified at Domain level. (B) Percentage of annotated reads using the KEGG database. (C) Percentage of annotated reads using the eggNOG database.

Figure S2 Overview and distribution of microbial reads extracted from the metagenome. (A) Beta diversity of microbial community in root-knot nematode infected soil (RNI) and non-infected soil (NS). Unigenes were used to calculate Bray-Curtis distances and conduct Principal Co-ordinates Analysis (87.1\% of the overall variance). Significance of the PCoA analysis was assessed through Anosim $(P<0.01)$. (B-D) Histograms showing the taxonomic distribution at Phylum, Class and Family level, respectively.

Figure S3 Microbes with potential nematicidal activity are enriched in the non-infected soils. (A) Comparing abundance of microbes between the NS and RNI soils. (B) Correlation of enriched bacteria with soil properties. 
Figure S4 Nematicidal bacteria isolated from the NS rhizosphere soils. (A) Nematicidal activity of bacteria isolates. (B) Phylogenetic tree of the bacterial isolates. Colors depict the taxonomic classification. Green bar charts represent the nematicidal activity of each isolate. (C) Phylogenetic tree of the fungal isolates.

Figure S5 Relative abundances of different nodes in NS and RNI networks. (A) Proportion of nodes belonging to different phylogenetic groups in the NS and RNI bacterial networks; Venn diagrams indicate the number of nodes shared and not shared by the NS and RNI bacterial networks. (B) Proportion of nodes belonging to different phylogenetic groups in the NS and RNI fungal networks; Venn diagrams indicate the number of nodes shared and not shared by the NS and RNI fungal networks.

Figure S6 Sub-network of nodes belonging to Proteobacteria (A), or Ascomycota and Basidiomycota (B). The circle sizes represent the mean read relative abundance of the differentially abundant taxa. Bacterial or fungal species that are significantly enriched in the comparison between NS and RNI are indicated in green for NS and in blue for RNI; nonsignificant taxa are indicated in yellow. Red color lines represent positive interaction. Blue color lines represent negative interaction.

Figure S7 Heatmaps depict relative abundance of the significantly enriched genes involved in polyketide biosynthesis (A), CAZymes (B) or enzymes $(C, D)$ in the NS microbiota as compared to the RNI microbiota.

Figure S8 The protease, chitinase and lipase activities of nematicidal bacteria. The enzymic activity was represented by the ratio of clearing zone diameter to the bacterial and fungal colony diameter.

Figure S9 The abundances of BGCs in two groups. Comparing BGCs gene abundance between the NS and RNI microbiota.

Figure S10 Comparing gene abundances between the NS and RNI BGCs network.

Figure S11 Metabolomics analysis of rhizosphere microbiota in the NS and RNI soils. (A),

(B): PCA analysis in negative polarity mode and positive polarity mode, respectively.

Figure S12 The hierarchical clustering of different metabolites in negative polarity mode (A) and positive polarity mode (B), respectively. 
Figure S13 Nematicidal activity of metabolites and microbes. (A) Nematicidal activity of different concentrations of acetophenone against M. incognita. (B) Avoidance of acetophenone against $M$. incognita. (C) Nematicidal activity of B. amyloliquefaciens W1, B. butanolivorans N13, B. drentensis A14, B. marisflavi L4, B. wiedmannii L5, B. tequilensis T9, respectively. (D) Phenylalanine metabolism pathway from KEGG.

Table S1 Properties of non-infected (NS) and root-knot nematode infected soils (RNI)

Table S2 Correlation of soil properties and number of Meloidogyne incognita

Table S3 Metagenome sequence statistics after filtering

Table S4 CDS statistics of each sample

Table S5 Correlations and topological properties of the co-occurrence fungal network analysis

Table S6 Correlations and topological properties of the co-occurrence bacterial network analysis

Table S7 Correlations and topological properties of the co-occurrence functional network analysis

Table S8 Correlation of metabolites and number of Meloidogyne incognita

Table S9 Metabolites in Bacillus amyloliquefaciens W1 culture 
Figure 1 Taxonomic network inference of the rhizosphere microbiota. (A) and (B): Co-occurrence network based on correlation of 16S rRNA reads (species level) extracted from metagenomes of rhizosphere soil in the two conditions: non-infected soil (NS), and root-knot infected soil (RNI), respectively. (C) and (D): Co-occurrence network based on correlation of 18S rRNA reads (species level) extracted from metagenomes of the NS and RNI rhizosphere microbiota, respectively. The size of each node is proportional to the number of connections (node degree). Nodes belonging to different phylum are differently colored. Blue lines and red lines indicate negative and positive interactions between different nodes, respectively. Modules with more than five nodes are included.

(A)

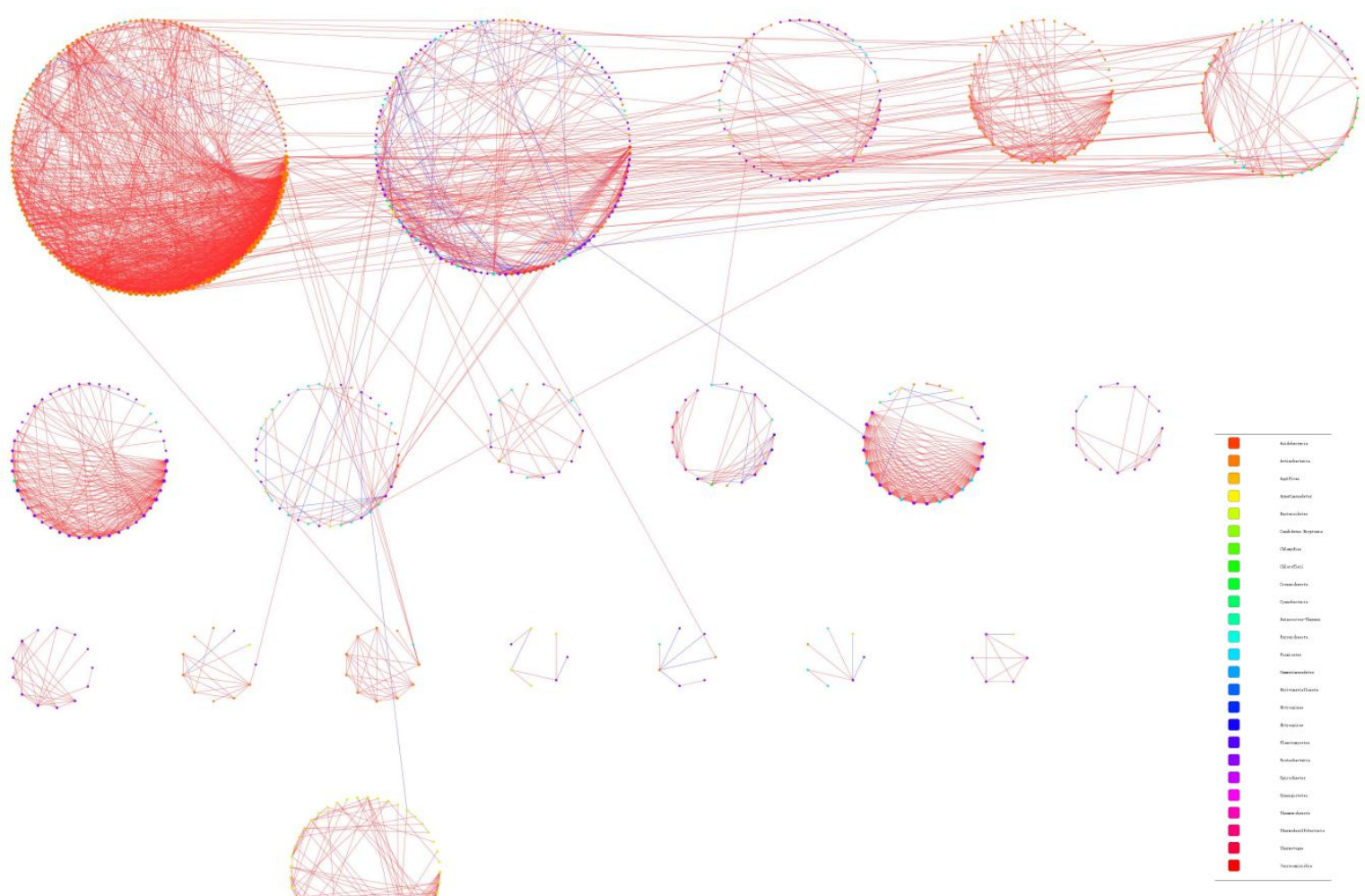


(B)

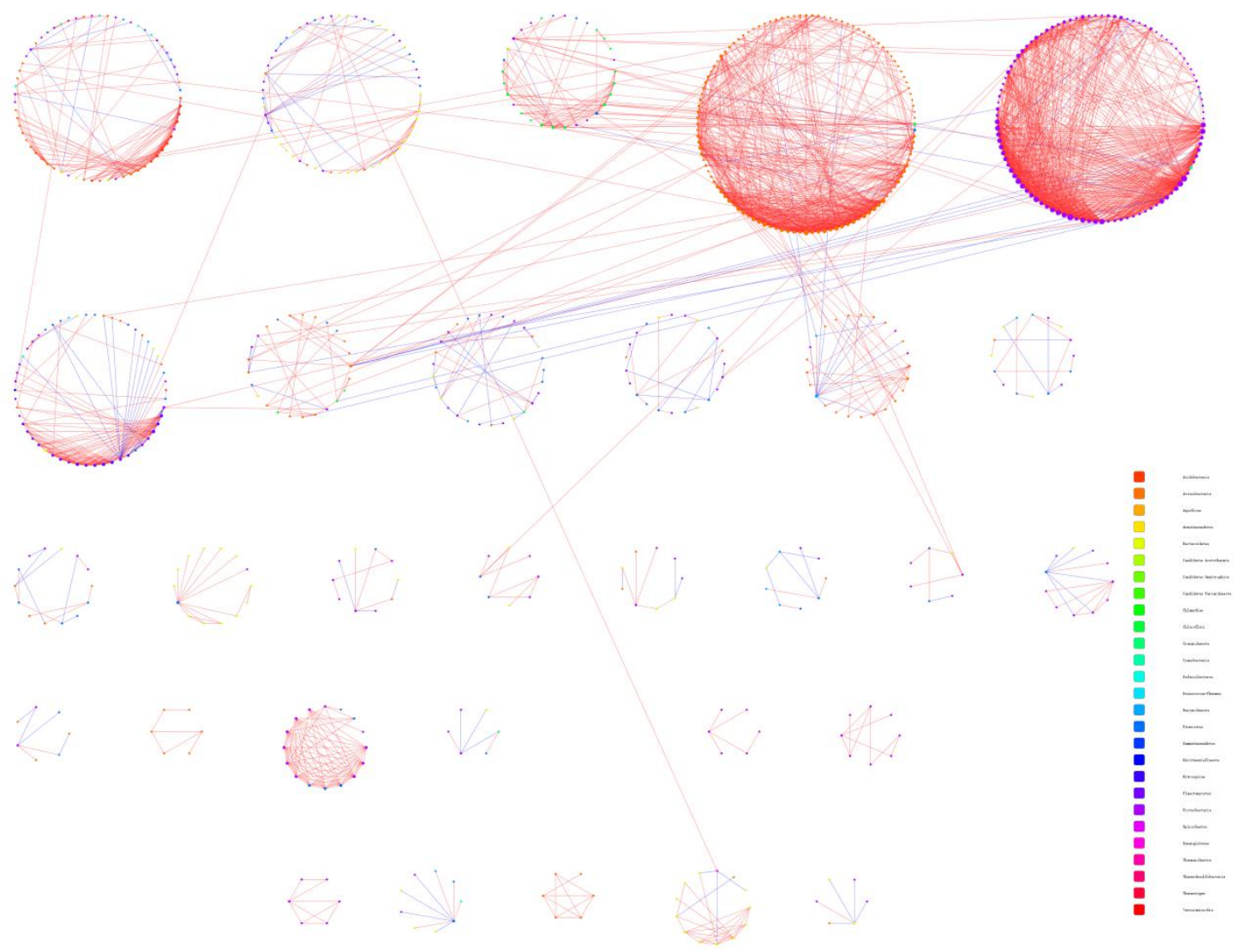

(C)

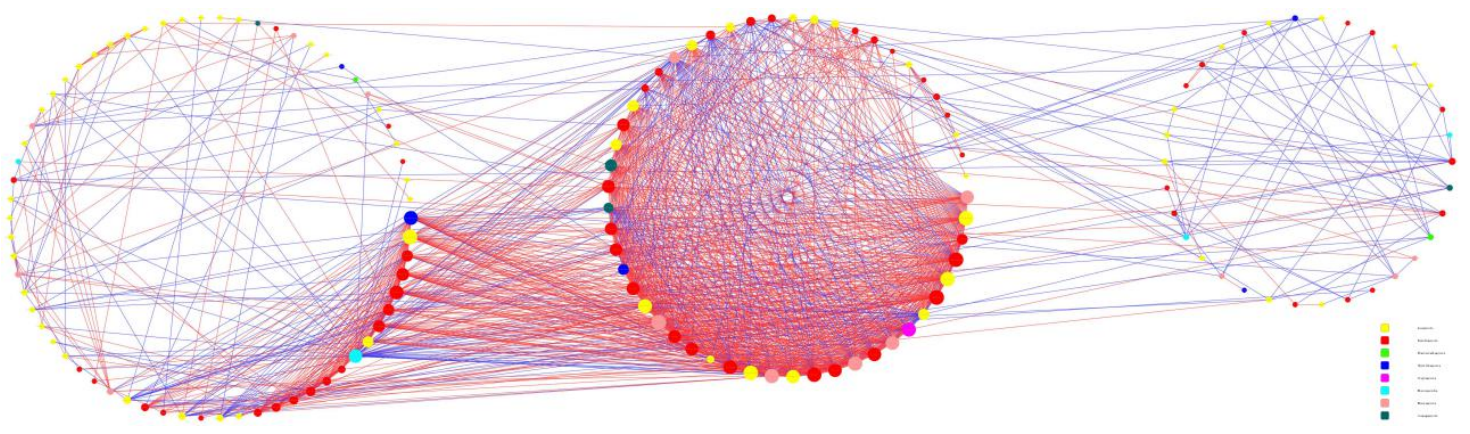

(D)

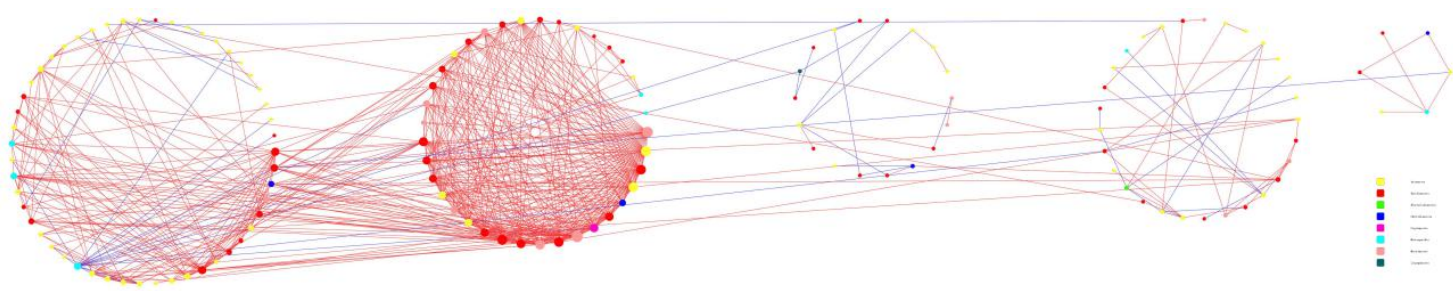


Figure $2 \mathrm{Zi}$-Pi plot indicates the distribution of nodes based on their topological roles in bacterial network (A) and fungal network (B). Each symbol represents a node in the non-infected soil (NS) network (red triangle) or root-knot infected soil (RNI) network (blue square). The threshold values of $\mathrm{Zi}$ and $\mathrm{Pi}$ for categorizing nodes are 2.5 and 0.62, respectively.

(A)

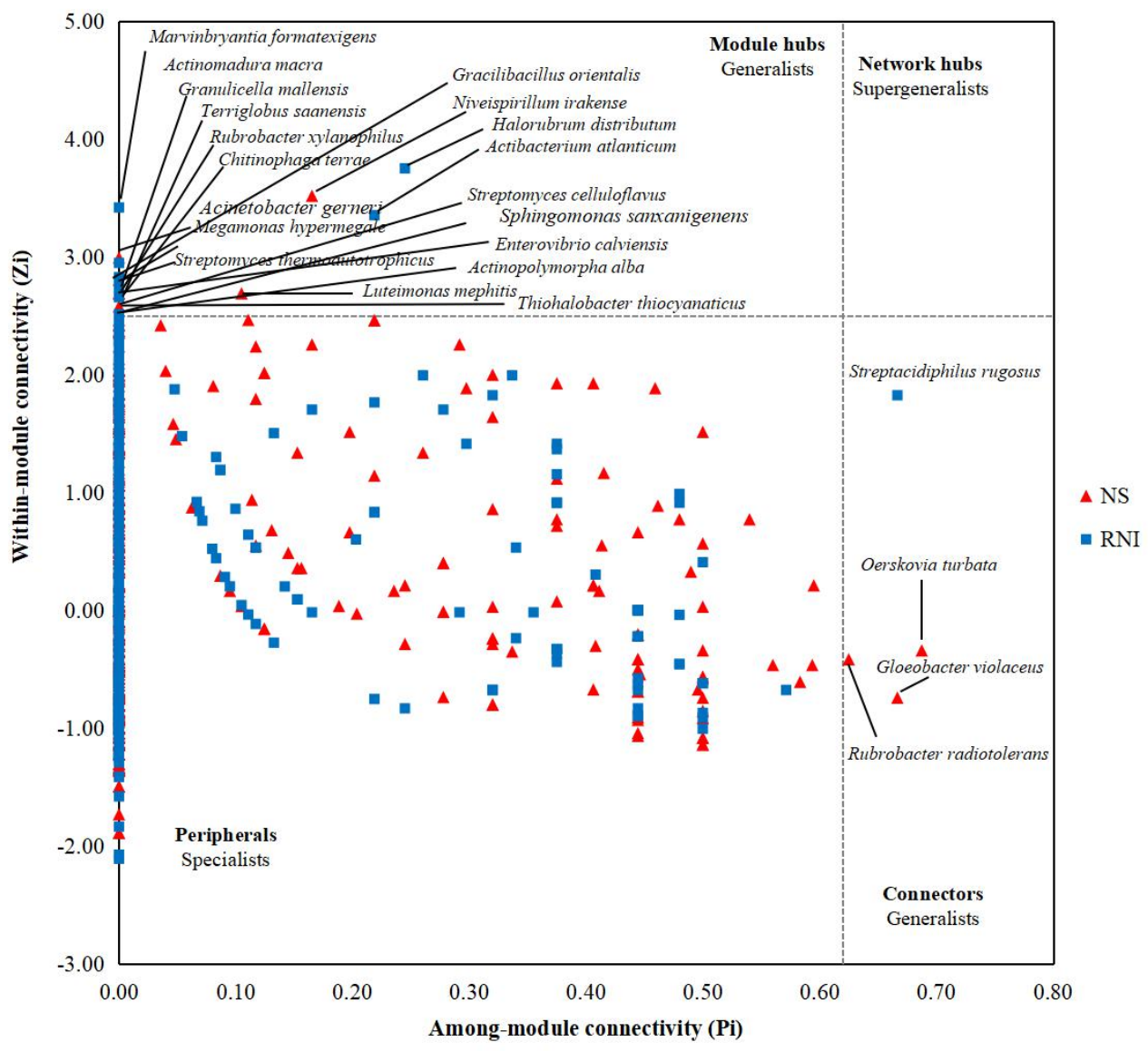


(B)

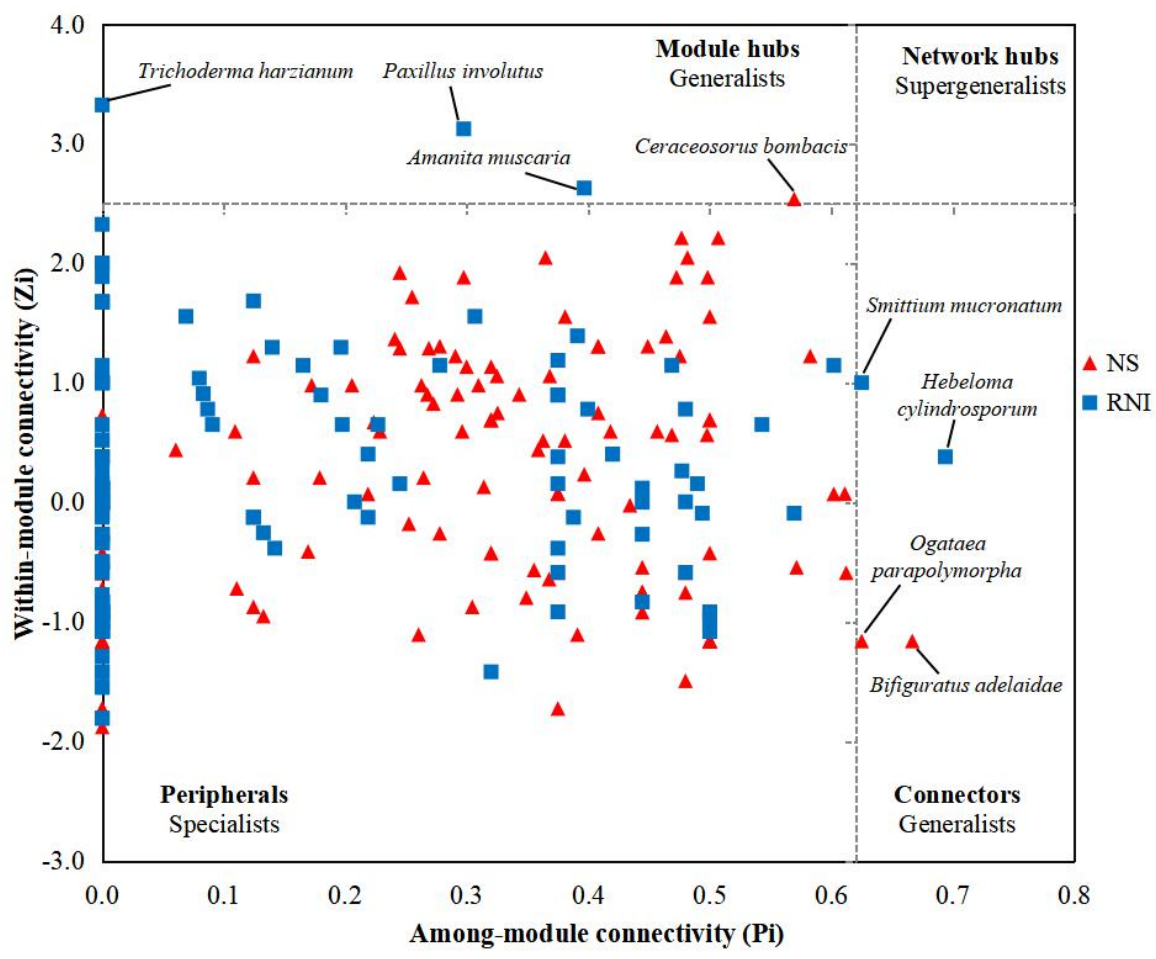


Figure 3 Function network inference of the rhizosphere microbiota. (A) and (B): Co-occurrence network based on correlation of COG genes in the two conditions: non-infected soils (NS), and root-knot infected soils (RNI), respectively. The size of each node is proportional to the abundance of nodes. Nodes belonging to different category are differently colored. Blue lines and red lines indicate negative and positive interactions between different nodes, respectively. Node shapes are based on change of node abundance: circle, non-change nodes in any treatment; triangle, overrepresented in the NS condition; diamond, overrepresented in the RNI condition. (C) and (D): Co-occurrence network based on correlation of BGCs genes in the NS and RNI microbiota. Nodes are shaped by BGC types and the size is correlated with the abundance of each node. Node color is based on change of node abundance. Yellow, non-change nodes in any treatment; red: enriched in the RNI microbiota, green: enriched in the NS microbiota.

(A)

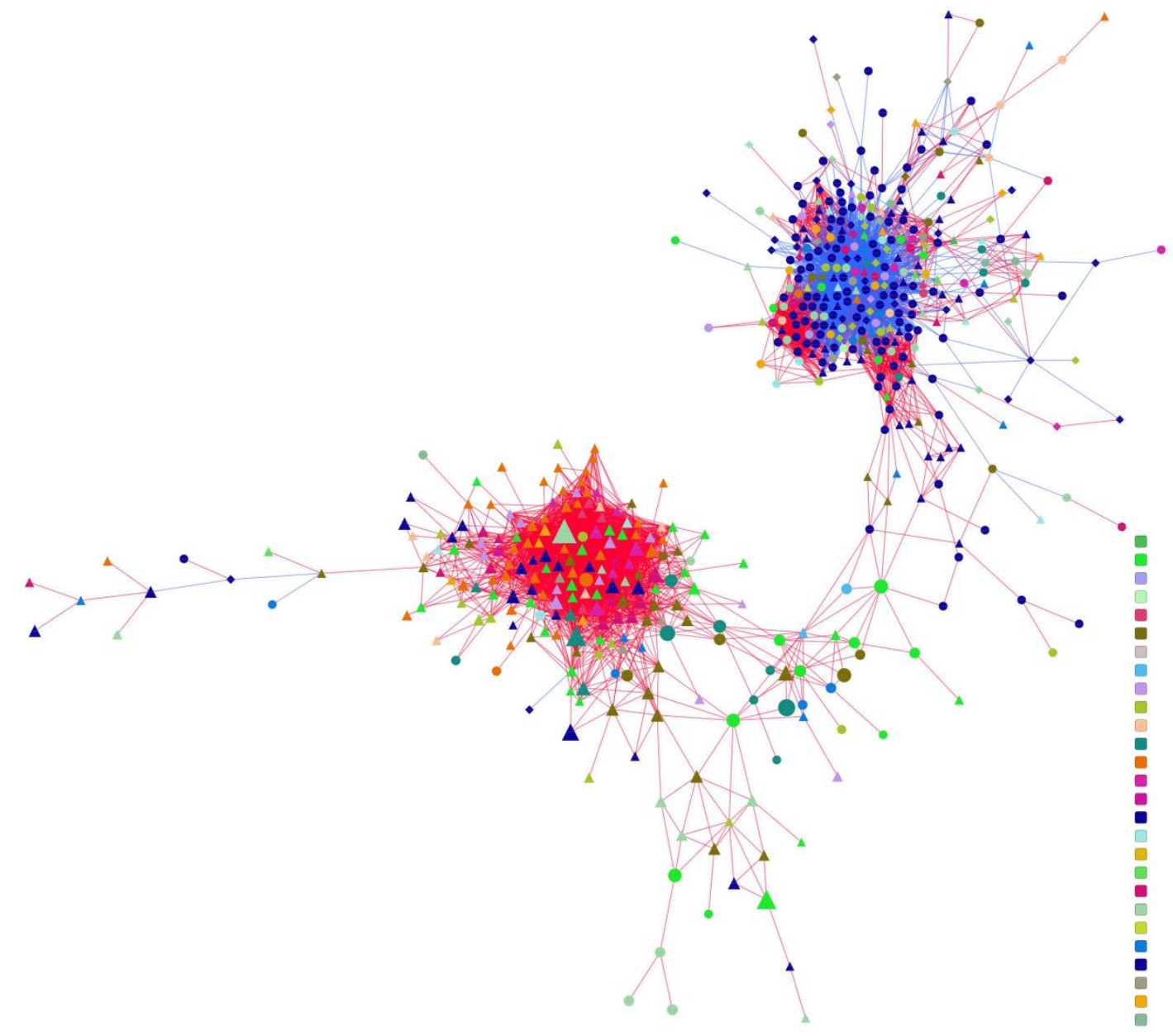


(B)

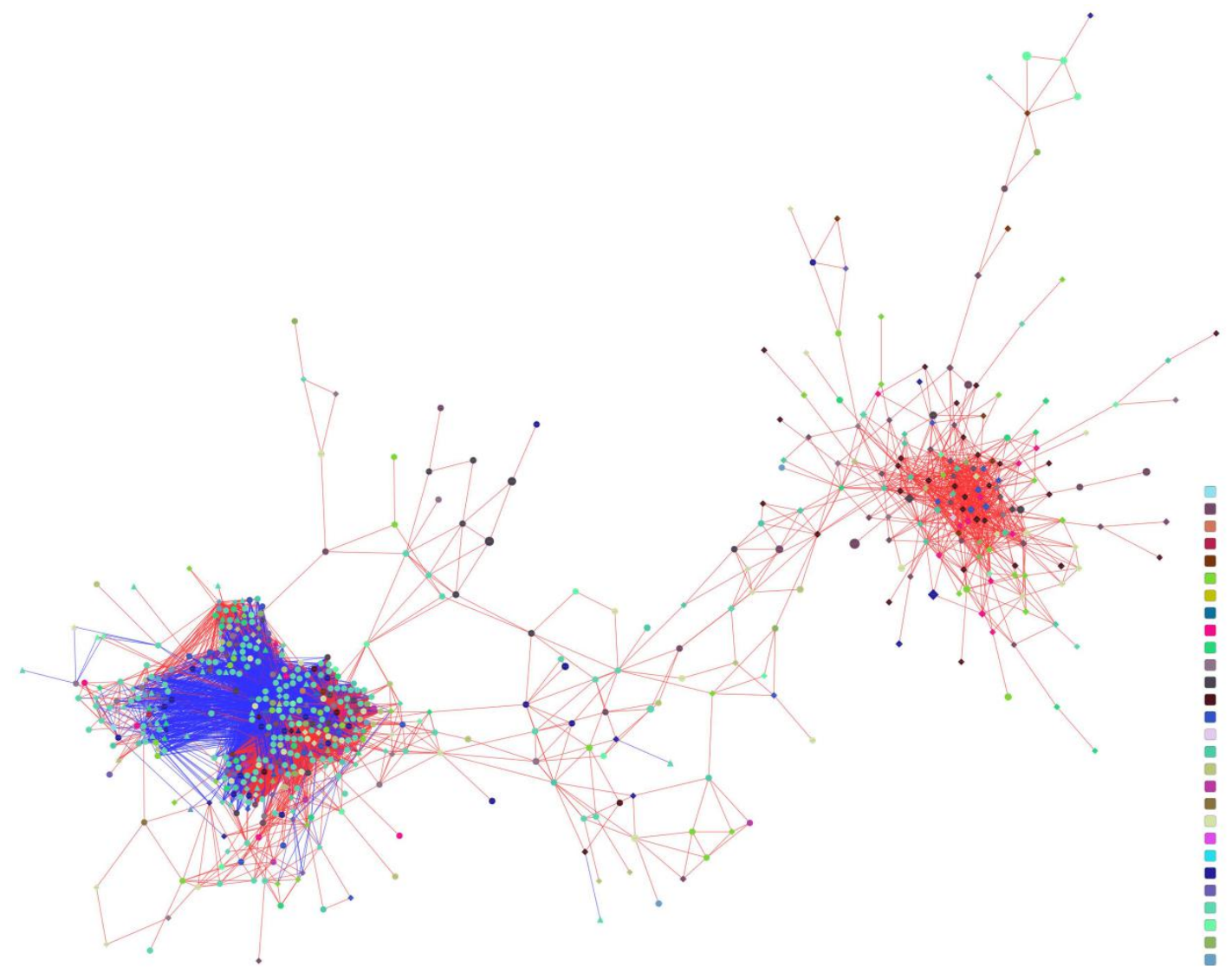


(C)

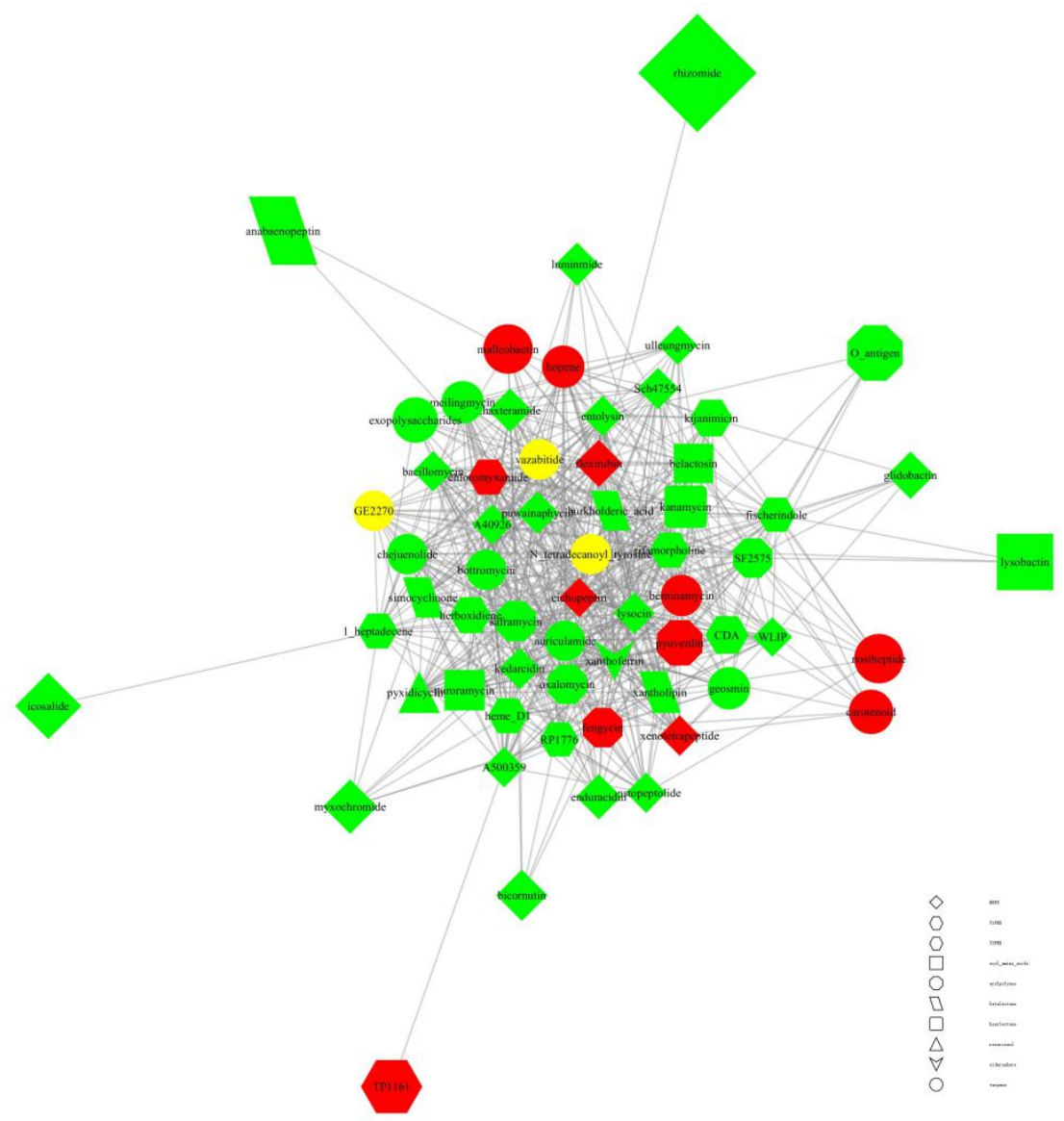

(D)

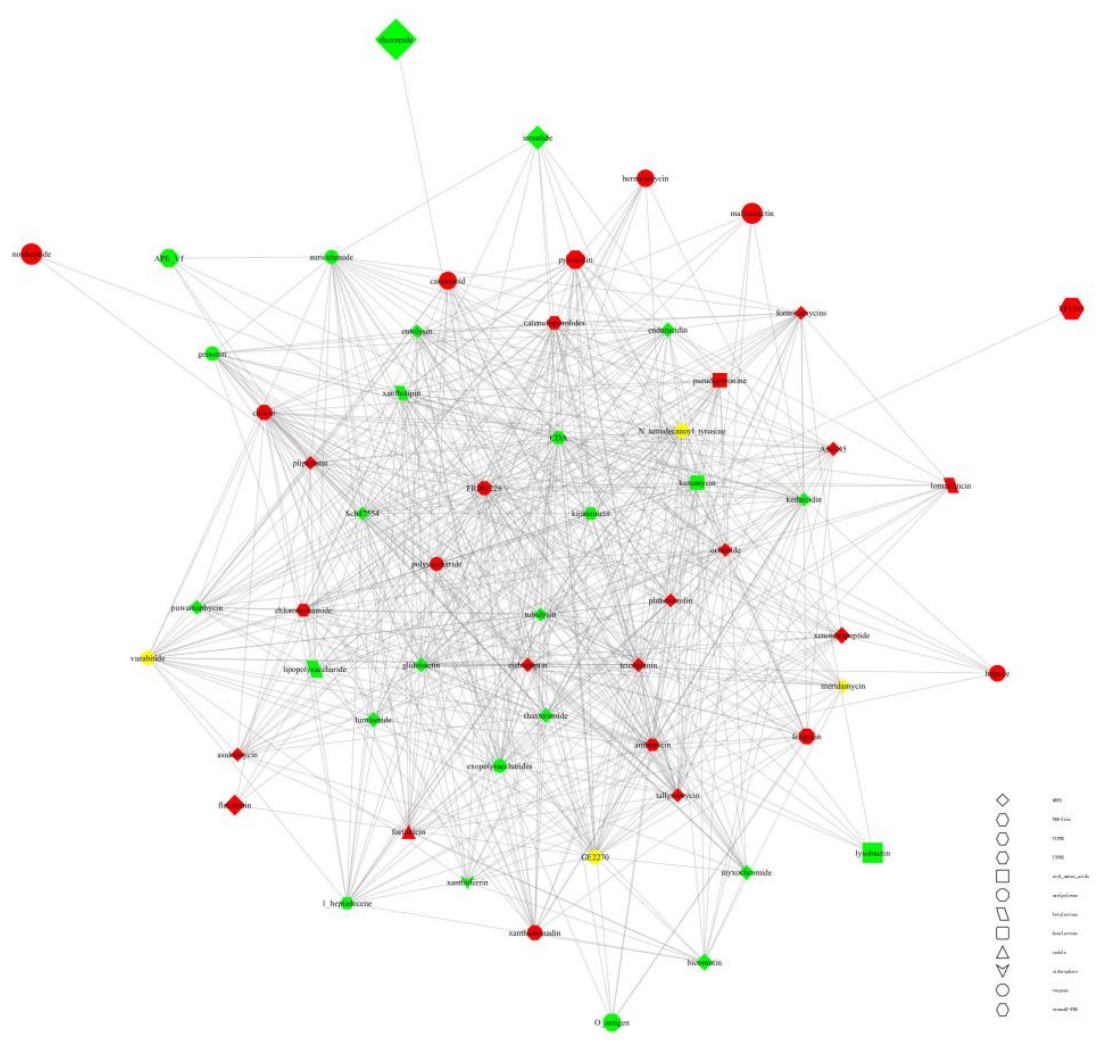


Figure 4 Metabolomics analysis of microbiota in the NS and RNI rhizosphere soils. (A) and (B): PLS-DA analysis in negative polarity mode and positive polarity mode, respectively. (C) and (D): Different metabolites in negative and positive polarity mode, respectively. (E) and $(\mathbf{F})$ : Z-score of different metabolites in negative and positive polarity mode, respectively.

(A)

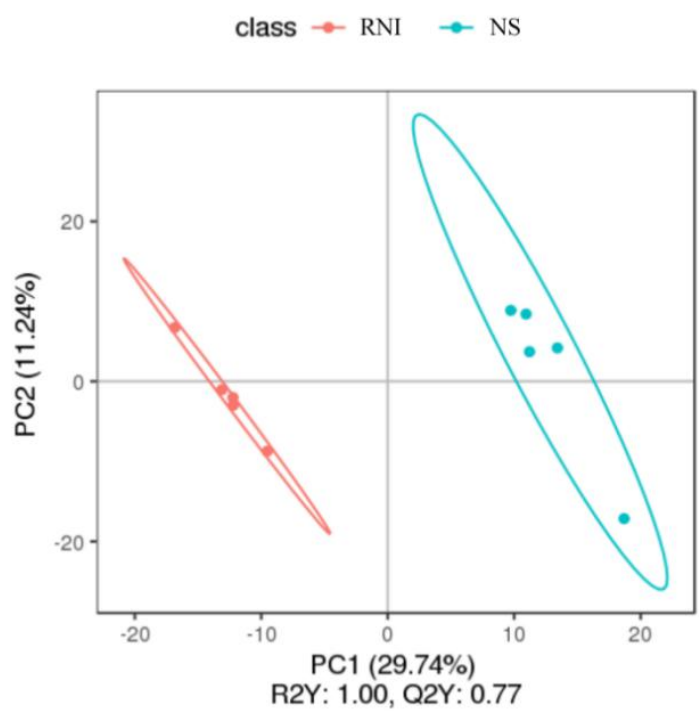

(C)

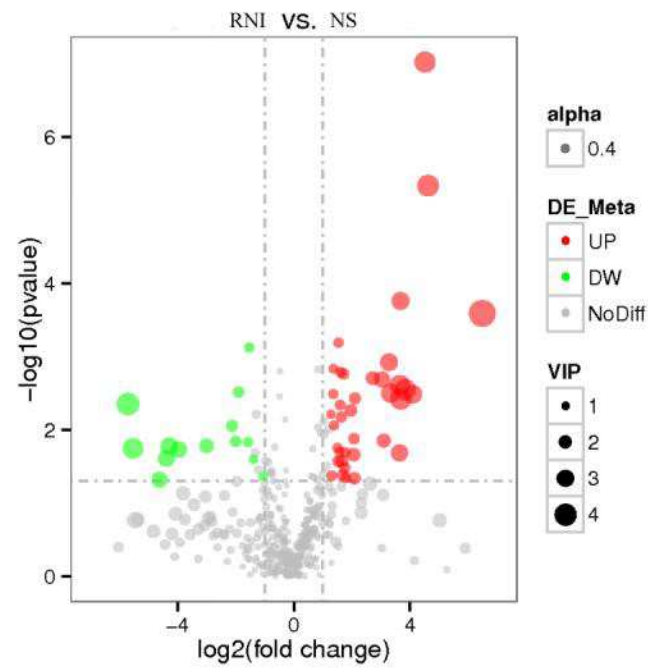

(B)

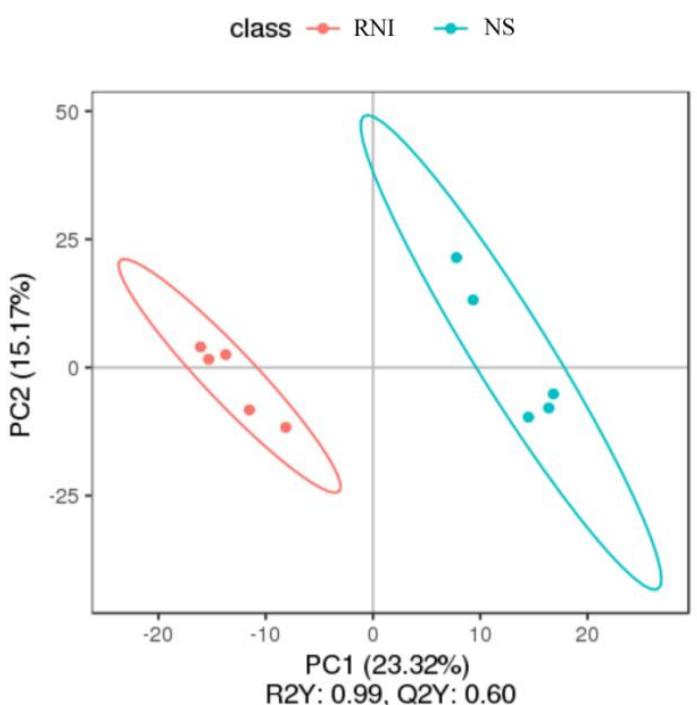

(D)

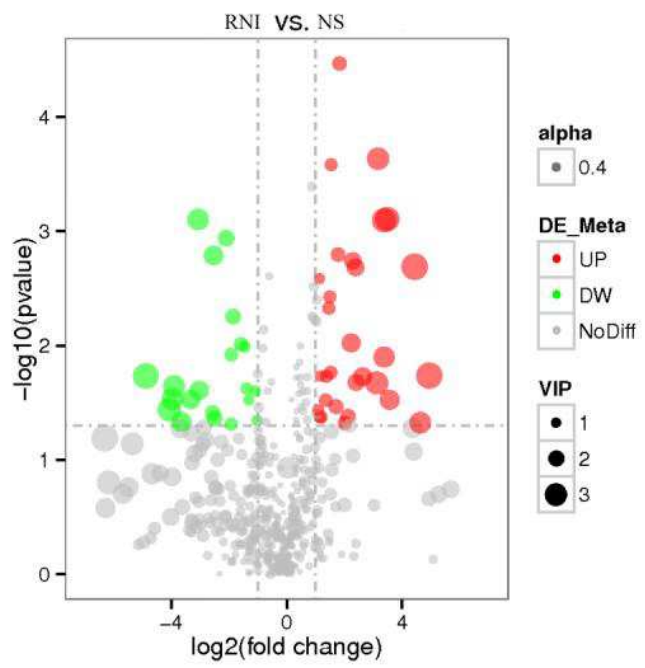


(E)

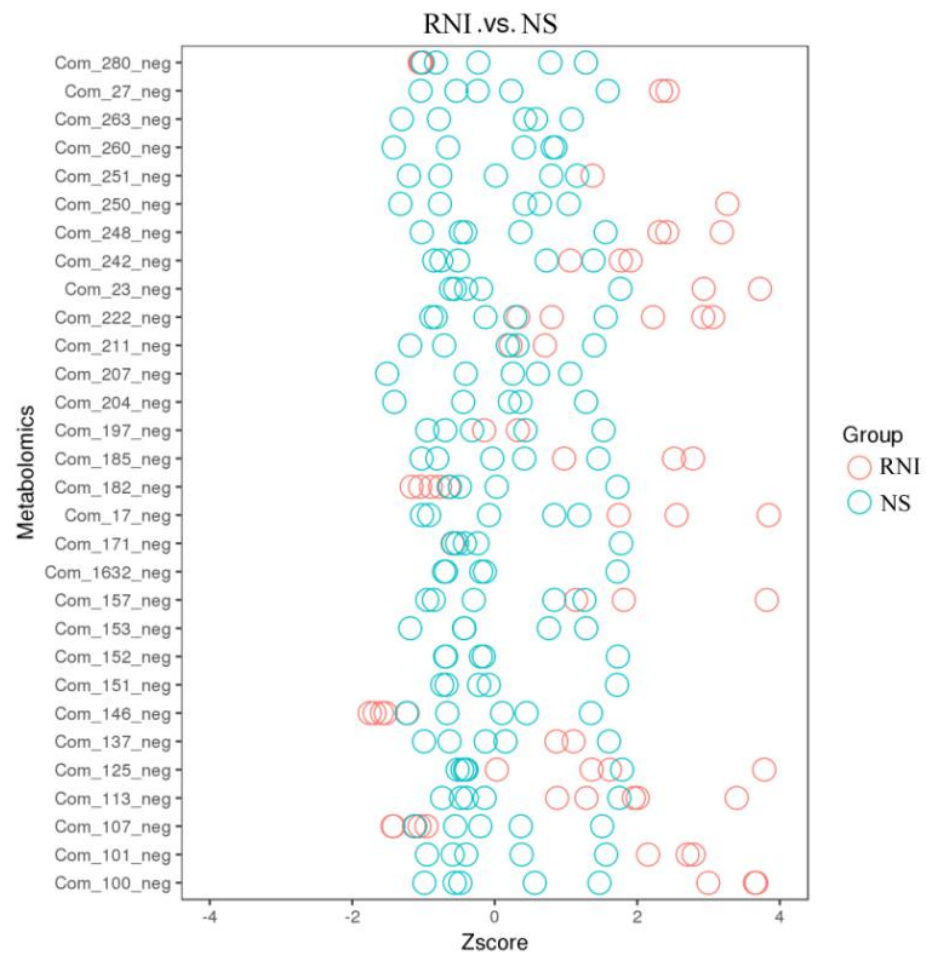

(F)

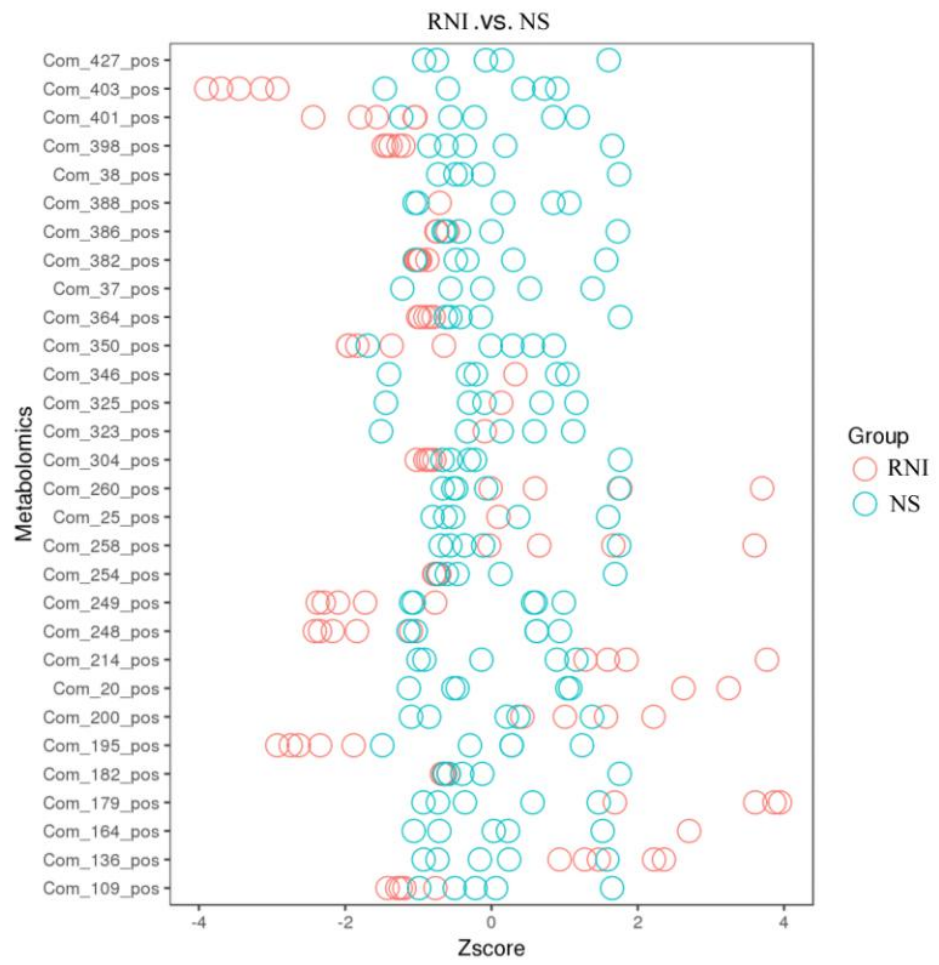


Figure 5 Interactions between acetophenone and different microbes. Nodes of microbes are indicated with green color (except the two nodes interacting with acetophone are represented with yellow color), and node of acetophenone is indicated with yellow color. Blue lines and red lines indicate negative and positive interactions between two nodes, respectively.

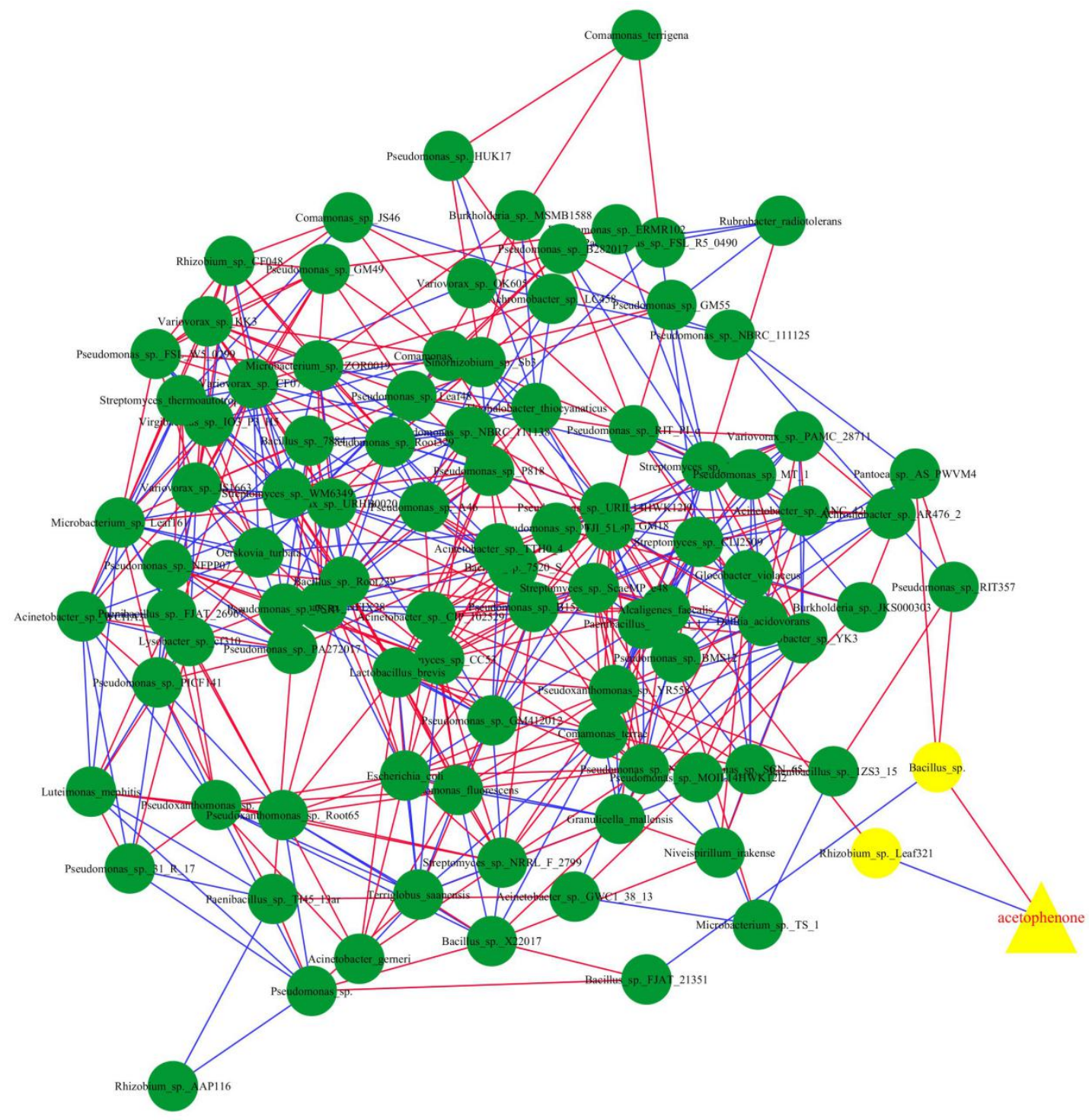


$\mathbf{A}$

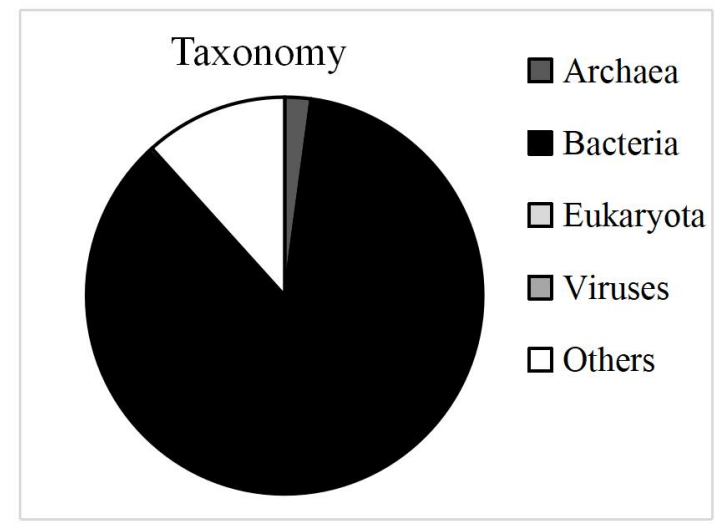

B

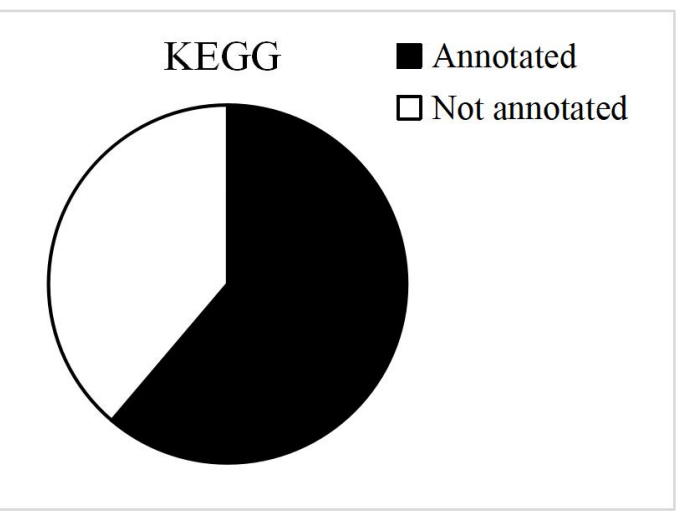

C

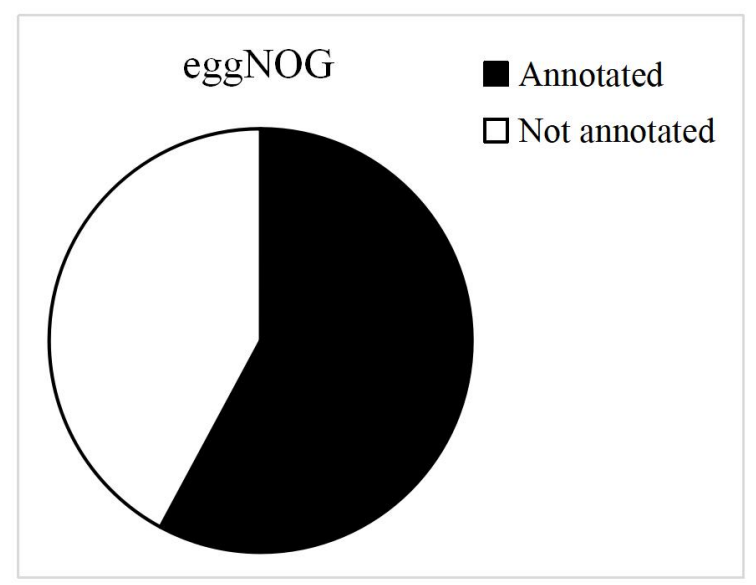

Figure S1 Overview of annotated genes in the metagenome after quality filtering. (A) Percentage of reads taxonomically classified at Domain level. (B) Percentage of annotated reads using the KEGG database. (C) Percentage of annotated reads using the eggNOG database. 
A

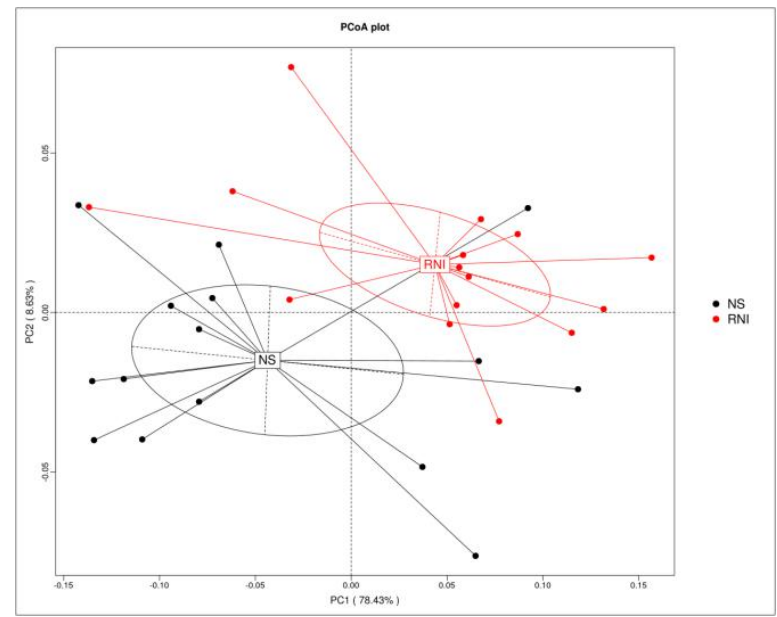

B

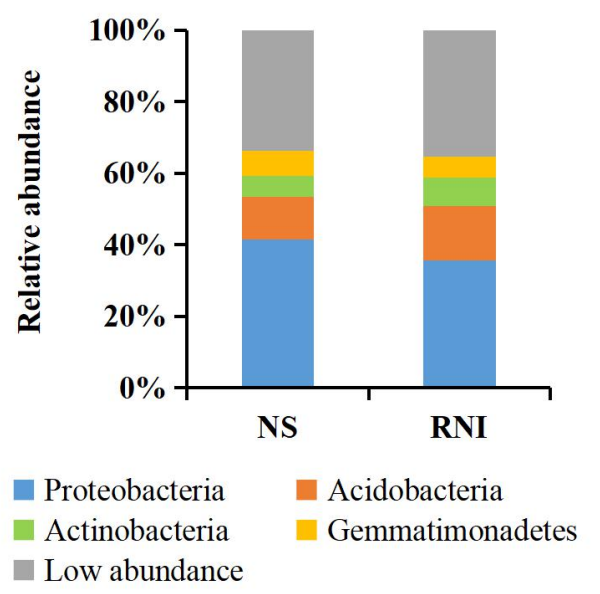

C

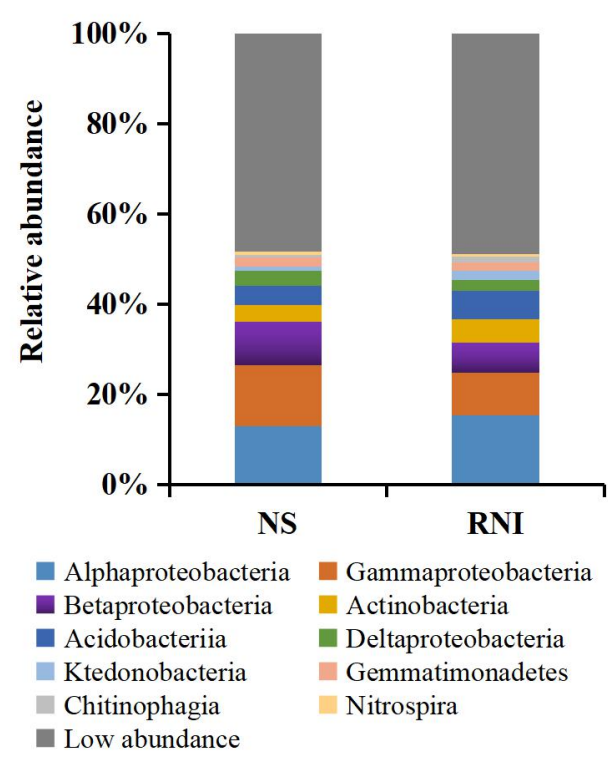

D

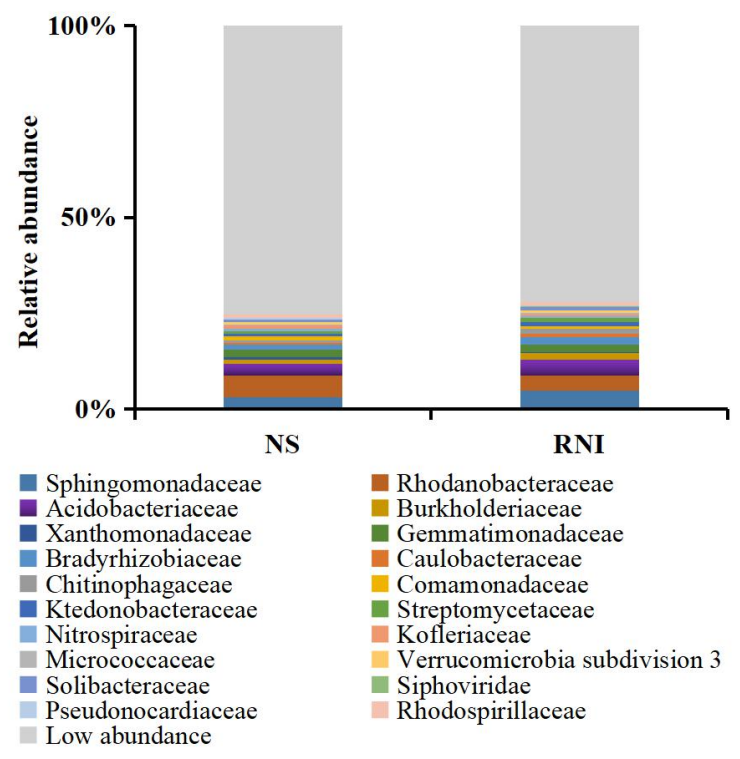

Figure S2 Overview and distribution of microbial reads extracted from the metagenome.

(A) Beta diversity of microbial community in root-knot nematode infected soil (RNI) and non-infected soil (NS). Unigenes were used to calculate Bray-Curtis distances and conduct Principal Co-ordinates Analysis (87.1\% of the overall variance). Significance of the PCoA analysis was assessed through Anosim $(P<0.01)$. (B-D) Histograms showing the taxonomic distribution at Phylum, Class and Family level, respectively. 
A
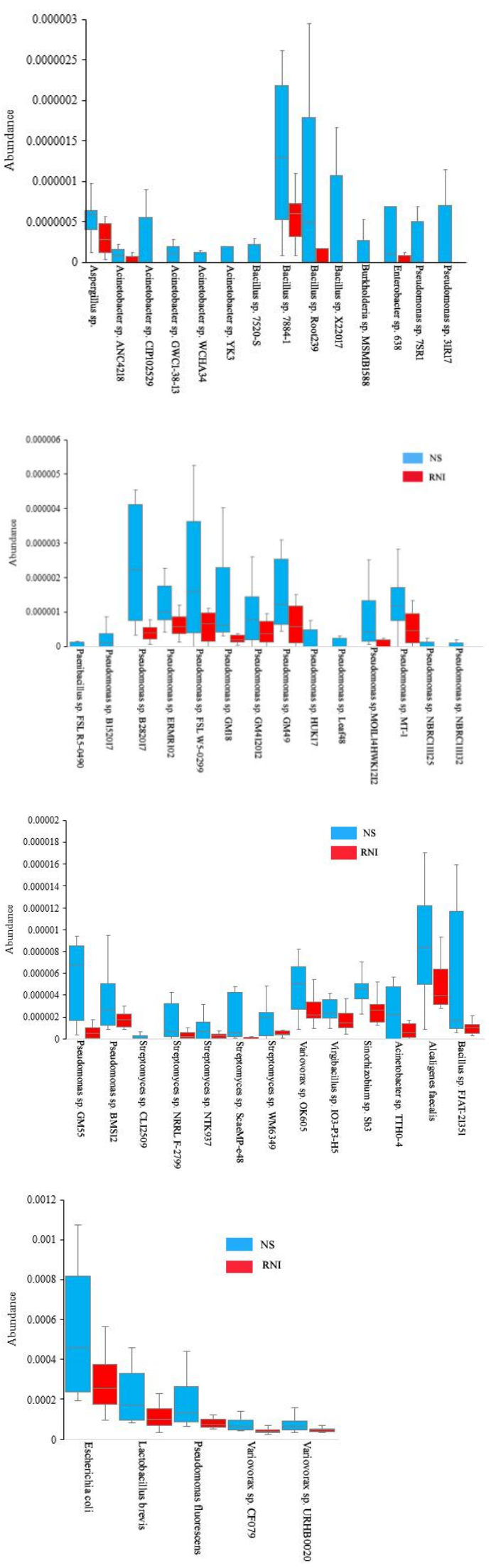
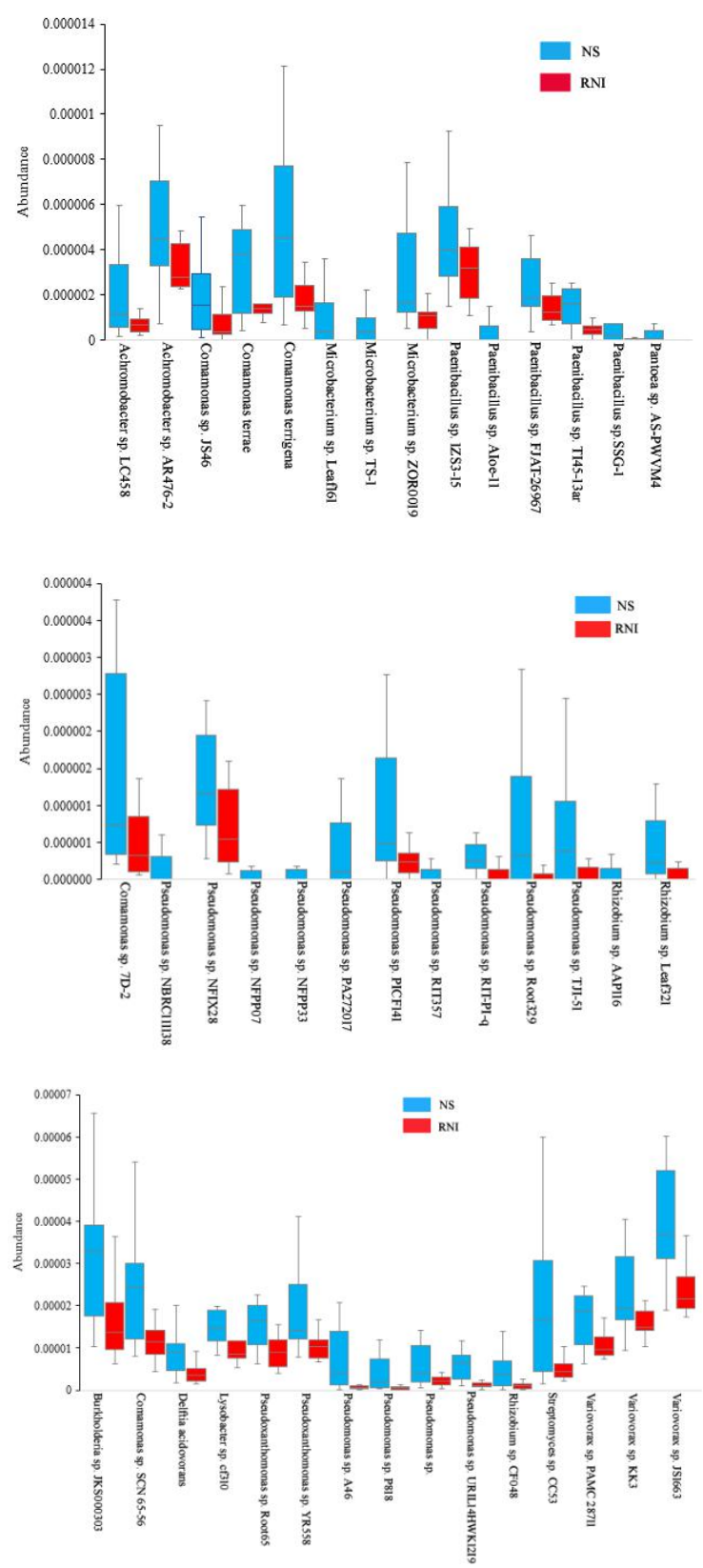
B
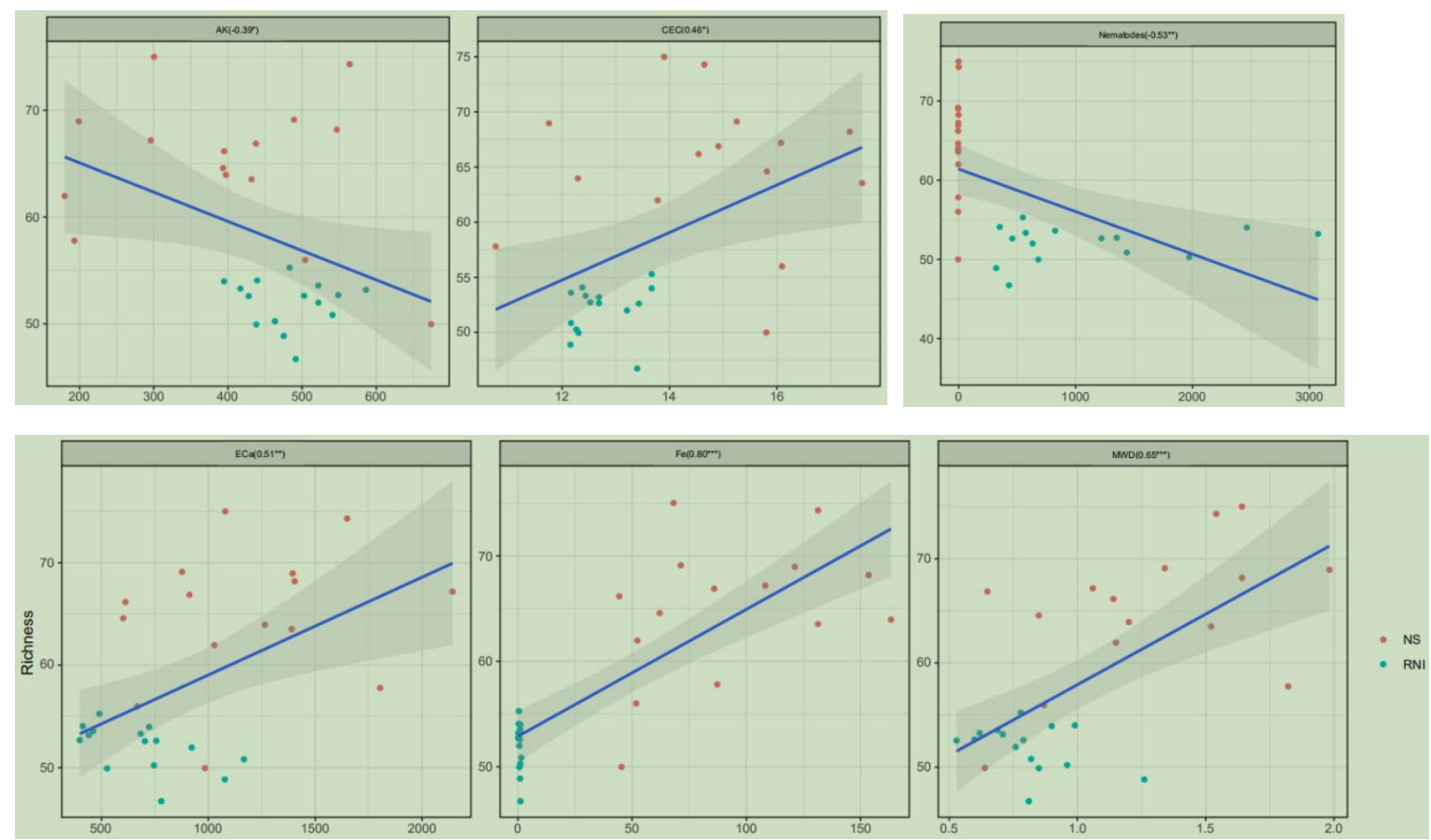

Figure S3 Microbes with potential nematicidal activity are enriched in the non-infected soils. (A) Comparing abundance of microbes between the NS and RNI soils. (B) Correlation of enriched bacteria with soil properties. 
A

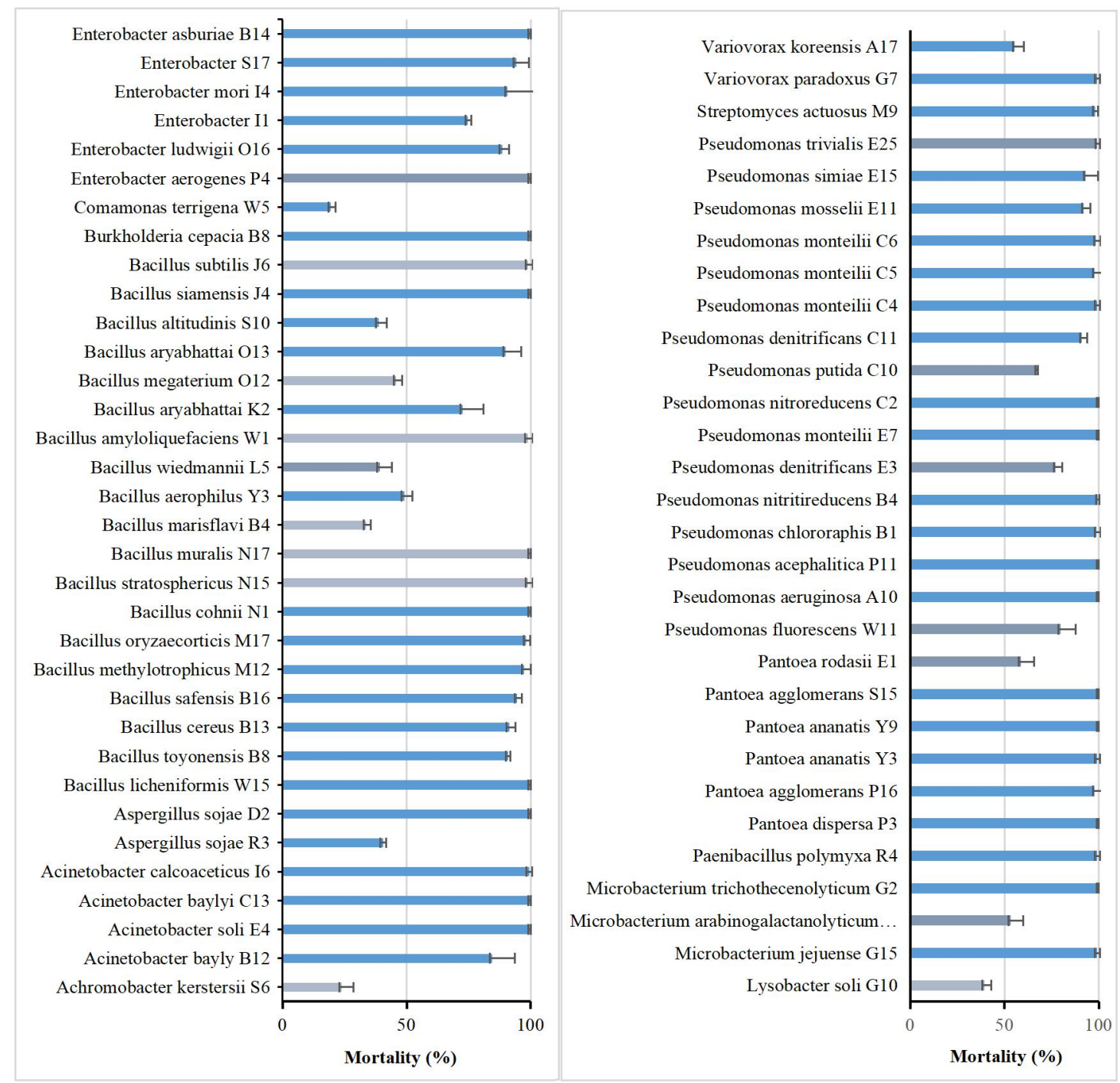

B

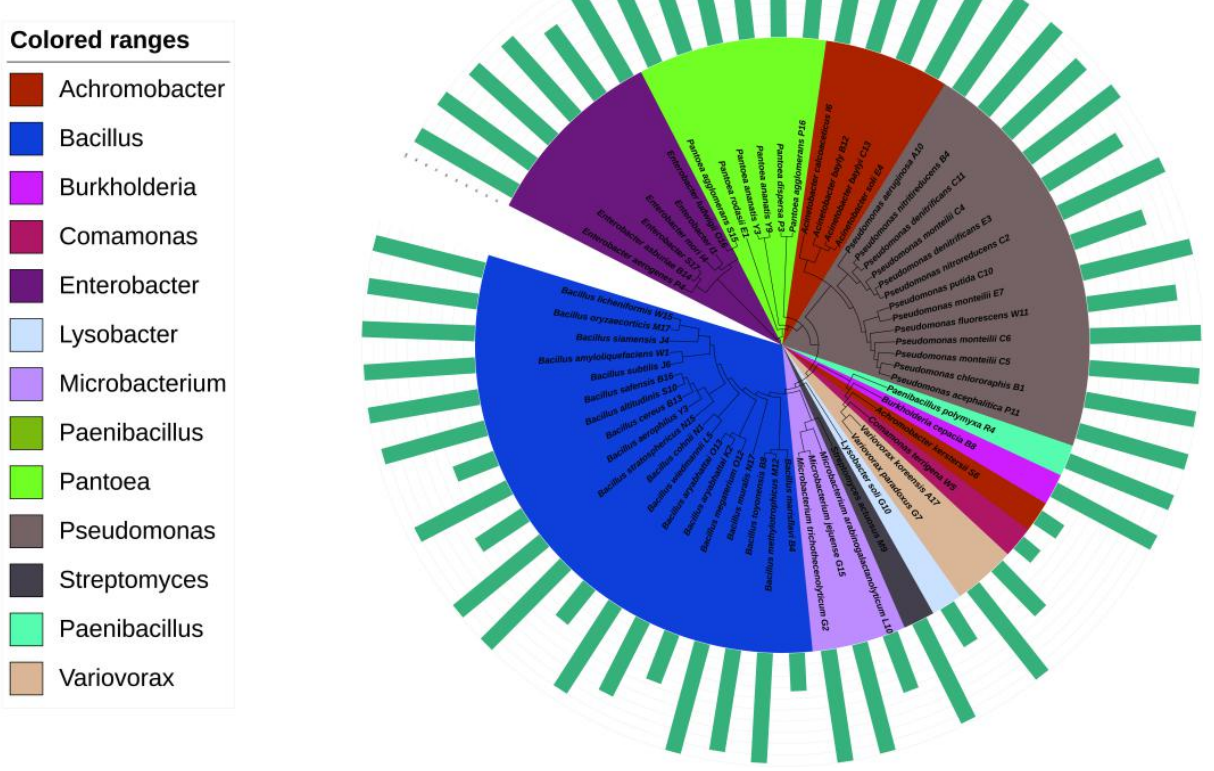


C

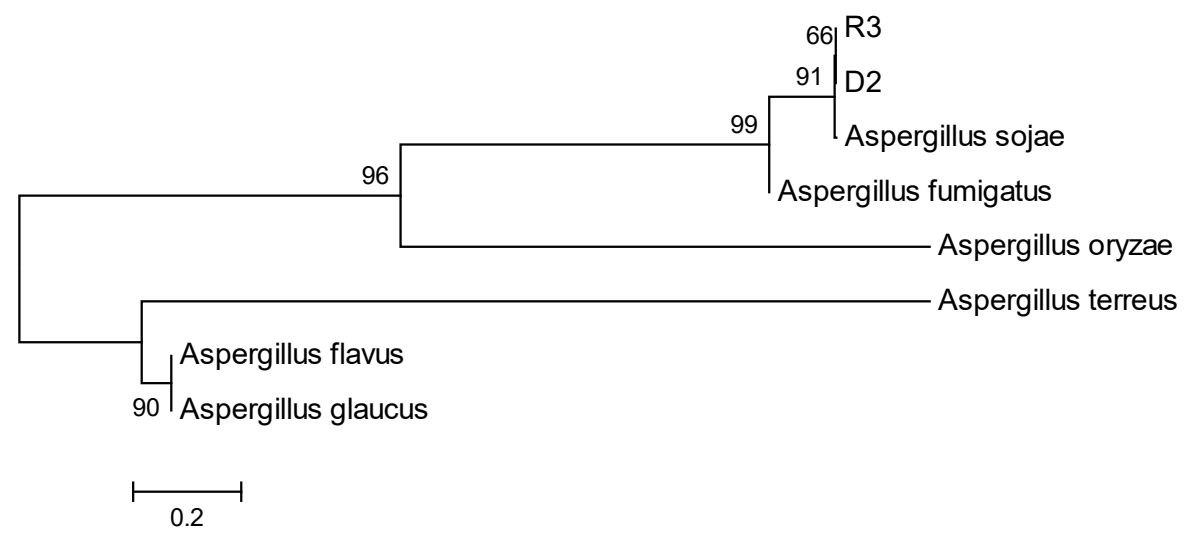

Figure S4 Nematicidal bacteria isolated from the NS rhizosphere soils. (A) Nematicidal activity of bacteria isolates. (B) Phylogenetic tree of the bacterial isolates. Colors depict the taxonomic classification. Green bar charts represent the nematicidal activity of each isolate. (C) Phylogenetic tree of the fungal isolates. 
$\mathbf{A}$

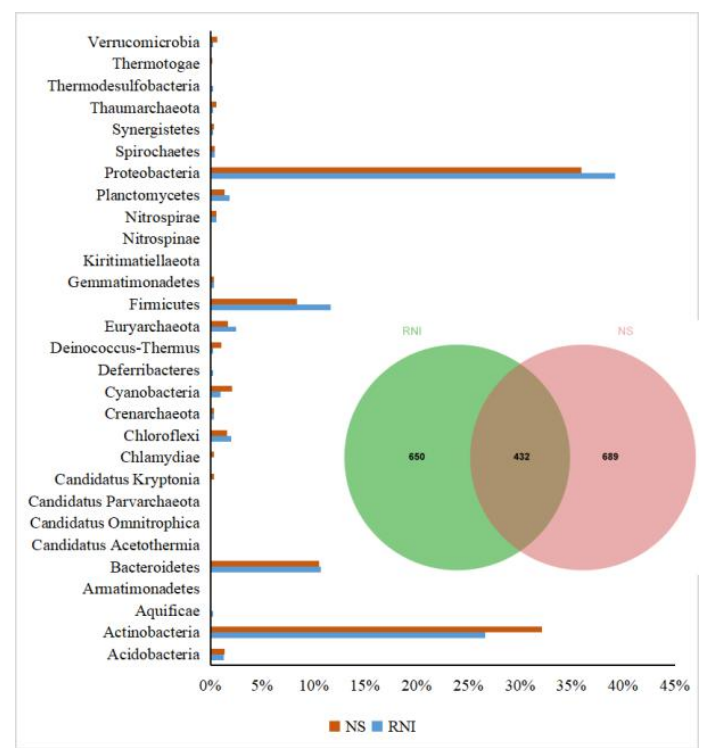

B

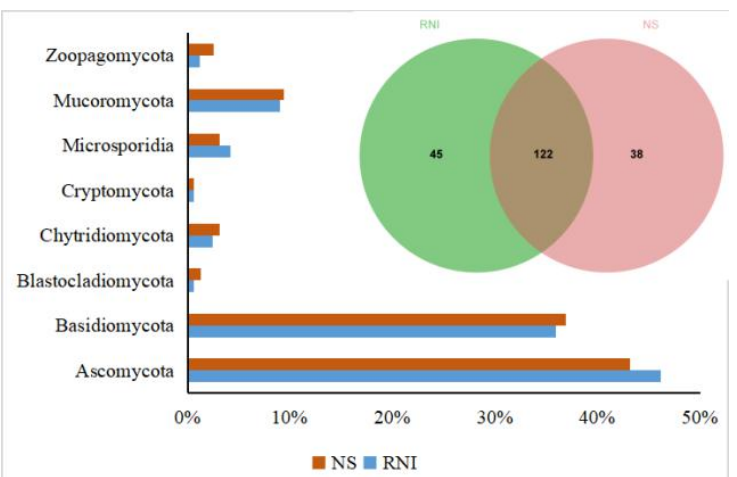

Figure S5 Relative abundances of different nodes in NS and RNI networks. (A)

Proportion of nodes belonging to different phylogenetic groups in the NS and RNI bacterial networks; Venn diagrams indicate the number of nodes shared and not shared by the NS and RNI bacterial networks. (B) Proportion of nodes belonging to different phylogenetic groups in the NS and RNI fungal networks; Venn diagrams indicate the number of nodes shared and not shared by the NS and RNI fungal networks. 
A

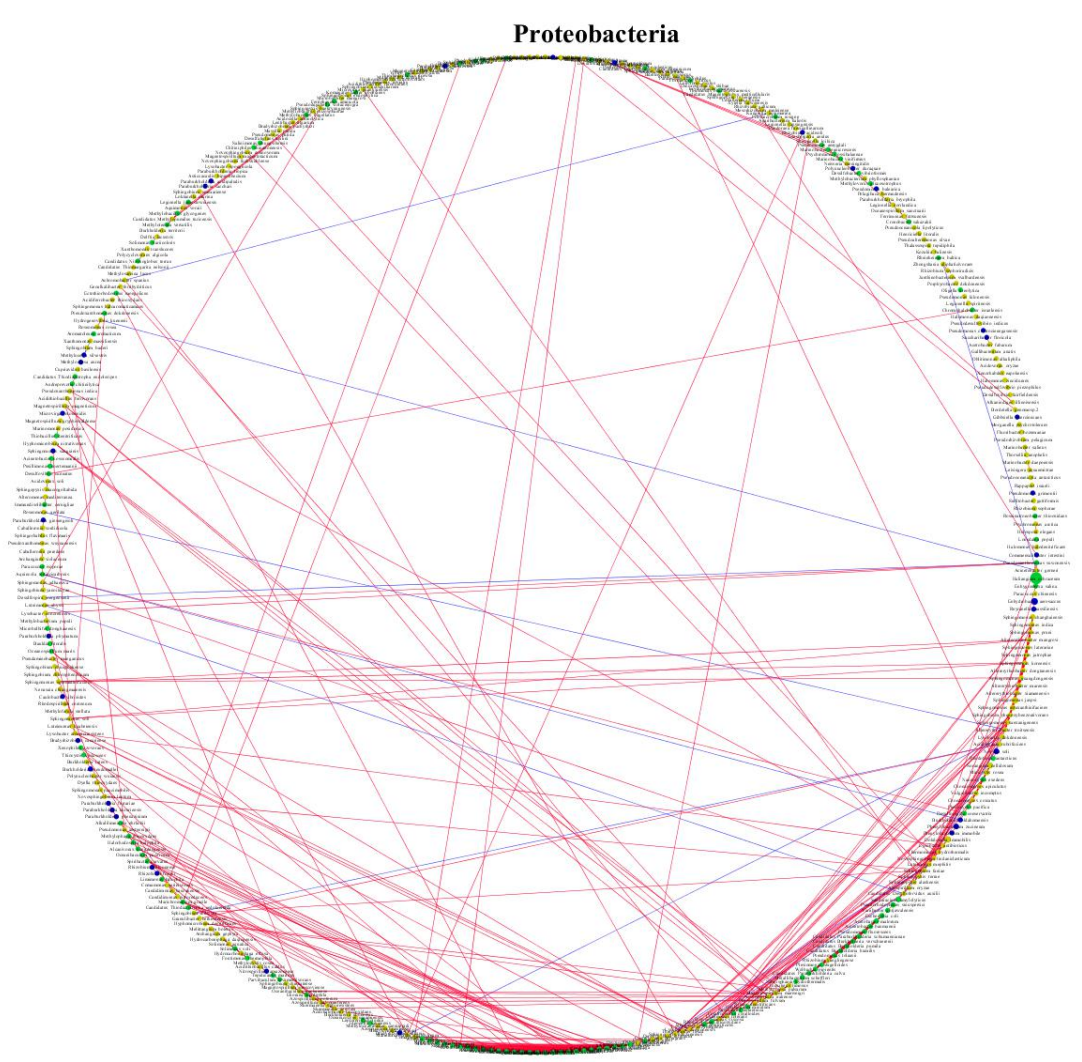

B

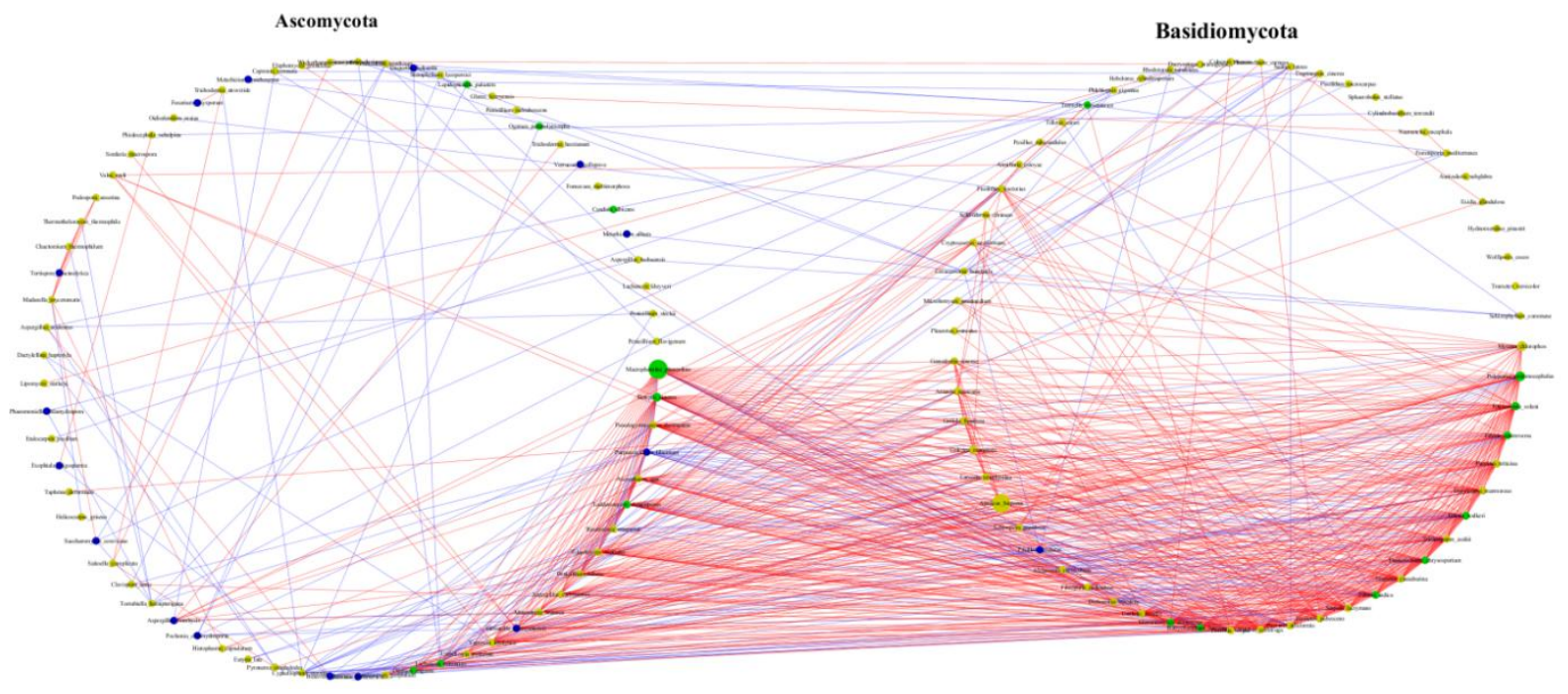

Figure S6 Sub-network of nodes belonging to Proteobacteria (A), or Ascomycota and

Basidiomycota (B). The circle sizes represent the mean read relative abundance of the differentially abundant taxa. Bacterial or fungal species that are significantly enriched in the comparison between NS and RNI are indicated in green for NS and in blue for RNI; nonsignificant taxa are indicated in yellow. Red color lines represent positive interaction. Blue color lines represent negative interaction. 


\section{A}

$$
\begin{array}{ccc}
0.0 & 2.72855 \mathrm{E}-4 \\
\text { NS } & \text { RNI }
\end{array}
$$
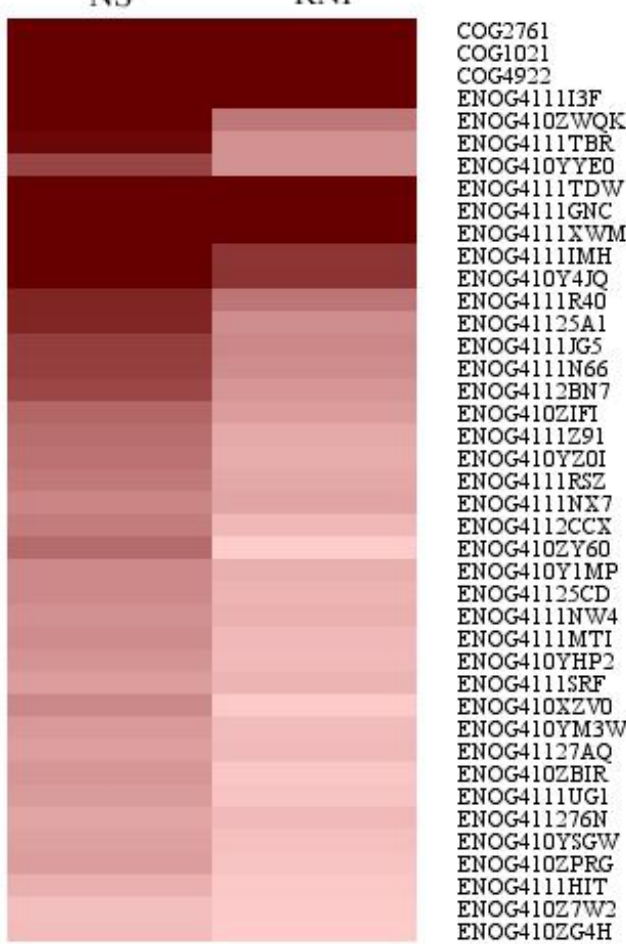

B

C

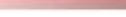

$0.0 \quad 2.06758 \mathrm{E}-5$

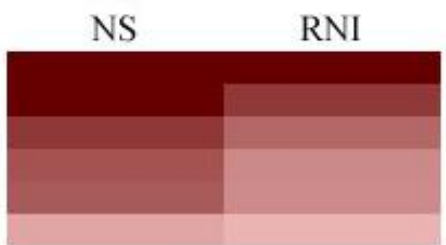

ENOG 410XQEG ENOG41119YV ENOG 41 0ZQEU ENOG 41123FU ENOG $4111 \mathrm{G} 43$ ENOG41126IS

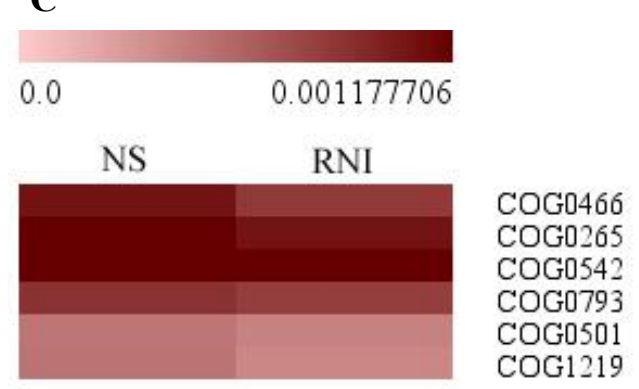

D

0.0

1. $72855 \mathrm{E}-6$
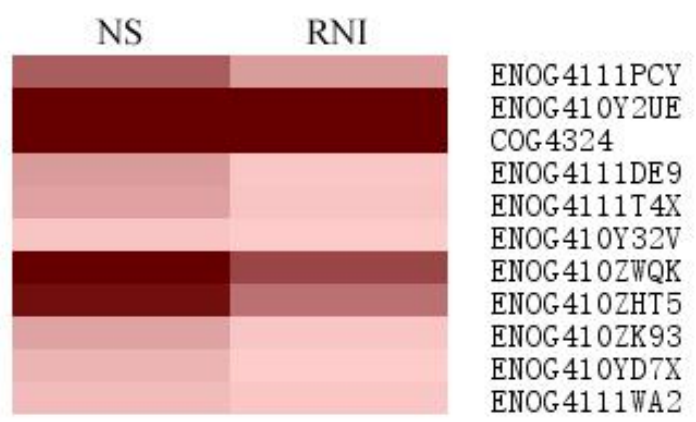

ENOG 4111PCY

COG 4324

ENOG 4111DE9

ENOG 4111T4X

ENOG 410ZHT5

ENOG $4102 \mathrm{~K} 93$

ENOG 4111WA2

Figure S7 Heatmaps depict relative abundance of the significantly enriched genes involved in polyketide biosynthesis (A), CAZymes (B) or enzymes (C, D) in the NS microbiota as compared to the RNI microbiota. 


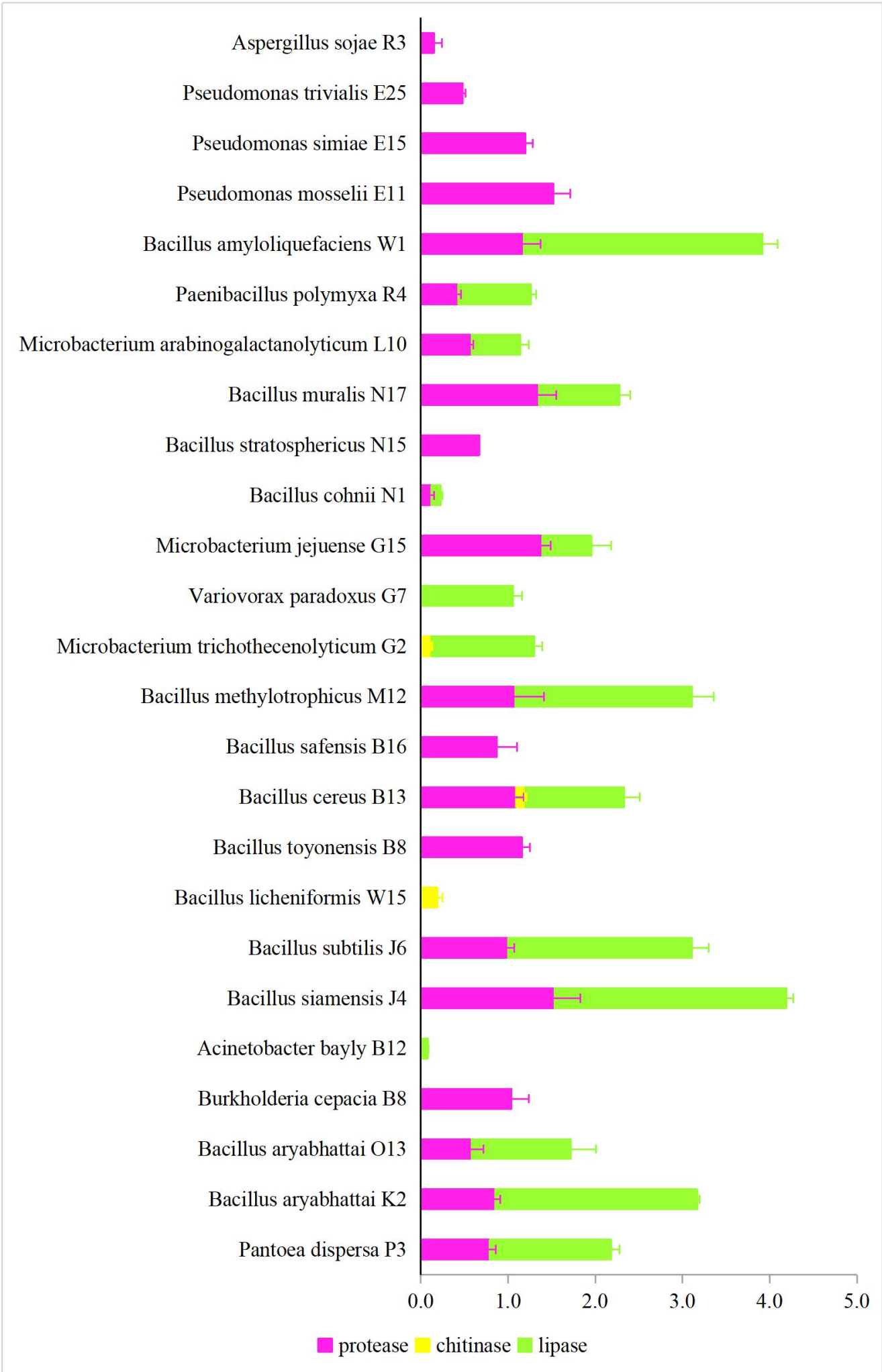

Figure S8 The protease, chitinase and lipase activities of nematicidal bacteria. The enzymic activity was represented by the ratio of clearing zone diameter to the bacterial and fungal colony diameter. 

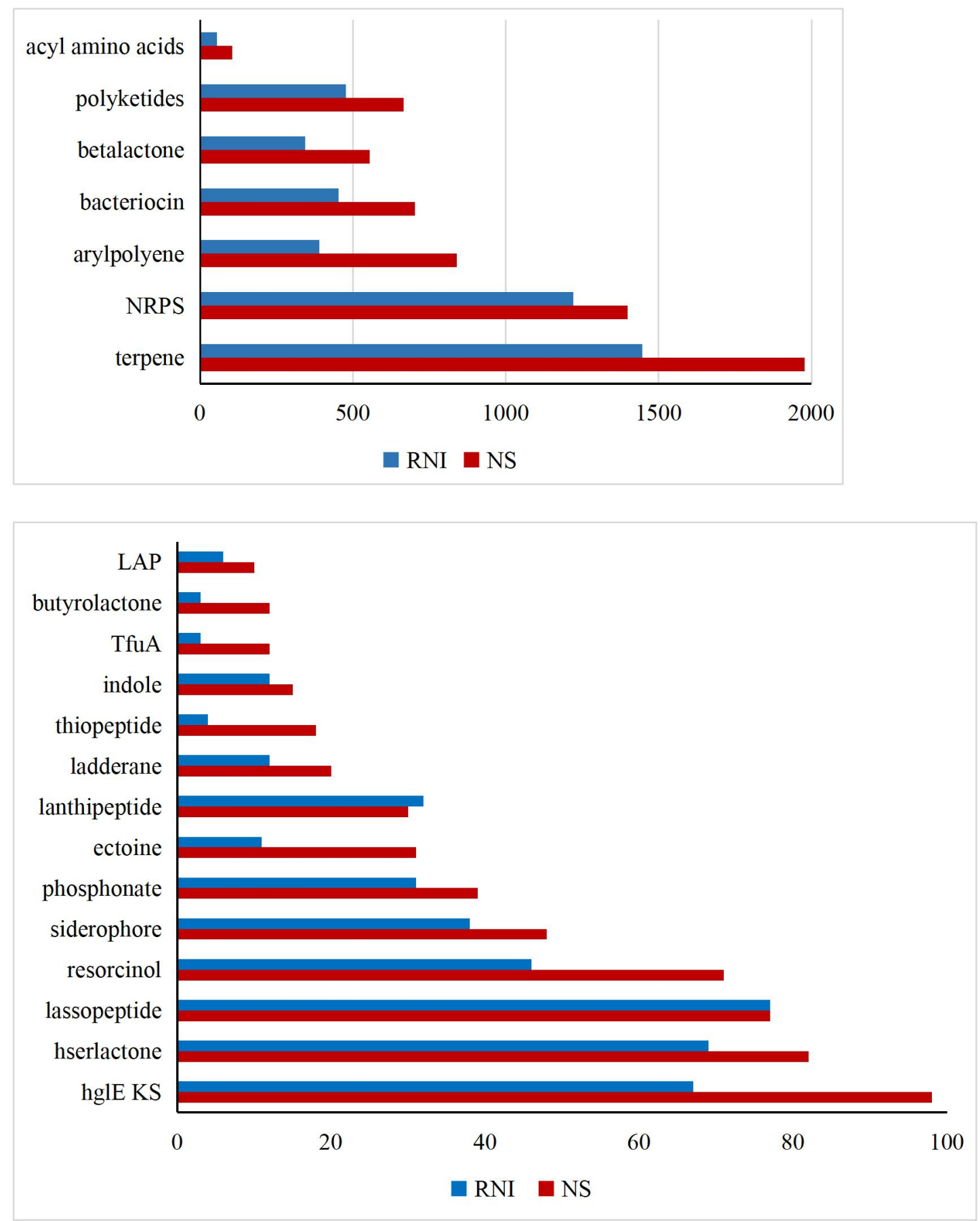

Figure S9 The abundances of BGCs in two groups. Comparing BGCs gene abundance between the NS and RNI microbiota. 


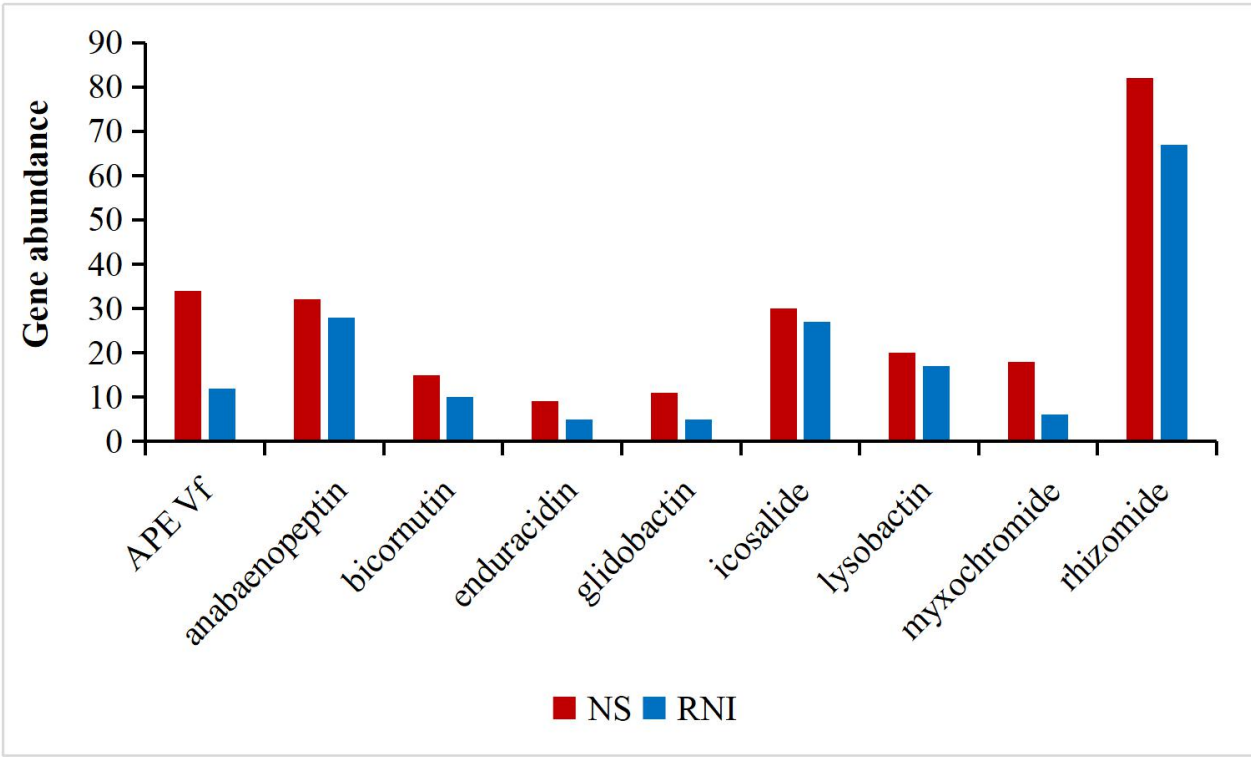

Figure S10 Comparing gene abundances between the NS and RNI BGCs network. 
$\mathbf{A}$

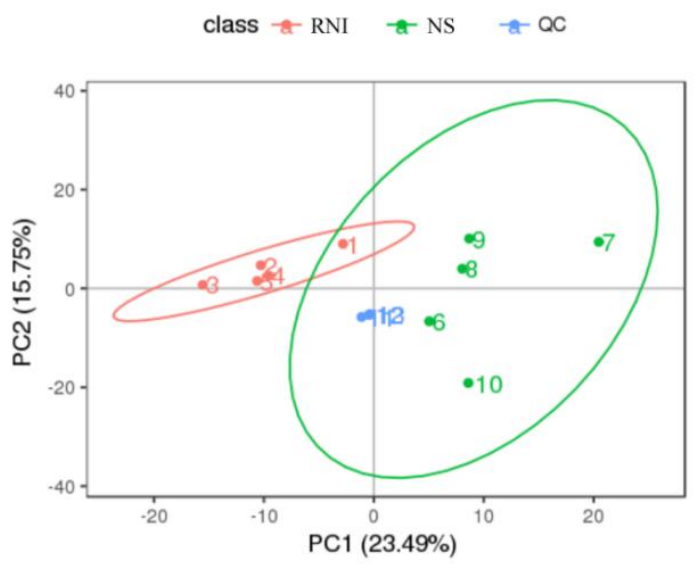

B

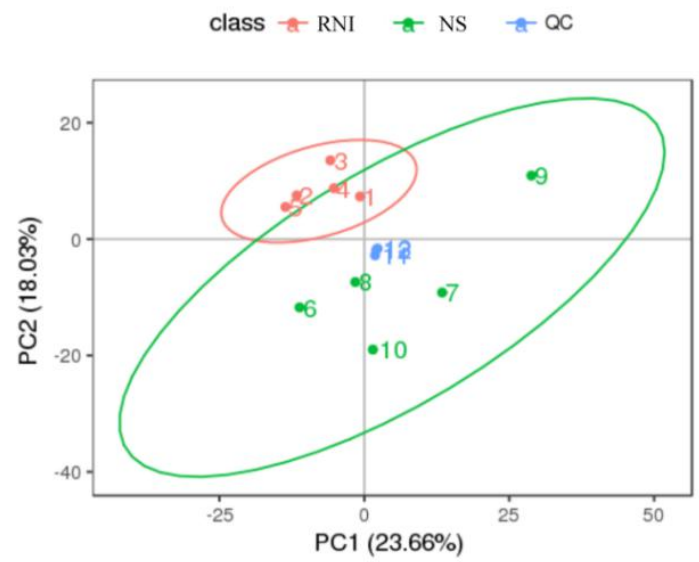

Figure S11 Metabolomics analysis of rhizosphere microbiota in the NS and RNI soils.

(A), (B): PCA analysis in negative polarity mode and positive polarity mode, respectively. 
$\mathbf{A}$

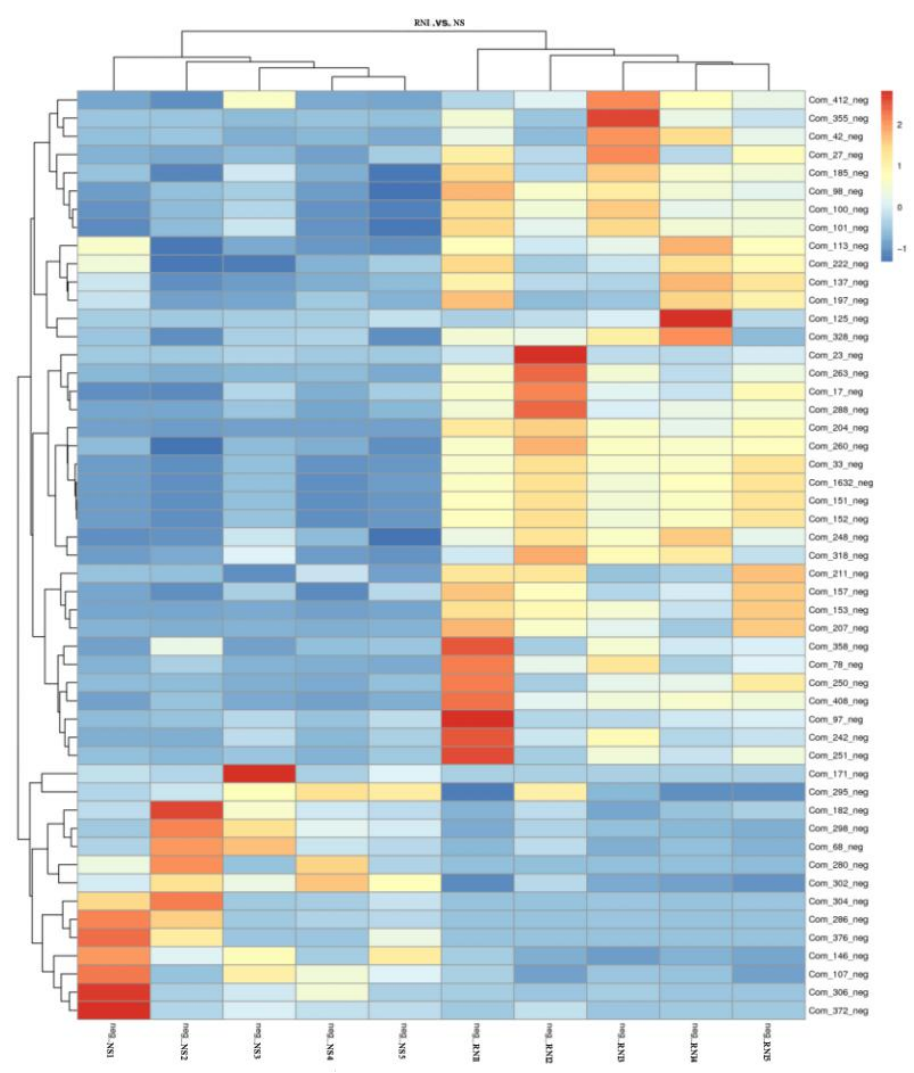

B

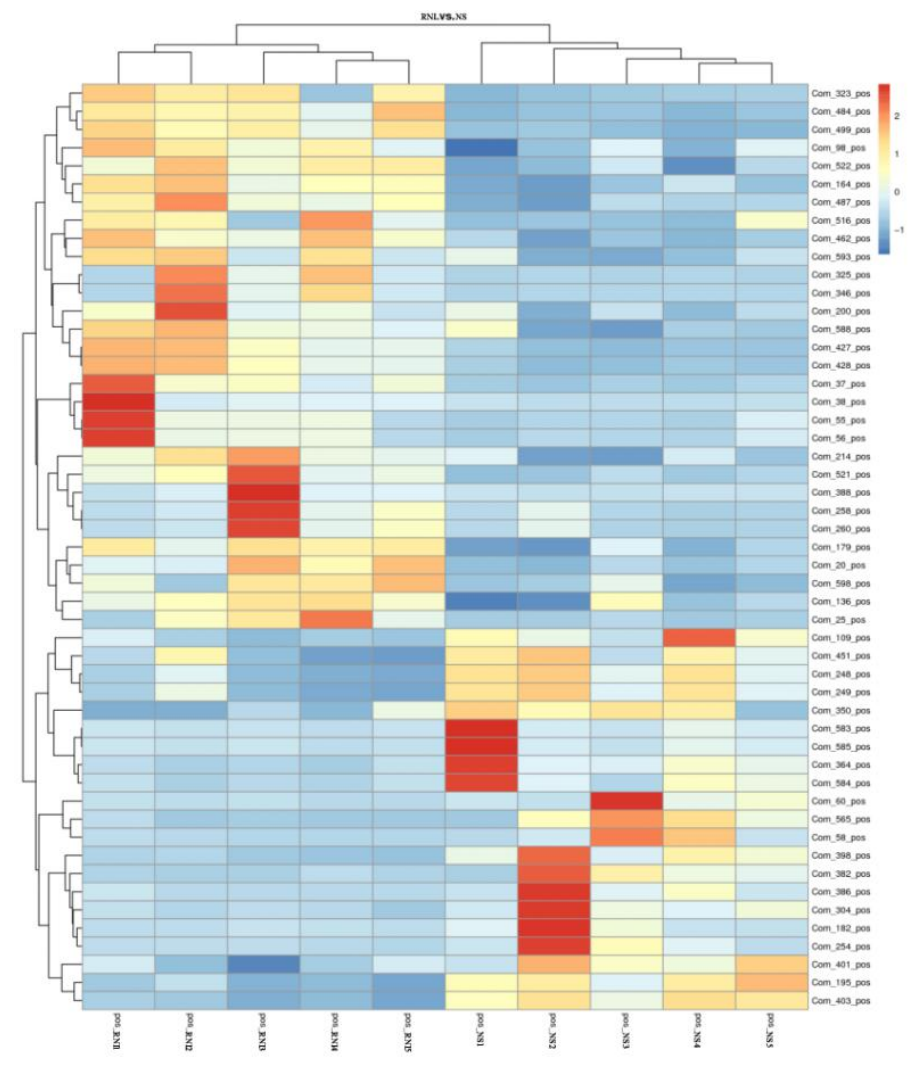

Figure S12 The hierarchical clustering of different metabolites in negative polarity mode (A) and positive polarity mode (B), respectively. 
$\mathbf{A}$

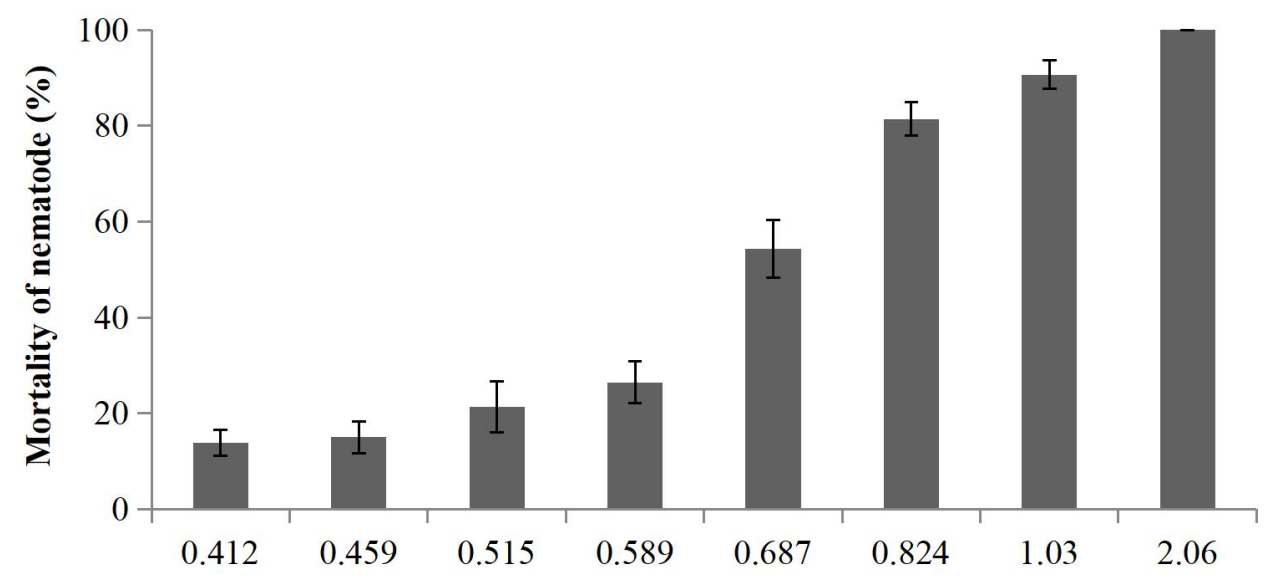

Concentration of acetophenone $(\mu \mathrm{g} / \mathrm{ml})$

B

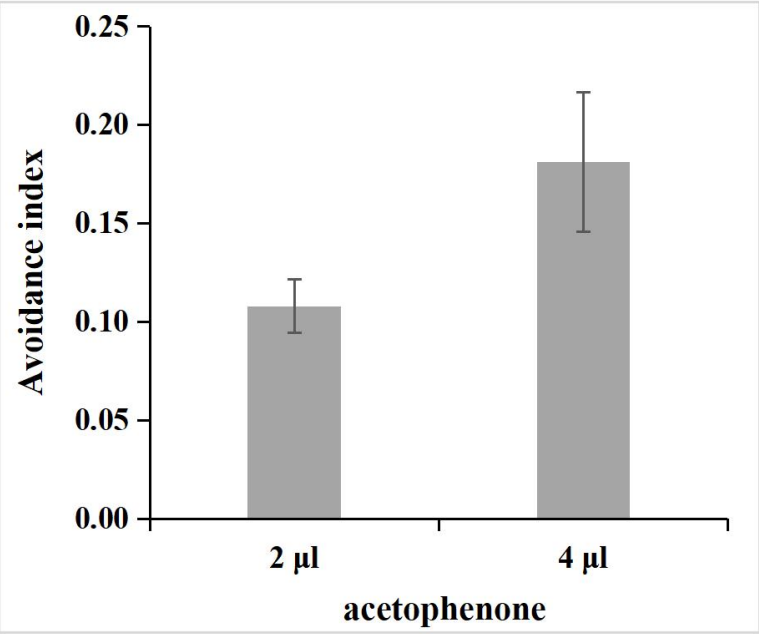

C

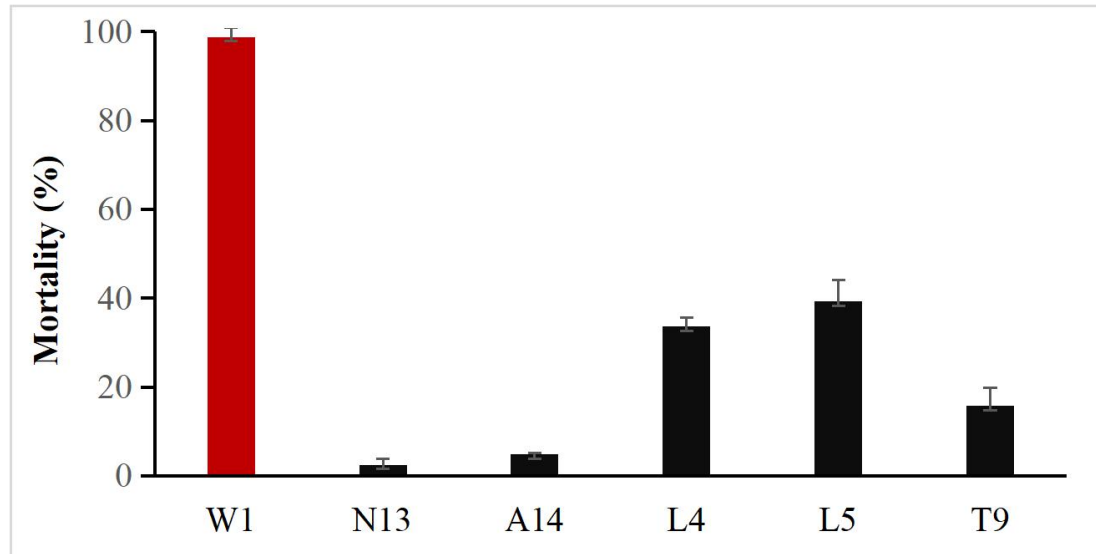


D

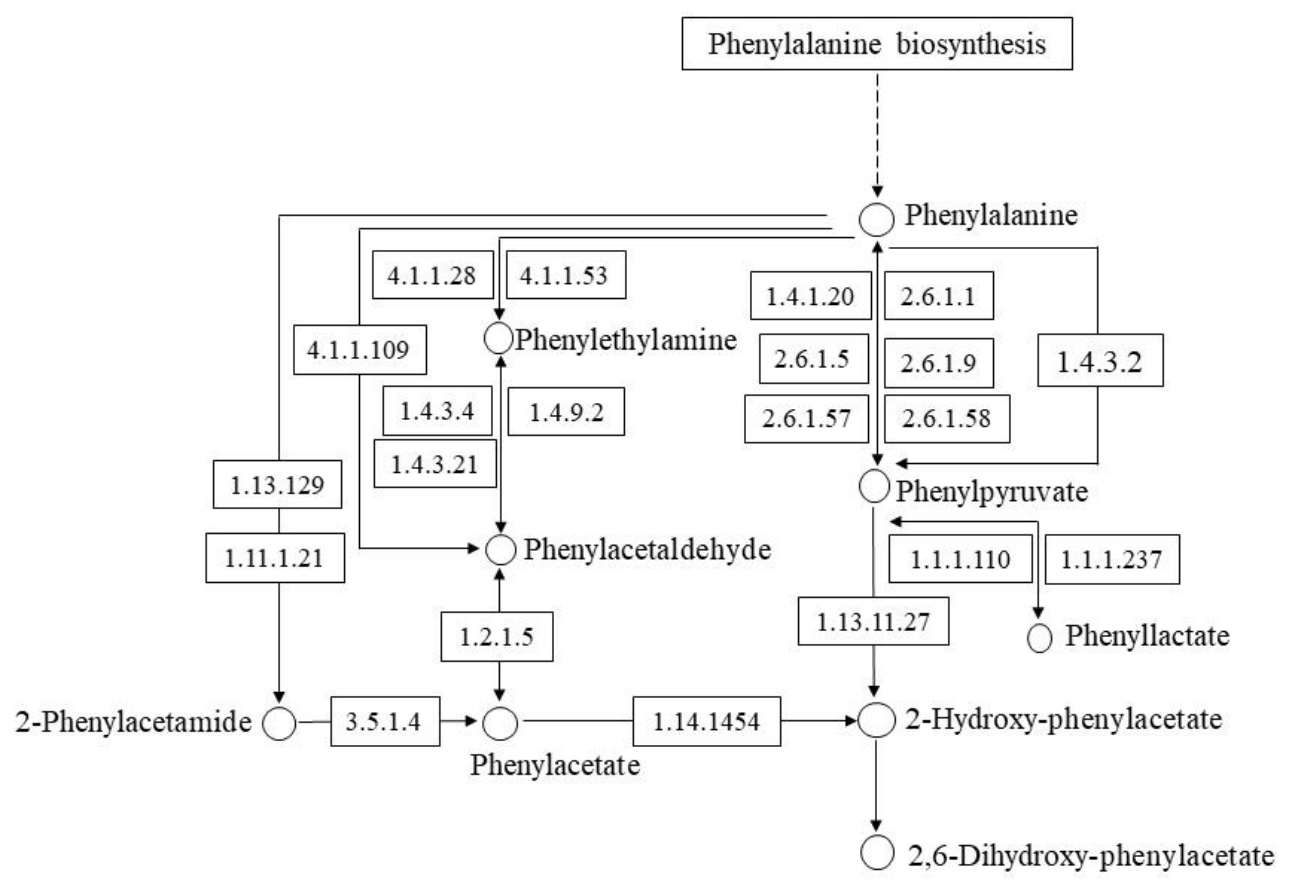

Figure S13 Nematicidal activity of metabolites and microbes. (A) Nematicidal activity of different concentrations of acetophenone against $M$. incognita. (B) Avoidance of acetophenone against M. incognita. (C) Nematicidal activity of $B$. amyloliquefaciens W1, $B$. butanolivorans N13, B. drentensis A14, B. marisflavi L4, B. wiedmannii L5, B. tequilensis T9, respectively. (D) Phenylalanine metabolism pathway from KEGG. 
Table S1 Properties of non-infected (NS) and root-knot nematode infected soils (RNI)

NS

Number of M. incognita
Urease (mg / g)
Phosphatase (mg / g)
Invertase (mg / g)
Catalase (mg / g)
pH
AK (mg / kg)
AP (mg / kg)
AN (mg / kg)
SOM (g / kg)
CEC (cmol / kg)
Fe (mg / kg)
Mn (mg / kg)
Zn (mg / kg)
Ca (mg / kg)
Mg (mg / kg)
B (mg / kg)
SWC (\%)
BD (g / cm $\left.{ }^{3}\right)$
STP (\%)
SCMC (\%)
SCP (\%)
SAP (\%)
MWD (mm)

$0.98 \pm 1.01$

$0.18 \pm 0.11 \mathrm{a}$

$1.23 \pm 0.51 \mathrm{~b}$

$5.23 \pm 3.89 \mathrm{a}$

$0.95 \pm 0.20 \mathrm{a}$

$5.17 \pm 0.43 \mathrm{a}$

$400.28 \pm 145.44 \mathrm{~b}$

$44.29 \pm 8.42 \mathrm{a}$

$121.52 \pm 33.77 \mathrm{a}$

$25.40 \pm 2.66 \mathrm{a}$

$14.70 \pm 1.95 \mathrm{a}$

$91.86 \pm 40.18 \mathrm{a}$

$57.80 \pm 25.29 \mathrm{a}$

$6.61 \pm 3.81 \mathrm{a}$

$1188.04 \pm 448.88 \mathrm{a}$

$68.82 \pm 35.09 \mathrm{a}$

$0.27 \pm 0.14 \mathrm{a}$

$13.64 \pm 1.28 \mathrm{a}$

$1.20 \pm 0.07 b$

$54.28 \pm 2.15 \mathrm{a}$

$15.83 \pm 1.66 \mathrm{a}$

$18.97 \pm 1.56 \mathrm{a}$

$35.31 \pm 2.38 \mathrm{a}$

$1.27 \pm 0.41 \mathrm{a}$

\section{RNI}

$$
\begin{gathered}
1091.76 \pm 833.87 \mathrm{a} \\
0.13 \pm 0.04 \mathrm{a} \\
1.95 \pm 1.05 \mathrm{a} \\
2.61 \pm 1.07 \mathrm{~b} \\
0.77 \pm 0.14 \mathrm{~b} \\
4.86 \pm 0.19 \mathrm{~b} \\
483.48 \pm 54.21 \mathrm{a} \\
54.89 \pm 19.66 \mathrm{a} \\
117.39 \pm 8.73 \mathrm{a} \\
25.03 \pm 2.25 \mathrm{a} \\
12.75 \pm 0.57 \mathrm{~b} \\
0.93 \pm 0.36 \mathrm{~b} \\
42.98 \pm 18.98 \mathrm{a} \\
5.05 \pm 1.82 \mathrm{a} \\
687.82 \pm 236.91 \mathrm{~b} \\
51.83 \pm 20.87 \mathrm{a} \\
0.25 \pm 0.06 \mathrm{a} \\
12.68 \pm 0.77 \mathrm{~b} \\
1.25 \pm 0.03 \mathrm{a} \\
52.75 \pm 1.05 \mathrm{~b} \\
14.54 \pm 1.01 \mathrm{~b} \\
18.12 \pm 1.21 \mathrm{a} \\
34.63 \pm 1.63 \mathrm{a} \\
0.80 \pm 0.18 \mathrm{~b}
\end{gathered}
$$

Table S2 Correlation of soil properties and number of Meloidogyne incognita

\begin{tabular}{lrr}
\multicolumn{1}{r}{ Soil properties } & \multicolumn{1}{l}{ r } \\
Urease & -0.279 & 0.136 \\
Phosphatase & 0.074 & 0.699 \\
Invertase & -0.338 & 0.067 \\
Catalase & $\mathbf{- 0 . 3 8 3}$ & $\mathbf{0 . 0 3 7}$ \\
pH & -0.29 & 0.119 \\
AK & 0.326 & 0.079 \\
AP & $\mathbf{0 . 3 9 1}$ & $\mathbf{0 . 0 3 3}$ \\
AN & -0.03 & 0.874 \\
SOM & -0.005 & 0.981 \\
CEC & $\mathbf{- 0 . 3 9 6}$ & $\mathbf{0 . 0 3}$ \\
Fe & $\mathbf{- 0 . 5 9 2}$ & $\mathbf{0 . 0 0 1}$ \\
Mn & -0.279 & 0.135 \\
Zn & -0.208 & 0.27
\end{tabular}




$\begin{array}{lrr}\text { Ca } & \mathbf{- 0 . 4 3 7} & \mathbf{0 . 0 1 6} \\ \text { Mg } & -0.03 & 0.874 \\ \text { B } & -0.047 & 0.805 \\ \text { SWC } & -0.297 & 0.111 \\ \text { BD } & \mathbf{0 . 3 6 5} & \mathbf{0 . 0 4 7} \\ \text { STP } & \mathbf{- 0 . 3 6 3} & \mathbf{0 . 0 4 9} \\ \text { SCMC } & -0.304 & 0.103 \\ \text { SCP } & -0.172 & 0.364 \\ \text { SAP } & -0.207 & 0.273 \\ \text { MWD } & \mathbf{- 0 . 4 3 2} & \mathbf{0 . 0 1 7}\end{array}$

Table S3 Metagenome sequence statistics after filtering

\section{Data Clean}

Total Raw Data

4,211,442 Mbp

Average Raw Data

$135,852.97 \mathrm{Mbp}$

Assembly and Mix-Assembly

Scaffolds (Average)

344610

Total length (nt)

Average length (nt)

$9,998,862,533 \mathrm{bp}$

Longest length (nt)

$935.97 \mathrm{bp}$

$284,861 \mathrm{bp}$

N50 length (nt)

893.84 bp

N90 length (nt)

$542.84 \mathrm{bp}$

Scaftigs (Average)

344610

Total length (nt)

Average length (nt)

$9,998,862,533 \mathrm{bp}$

$936 \mathrm{bp}$

N50 length (nt)

$894 \mathrm{bp}$

N90 length (nt)

$543 \mathrm{bp}$

Gene Prediction

Total ORFs

16725240

Average ORFs

539524

Gene catalogue

7677442

Complete ORFs

$1,812,302(23.61 \%)$

Total length (Mbp)

4105.43

Average length (bp)

534.74

GC percent

$63.42 \%$

Taxonomic Annotation

Gene catalogue

7677442

Annotated on NR

Annotated on Unclassified

$5,678,146(73.96 \%)$

Annotated on Kingdom level

$34.95 \%$

$65.05 \%$

Annotated on Phylum level

$57.52 \%$

Annotated on Class level

$52.52 \%$ 
Annotated on Order level

Annotated on Family level

Annotated on Genus level

Annotated on Species level

Assigned Phyla(top 5)

Sign_diff Phyla(top 5)

Functional Annotation

Gene catalogue

Annotated on KEGG

Annotated on $\mathrm{KO}$

Annotated on EC

Annotated on pathway

Annotated on eggNOG

Annotated on OG

Annotated on CAZy

CARD Annotation

Gene catalogue

Annotated on CARD

Annotated AROs
$48.42 \%$

$43.30 \%$

$39.22 \%$

$29.79 \%$

Proteobacteria

k_ Bacterial;p_Proteobacteria

$$
\begin{gathered}
7677442 \\
4,698,328(61.20 \%) \\
2,616,196(34.08 \%) / 8,019 \\
1,660,255(21.63 \%) / 2,760 \\
1,687,236(21.98 \%) / 405 \\
4,442,108(57.86 \%) \\
4,442,108(57.86 \%) / 45,236 \\
255,379(3.33 \%)
\end{gathered}
$$

7677442

1722

735

Table S4 CDS statistics of each sample

$\begin{array}{lcccc}\text { Treatment } & \text { Sample ID } & \text { ORFs NO. } & \text { Total Len. (Mbp) } & \text { Average Len. (bp) } \\ \text { Non-infected soils } & \text { NS1 } & 437,836 & 226.38 & 517.04 \\ \text { NS2 } & 390,789 & 203.19 & 519.96 \\ \text { NS3 } & 759,756 & 407.73 & 536.66 \\ \text { NS4 } & 509,777 & 259.85 & 509.72 \\ \text { NS5 } & 312,173 & 152.04 & 487.04 \\ \text { NS6 } & 579,246 & 302.07 & 521.49 \\ \text { NS7 } & 392159 & 202.63 & 516.7 \\ \text { NS8 } & 777797 & 429.97 & 552.8 \\ \text { NS9 } & 483135 & 255.6 & 529.04 \\ \text { NS10 } & 236114 & 115.98 & 491.19 \\ \text { NS11 } & 578,225 & 313.07 & 541.43 \\ \text { RS12 } & 517,214 & 271.54 & 525.01 \\ \text { infected soils } & 784483 & 418.17 & 533.05 \\ & \text { NS13 } & 404746 & 196.39 & 485.23 \\ \text { NS14 } & \text { NS15 } & 151614 & 70.72 & 466.43 \\ & \text { RNI1 } & 742392 & 402.52 & 542.2 \\ & \text { RNI2 } & 564414 & 296.43 & 561.63 \\ & \text { RN14 } & 704704 & 395.78 & 549.23\end{array}$




$\begin{array}{cccc}\text { RNI5 } & 946039 & 528.48 & 558.62 \\ \text { RNI6 } & 582316 & 311.18 & 534.39 \\ \text { RNI7 } & 534738 & 276.24 & 516.59 \\ \text { RNI8 } & 288109 & 144.52 & 501.61 \\ \text { RNI9 } & 539190 & 301.05 & 558.34 \\ \text { RNI10 } & 570671 & 301.03 & 527.5 \\ \text { RNI11 } & 517614 & 272.63 & 526.7 \\ \text { RNI12 } & 511731 & 266.75 & 521.26 \\ \text { RNI13 } & 604914 & 319.65 & 528.42 \\ \text { RNI14 } & 547648 & 285.91 & 522.06 \\ \text { RNI15 } & 331647 & 163.74 & 493.73\end{array}$

Table S5 Correlations and topological properties of the co-occurrence fungal network analysis

$\begin{array}{lcc}\text { Network properties } & \text { NS } & \text { RNI } \\ \text { Number of nodes } & 160 & 167 \\ \text { Number of edges } & 1251 & 581 \\ \text { Positive edges } & 905 & 522 \\ \text { Negative edges } & 345 & 58 \\ \text { Modularity } & 0.242 & 0.443 \\ \text { Number of module } & 6 & 13 \\ \text { Average degree (avgK) } & 15.637 & 6.958 \\ \text { Average clustering coefficient (avgCC) } & 0.374 & 0.239 \\ \text { Average path distance (GD) } & 2.879 & 4.376\end{array}$

Table S6 Correlations and topological properties of the co-occurrence bacterial network analysis

$\begin{array}{lcc}\text { Network properties } & \text { NS } & \text { RNI } \\ \text { Number of nodes } & 1121 & 1082 \\ \text { Number of edges } & 2947 & 2465 \\ \text { Positive edges } & 2737 & 2212 \\ \text { Negative edges } & 209 & 252 \\ \text { Modularity } & 0.744 & 0.786 \\ \text { Number of module } & 199 & 175 \\ \text { Average degree (avgK) } & 5.258 & 4.556 \\ \text { Average clustering coefficient (avgCC) } & 0.232 & 0.189 \\ \text { Average path distance (GD) } & 6.908 & 8.004\end{array}$


Table S7 Correlations and topological properties of the co-occurrence functional

\section{network analysis}

\begin{tabular}{lcc}
\multicolumn{1}{c}{ Network properties } & NS & RNI \\
Number of nodes & 631 & 693 \\
Number of edges & 5720 & 8450 \\
Positive edges & 3488 & 5166 \\
Negative edges & 2231 & 3283 \\
Modularity & 0.520 & 0.307 \\
Number of module & 40 & 35 \\
Average degree (avgK) & 18.130 & 24.387 \\
Average clustering coefficient (avgCC) & 0.333 & 0.296 \\
Average path distance (GD) & 5.806 & 6.035
\end{tabular}

Table S8 Correlation of metabolites and number of Meloidogyne incognita

Name of metabolites

2-Methyl-4,6-dinitrophenol

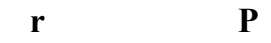

Dodecanedioic acid

$-.725 *$

0.018

$-.688^{*}$

0.028

2-hydroxymyristic acid

$-.649 *$

0.042

1-\{[(2-Aminoethoxy)(hydroxy)phosphoryl] oxy $\}$-3-hydroxy-2-propanyl stearate

11-Deoxycortisol

$-.633 * \quad 0.05$

Indole-3-acetic acid

2-Methylbenzoic acid

10-Hydroxydecanoic acid

2-Naphthalenesulfonic acid

8-Amino-7-oxononanoic acid

Acetyl-N-formyl-5-methoxykynurenamine

6-Acetylmorphine

$-.840 * * \quad 0.002$

$-.640 * \quad 0.046$

$-.657 * \quad 0.039$

$-.822 * * \quad 0.003$

$-.655 * \quad 0.040$

$-.824 * * \quad 0.003$

-.664* $\quad 0.036$

$-.638 * \quad 0.047$

$-.820 * * \quad 0.004$

$-.736 * \quad 0.015$

$-.637 * \quad 0.048$

$-.754 * \quad 0.012$

$-.806 * * \quad 0.005$

$-.634 * \quad 0.049$

-.665* 0.036

$-.736^{*} \quad 0.015$

$-.764 * \quad 0.010$ 


\section{Table S9 Metabolites in Bacillus amyloliquefaciens W1 culture}

\begin{tabular}{llcccc} 
Compound_ID & \multicolumn{1}{c}{ Name } & Formula & $\begin{array}{c}\text { Molecular } \\
\text { Weight }\end{array}$ & $\begin{array}{c}\text { RT } \\
{[\mathbf{m i n}]}\end{array}$ pos_W1_3 pos_W1_1 pos_W1_2 \\
Com_451_pos & Acetophenone & C8 H8 O & 120.05758 & 10.856474693759 .4313937571 .3 & 328412584.6 \\
Com_5576_pos & 2-Phenylacetamide & C8 H9 N O & 135.06847 & 12.69113095040 .2813217807 .26 & 11961931.05 \\
Com_464_neg & 2-Hydroxyphenylacetic acid C8 H15 N O3 & 173.10524 & 6.714146706054 .8239591727 .2 & 136310953.2 \\
Com_1091_neg Phenylpyruvic Acid & C9 H8 O3 & 164.04714 & 0.71460217768 .5969545905 .49 & 60637613.08 \\
Com_831_neg & Phenylacetaldehyde & C12 H24 O3 & 216.17237 & 11.15924876656 .02 & 10024273285858537.83
\end{tabular}




\section{Figures}

(A)
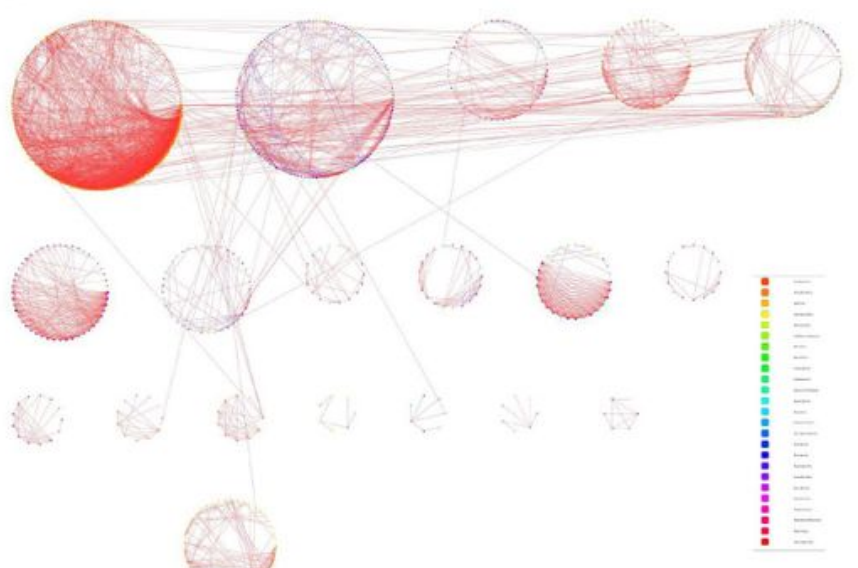

(B)

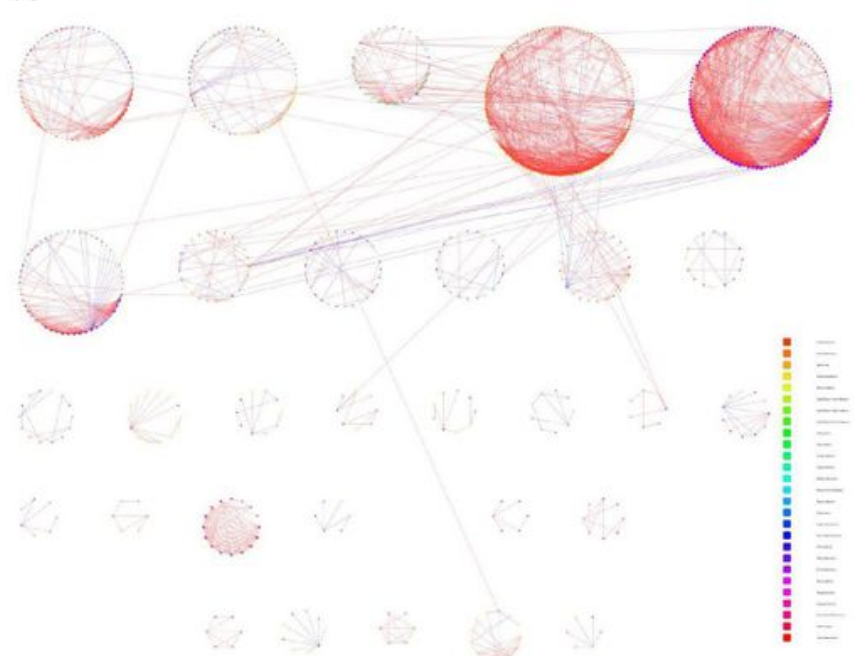

(C)

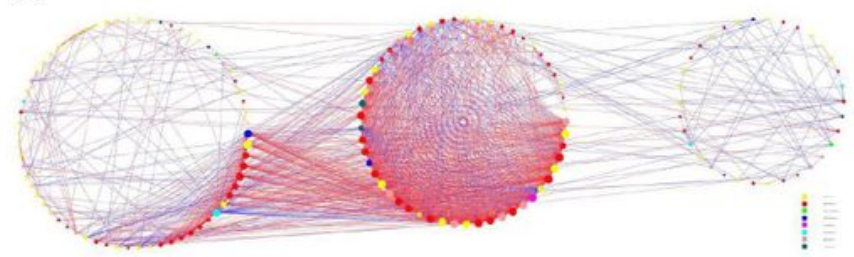

(D)

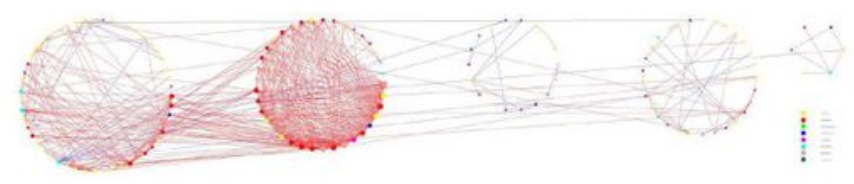

Figure 1

Taxonomic network inference of the rhizosphere microbiota. (A) and (B): Co-occurrence network based on correlation of 16S rRNA reads (species level) extracted from metagenomes of rhizosphere soil in the two conditions: non-infected soil (NS), and root-knot infected soil (RNI), respectively. (C) and (D): Co- 
occurrence network based on correlation of 18S rRNA reads (species level) extracted from metagenomes of the NS and RNI rhizosphere microbiota, respectively. The size of each node is proportional to the number of connections (node degree). Nodes belonging to different phylum are differently colored. Blue lines and red lines indicate negative and positive interactions between different nodes, respectively. Modules with more than five nodes are included.

(A)

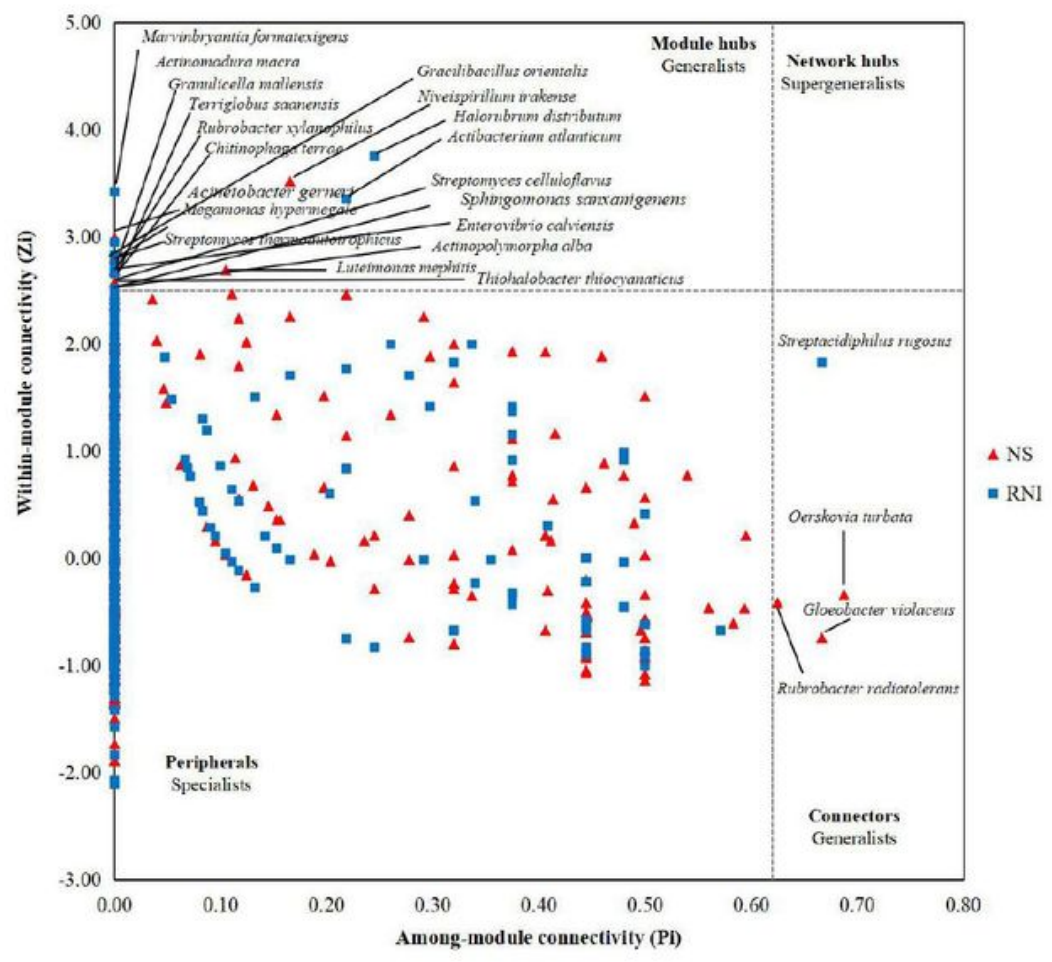

(B)

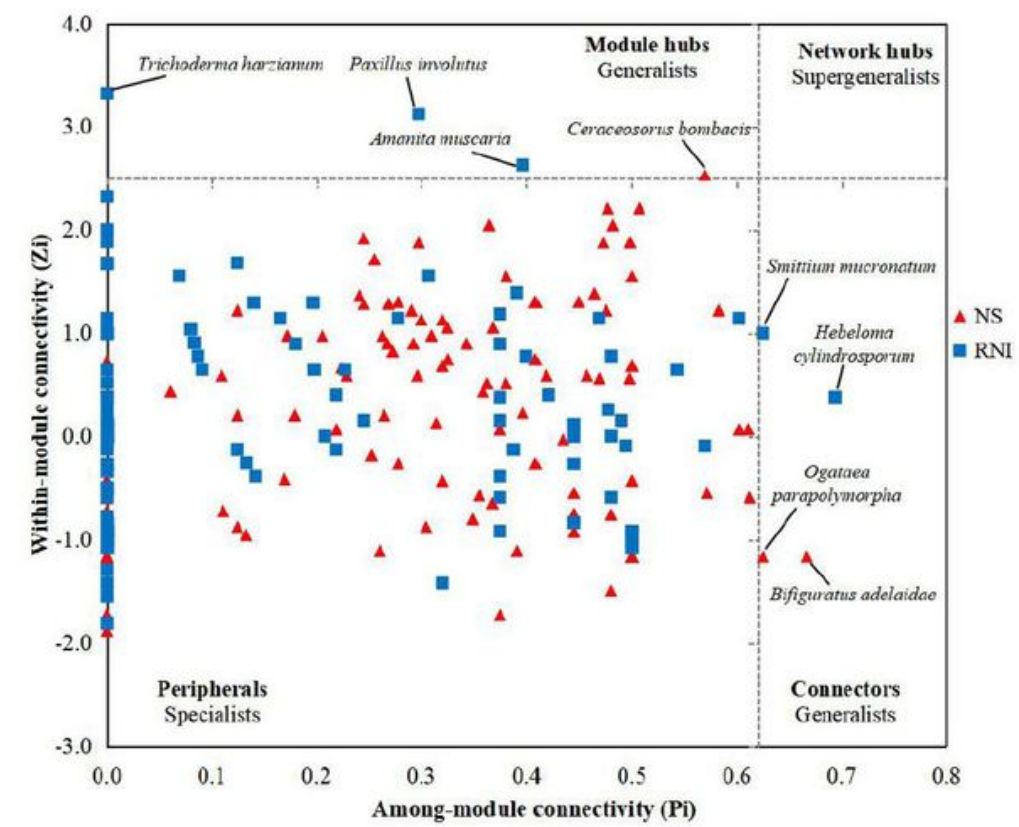

Figure 2 
$\mathrm{Zi}-\mathrm{Pi}$ plot indicates the distribution of nodes based on their topological roles in bacterial network $(\mathrm{A})$ and fungal network (B). Each symbol represents a node in the non-infected soil (NS) network (red triangle) or root-knot infected soil (RNI) network (blue square). The threshold values of Zi and Pi for categorizing nodes are 2.5 and 0.62 , respectively.
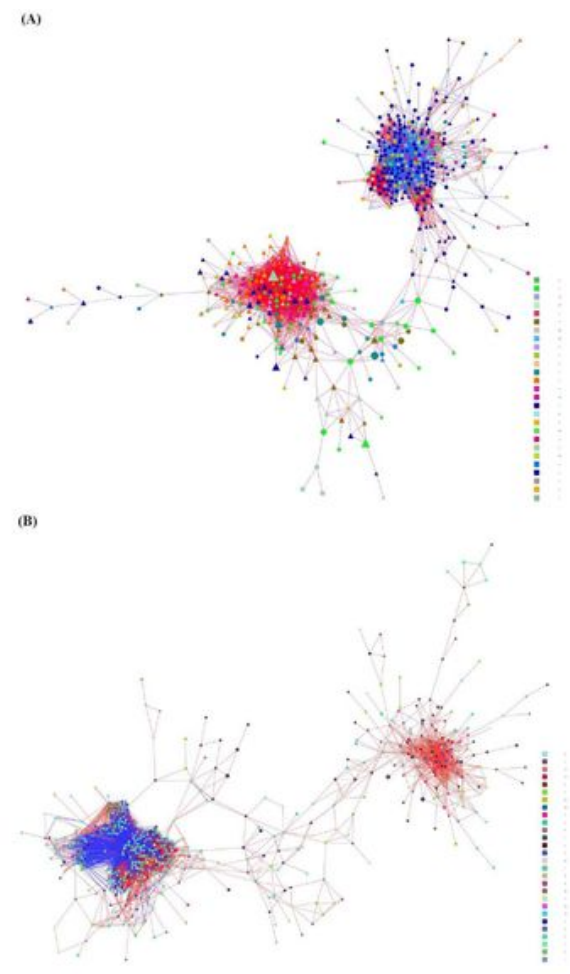

(C)

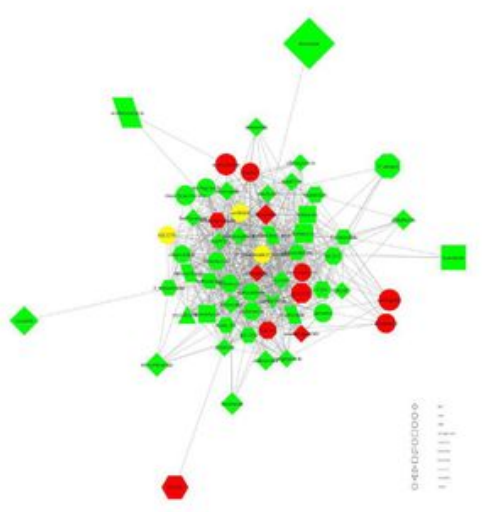

(D)

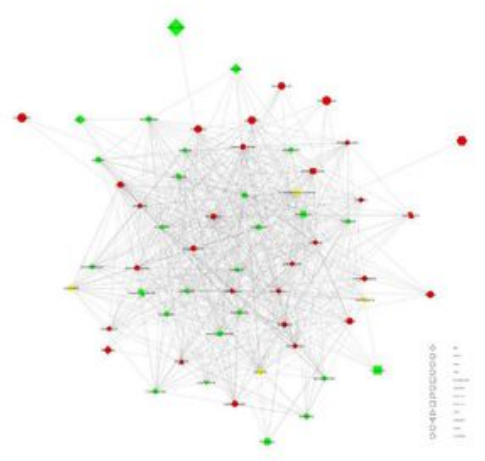

Figure 3 
Function network inference of the rhizosphere microbiota. (A) and (B): Co-occurrence network based on correlation of COG genes in the two conditions: non-infected soils (NS), and root-knot infected soils (RNI), respectively. The size of each node is proportional to the abundance of nodes. Nodes belonging to different category are differently colored. Blue lines and red lines indicate negative and positive interactions between different nodes, respectively. Node shapes are based on change of node abundance: circle, non-change nodes in any treatment; triangle, overrepresented in the NS condition; diamond, overrepresented in the RNI condition. (C) and (D): Co-occurrence network based on correlation of BGCs genes in the NS and RNI microbiota. Nodes are shaped by BGC types and the size is correlated with the abundance of each node. Node color is based on change of node abundance. Yellow, non-change nodes in any treatment; red: enriched in the RNI microbiota, green: enriched in the NS microbiota. 
(A)

(B)

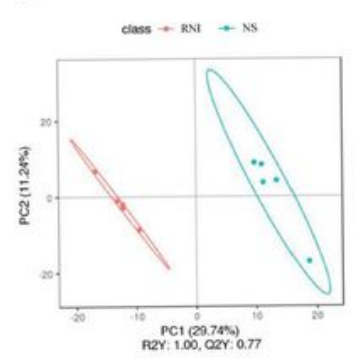

Cons $\rightarrow \mathrm{RN}+\mathrm{NS}$

(C)

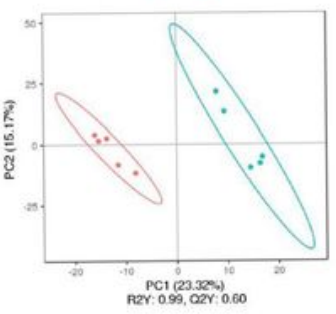

(D)

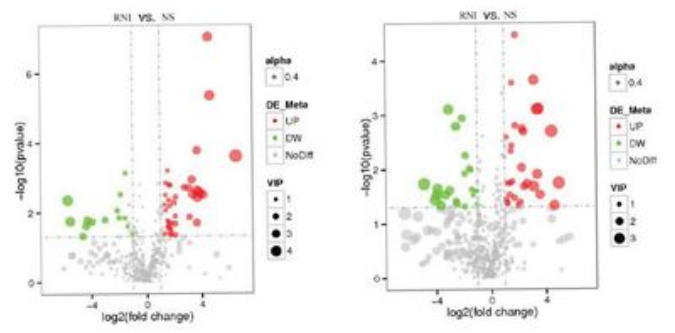

(E)

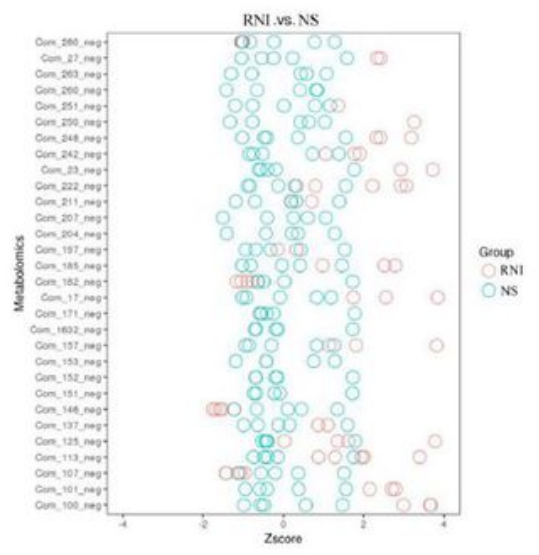

(F)

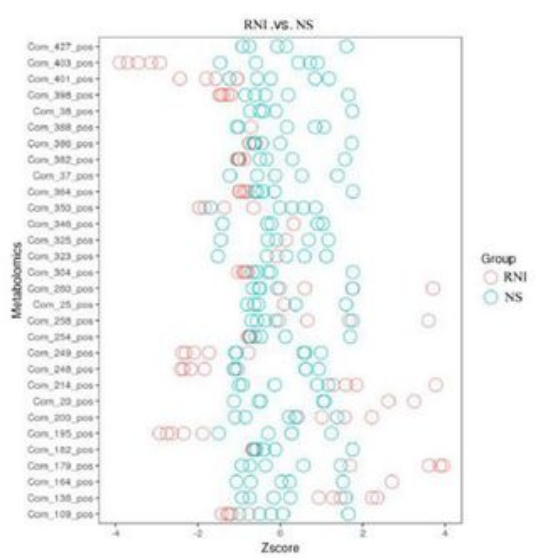

\section{Figure 4}

Metabolomics analysis of microbiota in the NS and RNI rhizosphere soils. (A) and (B): PLS-DA analysis in negative polarity mode and positive polarity mode, respectively. (C) and (D): Different metabolites in negative and positive polarity mode, respectively. $(E)$ and $(F)$ : Z-score of different metabolites in negative and positive polarity mode, respectively. 


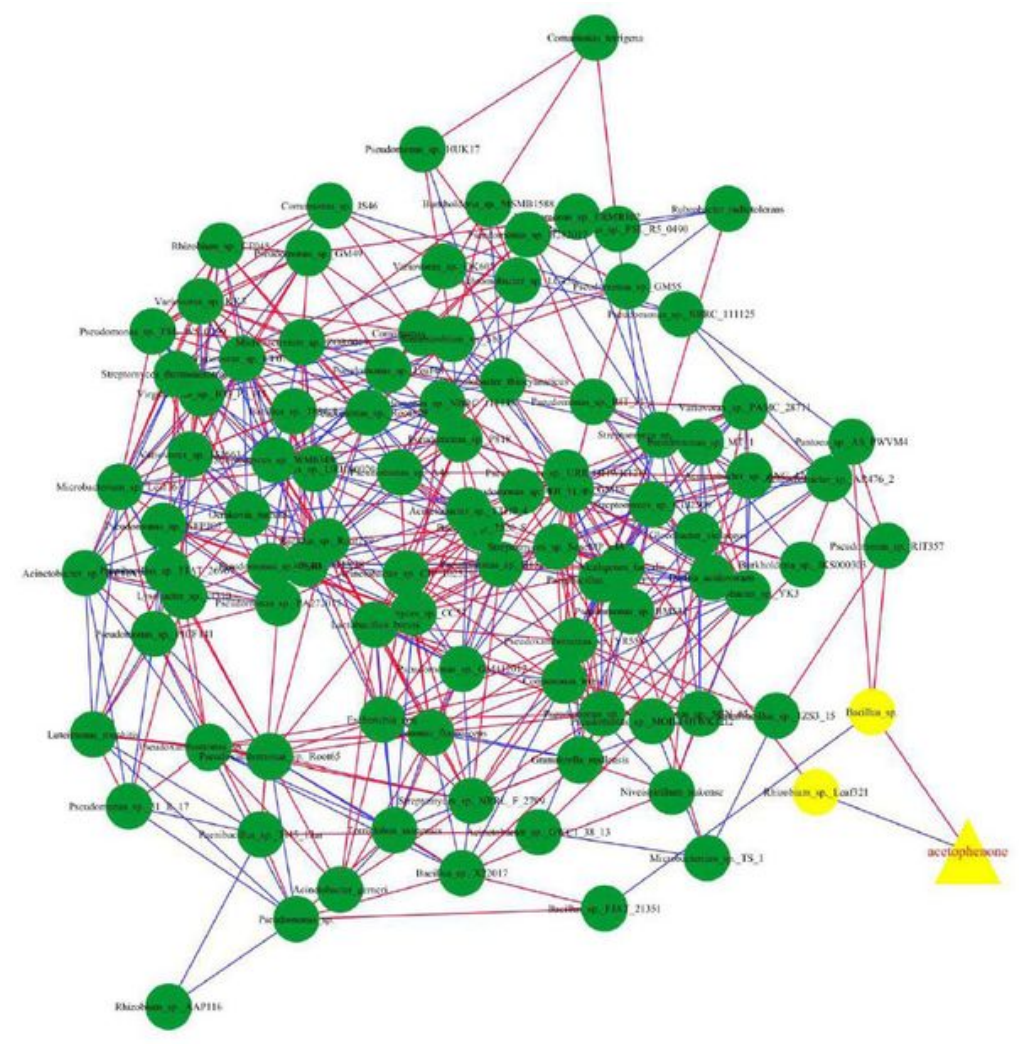

A

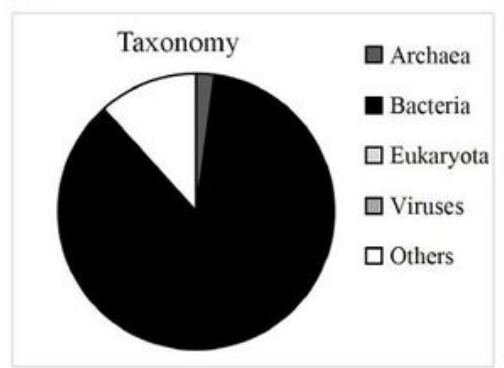

C

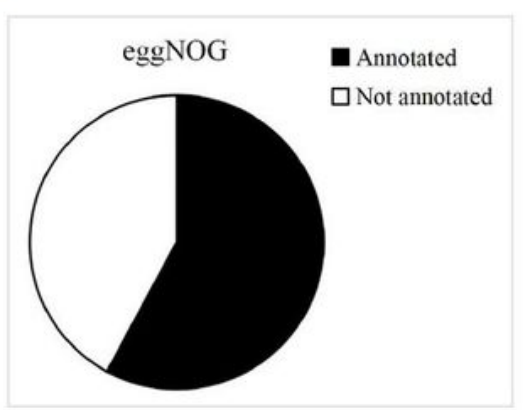

B

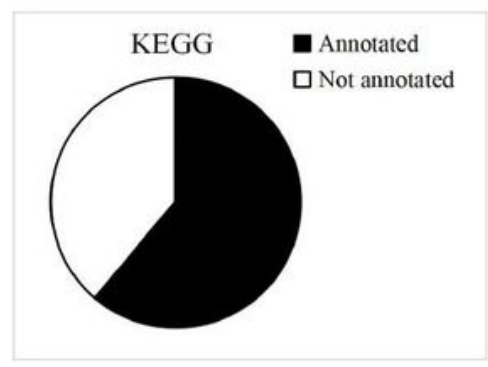

